PUBLICATIONS

NATL INST. OF STAND \& TECH

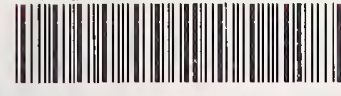

A 1.1. 107 259804

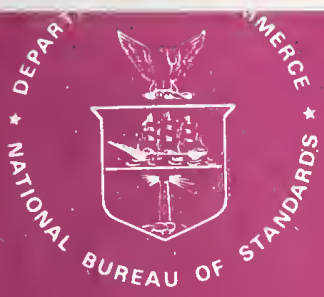

NBS SPECIAL PUBLICATION 687

U.S. DEPARTMENT OF COMMERCE/National Bureau of Standards

\title{
1984 NVLLẠ Directory of Accredited Laboratories
}

$Q C$

100

.457

No. 687

1985

C. 2 
he National Bureau of Standards' was established by an act of Congress on March 3, 1901. The

Bureau's overall goal is to strengthen and advance the nation's science and technology and facilitate

their effective application for public benefit. To this end, the Bureau conducts research and provides: (1) a basis for the nation's physical measurement system, (2) scientific and technological services for industry and government, (3) a technical basis for equity in trade, and (4) technical services to promote public safety. The Bureau's technical work is performed by the National Measurement Laboratory, the National Engineering Laboratory, the Institute for Computer Sciences and Technology, and the Center for Materials Science.

\section{The National Measurement Laboratory}

Provides the national system of physical and chemical measurement; coordinates the system with measurement systems of other nations and furnishes essential services leading to accurate and uniform physical and chemical measurement throughout the Nation's scientific community, industry, and commerce; provides advisory and research services to other Government agencies; conducts physical and chemical research; develops, produces, and distributes Standard Reference Materials; and provides calibration services. The Laboratory consists of the following centers:
- Basic Standards ${ }^{2}$

- Radiation Research

- Chemical Physics

- Analytical Chemistry

\section{The National Engineering Laboratory}

Provides technology and technical services to the public and private sectors to address national needs and to solve national problems; conducts research in engineering and applied science in support of these efforts; builds and maintains competence in the necessary disciplines required to carry out this research and technical service; develops engineering data and measurement capabilities; provides engineering measurement traceability services; develops test methods and proposes engineering standards and code changes; develops and proposes new engineering practices; and develops and improves mechanisms to transfer results of its research to the ultimate user. The Laboratory consists of the following centers:

\section{The Institute for Computer Sciences and Technology}

Conducts research and provides scientific and technical services to aid Federal agencies in the selection, acquisition, application, and use of computer technology to improve effectiveness and economy in Government operations in accordance with Public Law 89-306 (40 U.S.C. 759), relevant Executive Orders, and other directives; carries out this mission by managing the Federal Information Processing Standards Program, developing Federal ADP standards guidelines, and managing Federal participation in ADP voluntary standardization activities; provides scientific and technological advisory services and assistance to Federal agencies; and provides the technical foundation for computer-related policies of the Federal Government. The Institute consists of the following centers:
- Applied Mathematics

- Electronics and Electrical Engineering ${ }^{2}$

- Manufacturing Engineering

- Building Technology

- Fire Research

- Chemical Engineering ${ }^{2}$

\section{The Center for Materials Science}

Conducts research and provides measurements, data, standards, reference materials, quantitative understanding and other technical information fundamental to the processing, structure, properties and performance of materials; addresses the scientific basis for new advanced materials technologies; plans research around cross-country scientific themes such as nondestructive evaluation and phase diagram development; oversees Bureau-wide technical programs in nuclear reactor radiation research and nondestructive evaluation; and broadly disseminates generic technical information resulting from its programs. The Center consists of the following Divisions:
- Programming Science and Technology

- Computer Systems Engineering 
$40,4 \sqrt{5}$

\section{Directory of Accredited Laboratories}

Harvey W. Berger, Editor

Office of Product Standards Policy

National Bureau of Standards

Gaithersburg, MD 20899

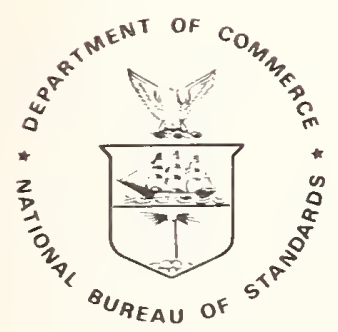

U.S. DEPARTMENT OF COMMERCE, Malcolm Baldrige, Secretary NATIONAL BUREAU OF STANDARDS, Ernest Ambler, Director 
Library of Congress Catalog Card Number: 84-601165

National Bureau of Standards Special Publication 687

Natl. Bur. Stand. (U.S.), Spec. Publ. 687, 79 pages (February 1985) CODEN: XNBSAV 


\section{PREFACE}

The National Bureau of Standards' National Voluntary Laboratory Accreditation Program (NVLAP) improves the competence of testing laboratories and the reliability of laboratory measurements through transfer of measurement technology. Critical elements of test methods are identified along with precision and accuracies expected from the methods when measurements are made. Proficiency testing and interlaboratory comparisons contribute to improved test methods and laboratory performance.

This directory provides information on the activities of the National Bureau of Standards in administering NVLAP during calendar year 1984. Voluntary participation by the Nation's laboratories is increasing and several new accreditation efforts requested by government agencies and private organizations have been established.

The accredited laboratories have been found competent to perform the specific test methods shown in the Directory of Accredited Laboratories. They have the skilled people, necessary facilities and equipment, and documentation and quality assurance systems to produce reliable test data. We recommend that consideration be given to the use of these laboratories whenever their accredited testing capabilities satisfy testing needs.

NVLAP has also provided the basis for acceptance by other countries of test data produced by laboratories in the United States through bilateral agreements. We shall continue to work toward liberalizing the means to satisfying trade requirements whenever possible.

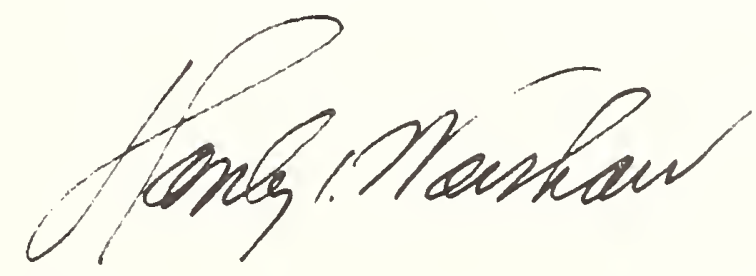

Director

Office of Product Standards Policy 


\section{NVLAP DIRECTORY OF ACCREDITED LABORATORIES}

\section{Contents}

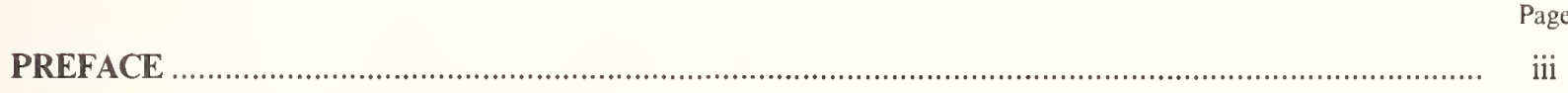

\section{REPORT OF PROGRAM ACTIVITIES}

Introduction

Revision of Procedures

Established Laboratory Accreditation Programs

Insulation

Concrete

Carpet

Stove.

Acoustics

Dosimetry

Commercial Products

Film

Laboratory Participation Summary

APPENDIX Administrative Procedures

NVLAP Accreditation Process

Establishment of New LAPs

\section{INDEXES}

1. Laboratory Name and NVLAP Lab Code Number

2. LAP Name and Laboratories Accredited Under Each LAP

3. Accredited Laboratories by State and NVLAP Code Number

4. Test Methods Available Under Each LAP and NVLAP Code Numbers of Laboratories Accredited for those Test Methods 



\section{REPORT OF PROGRAM ACTIVITIES}

\section{Introduction}

The National Voluntary Laboratory Accreditation Program (NVLAP), administered by the National Bureau of Standards (NBS), was established in 1976 to accredit laboratories for specific tests or types of tests in certain product or service areas where a need for accreditation is determined. As of December 31, 1984, NVLAP has accredited 130 laboratories by laboratory accreditation program (LAP).

Accreditation criteria, which are published as part of the NVLAP procedures, are used for evaluating applicant laboratories. NBS uses periodic on-site assessments, proficiency testing programs, and questionnaires as evaluation tools.

This Directory is the eighth in a series of documents which describe NVLAP program activities and present the list of accredited laboratories and the test methods for which they are accredited.

The beginning of Fiscal Year 1985, on October 1, marked the successful completion of the first phase of NVLAP operations. Since its inception, NVLAP has been provided Federal resources for the development of new LAPs. In keeping with program objectives NVLAP began fully cost reimbursable operations on October 1. Requestors of new LAPs are asked to provide resources for the development of the technical content of new LAPs. In most cases contributing technical assistance, in the development of requirements and criteria, through personal participation rather than funding is needed to initiate a new LAP.

The following sections describe the status of current LAPs, the growth in laboratory participation, and the processes for becoming accredited and requesting new LAPs.

\section{Revision of Procedures}

The NVLAP Procedures underwent the first major revision since the procedures were established in February 1976. NBS published the new NVLAP procedures in the Federal Register on November 8, 1984. The goals of NVLAP remain unchanged, but the requirements for requesting, developing, and establishing laboratory accreditation programs (LAPs) have been significantly changed. Use of the Federal Register has also been minimized.
The goals of NVLAP are to:

(1) Provide national recognition for competent laboratories;

(2) Provide laboratory management with a quality assurance check;

(3) Identify competent laboratories for laboratory users; and

(4) Provide laboratories with guidance from technical experts to improve their performance.

Previous requirements for monthly, quarterly and annual reports, many of which needed to be published in the Federal Register, have been eliminated. Now, a directory of accredited laboratories will be published annually, with periodic supplements to the directory published as necessary.

LAPs will continue to be developed in response to requests and demonstrated need. However, preliminary and final findings of need will no longer be published in the Federal Register. A requestor must still identify the product or service of a proposed LAP and the standards and test methods proposed for inclusion as well as state why the public would benefit, why there is a national need for such a LAP, and what the expected market would be. Interested parties still have the opportunity to comment on the need for any proposed LAP.

In addition to the actions of granting, renewing, denying, and revoking accreditation, NBS will now have the flexibility of suspending a laboratory's accreditation.

Conditions and criteria for accreditation have been clarified and enhanced. The criteria essentially follow the requirements of ISO Guide 25: General Requirements for the Technical Competence of Testing Laboratories. The elements of the criteria address a laboratory's:

(1) quality system;

(2) staff;

(3) facilities and equipment;

(4) calibration;

(5) test methods and procedures;

(6) records; and

(7) test reports.

Overall, the revision is a significant improvement that enables NBS to administer NVLAP more efficiently than was possible under the previous procedures.

\section{Established Laboratory Accreditation Programs}

Laboratories continue to apply for initial accreditation and reaccreditation in the Thermal Insulation, Concrete, Carpet, Stove, and Acoustics LAPs. In addition, 
new LAPs have been established for: laboratories that test paint, paper, or mattresses (the Commercial Products LAP), processors of personnel dosimeters (the Dosimetry LAP), and laboratories that test photographic film (the Film LAP). Laboratories have requested and been accredited under the Commercial and Dosimetry LAPs.

The current participation and accreditable test methods for all established LAPs are given in following sections of this Directory.

\section{Insulation LAP}

The LAP for thermal insulation materials testing has 62 test methods for which a laboratory can seek accreditation. As of December 31, 1984, 36 laboratories were accredited to perform those test methods. Twenty-seven on-site visits were made during the year to accredited laboratories or those seeking accreditation. NBSIR 84-2890 reporting the results of Proficiency Test Round 9 was issued in May 1984. The results of Round 10 will be issued in 1985 .

\section{Concrete LAP}

The LAP for freshly mixed concrete testing has seven test methods covering field testing and laboratory testing. As of December 31, 1984, 31 laboratories were accredited to perform selected test methods. Thirteen on-site visits were made during the year to accredited laboratories or those seeking accreditation. Results of the Concrete LAP between-laboratory proficiency testing program were reported to participants in August 1984.

\section{Carpet LAP}

The LAP for carpet testing has 12 test methods for which a laboratory can seek accreditation. As of December 31, 1984, 24 laboratories were accredited to perform selected test methods. The Department of Housing and Urban Development uses test results produced by these laboratories as part of its carpet certification program. Seventeen on-site visits were made during the year to accredited laboratories or those seeking accreditation. The sixth and seventh rounds of proficiency testing were completed for carpet test methods involving colorfastness, pile weight, pile thickness, strength, and flammability properties. A Tech Brief reporting the results of Round 7 and summarizing the results of Rounds 1 through 7, was issued in February 1984. Round 8 will be issued early in 1985 .

\section{Stove LAP}

The LAP for solid fuel room heaters has 36 test methods, arranged in three groups: (1) a physical/fire test group, (2) a mobile home test group, and (3) an electrical test group, for which a laboratory can seek accreditation. Canadian Standards Association (CSA) Standards B 366.2-M1984, C 22.2 No. 103-1979, and C 22.2 No. 113-1982, have been added to the available test methods. A laboratory may be accredited in any one of 12 options which are various combinations of the three groups and Underwriters Laboratory and CSA standards. As of December 31, 1984, 10 laboratories were accredited to perform selected test methods. Four on-site visits were made during the year to accredited laboratories or those seeking accreditation. A Tech Brief reporting the results of Round 2 Proficiency Testing was issued in November 1984.

\section{Acoustics LAP}

The LAP for acoustical testing services has 49 test methods for which a laboratory can seek accreditation. As of December 31, 1984, eight laboratories were accredited to perform selected test methods. Three on-site visits were made during the year to laboratories seeking accreditation. Data have been collected from participating laboratories for Round 1 of proficiency testing for ASTM test method E 90. A Tech Brief reporting the results of the first round of proficiency testing for C 423-81 was issued in September 1984.

\section{Dosimetry LAP}

The LAP for Personnel Radiation Dosimetry Processors began officially on January 1, 1984. Processors may be accredited in any or all of eight categories. During the year 29 processors participated in proficiency testing in accordance with ANSI N13.11-1983. Successful completion of proficiency testing in each category requested is mandatory to gain accreditation. Twentythree on-site visits were made to processors seeking initial accreditation in this LAP. As of December 31, 1984, 19 processors have been accredited.

\section{Commercial Products LAP}

The LAP for commercial products has a total of 188 test methods: 127 for paint and related materials, 55 for paper and related products, and 6 for mattresses. As of December 31, 1984, 2 laboratories received on-site visits and received initial accreditation to perform selected test methods under the paint section of the LAP. The two laboratories are participating in a proficiency testing program operated by Collaborative Testing Services, Inc. as a requirement for accreditation under the LAP.

\section{Film LAP}

The LAP for photographic film was officially established on August 31, 1984. Several individuals are being considered for selection as technical experts to carry out on-site assessments and laboratory evaluations prior to accreditation.

\section{Laboratory Participation Summary}

The number of laboratories in the system, as of December 31,1984 , categorized by LAP participation is shown below. 
Laboratories in One LAP

Insulation (TIM)

Concrete (CON) .

Carpet (CAR)

Stove (STO)

Acoustics (ACO)

Dosimetry (DOS)

Commercial (CPL)

Film (FLM)

Laboratories in Two LAPs

Insulation and Carpet

Insulation and Acoustics

Insulation and Stove .

Insulation and Concrete

Laboratories in Three LAPs

Insulation, Carpet and Stove

Laboratories in More Than Three LAPs

Total
Number

5
3
1
1

5

3

1

1

1

0

118
The following table summarizes accreditation actions that have occurred during calendar year 1984. Since some laboratories are accredited in more than one LAP, the number of accredited laboratories listed by LAP is greater than the number of laboratories in the system.

\section{LAP Name}

TIM CON CAR STO ACO DOS CPL FLM TOTAL

Voluntary

$\begin{array}{llllllllll}\text { Terminations ... } & 2 & 10 & 1 & 0 & 0 & 0 & 0 & 0 & 13\end{array}$

New Laboratory

$\begin{array}{llllllllll}\text { Accreditations .. } & 6 & 3 & 3 & 0 & 1 & 19 & 2 & 0 & 34\end{array}$

Total Accredited

$\begin{array}{llllllllll}\text { Labs by LAP... } & 36 & 31 & 24 & 10 & 8 & 19 & 2 & 0 & 130\end{array}$

Change in Total

Accredited

Labs from

December,

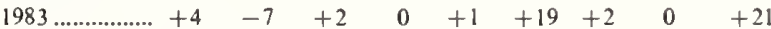




\section{APPENDIX Administrative Procedures}

\section{NVLAP Accreditation Process}

Accreditation is granted following successful completion of a process which includes submission of an application and payment of fees by the laboratory, on-site assessments, proficiency testing, resolution of any identified deficiencies, evaluation, and adisinistrative review.

\section{Criteria and Conditions of Accreditation}

The criteria for accreditation address a laboratory's quality system, staff, facilities and equipment, calibration, test methods and procedures, records, and test reports. Under the conditions of accreditation, a laboratory must limit its test work to those areas where competence and capacity are available and must render test reports objectively and without bias. Evidence found to the contrary is grounds for adverse accreditation action (denial, suspension or revocation).

\section{On-site Assessment}

Before initial accreditation and about every 2 years thereafter, an on-site assessment of each laboratory is conducted to determine compliance with the criteria. Assessors use checklists so that each laboratory receives a fair assessment in relation to others. However, assessors have considerable latitude to make judgments about each laboratory's compliance with the criteria depending on their experience and the unique circumstances of each laboratory. The assessors are selected and assigned on the basis of their expertise in the testing techniques to be reviewed. The time needed to conduct an assessment varies, but 2 days is the norm. Every effort is made to conduct an assessment with as little disruption as possible to the normal operations of the laboratory. The assessors:

(1) Meet with management and supervisory personnel responsible for the laboratory's activities for which accreditation is being sought to acquaint the individuals involved and to set the assessment agenda.

(2) Examine the quality system employed by the laboratory. The history of one or more samples from receipt to final issuance of test reports is traced. Assessors thoroughly review the laboratory's quality manual or equivalent, examine technician notebooks for records pertaining to the samples, check sample identification and tracking procedures, determine whether the appropriate testing conditions are maintained, and examine copies of completed test reports.

(3) Review records of periodic internal audits, use of check samples or participation in round robin testing or other similar programs.
(4) Review representative records including competency evaluations for all staff members who perform the tests, calibration/verification records, and sample control records.

(5) Observe demonstrations of testing techniques and discuss them with the technical personnel to assure their understanding of the procedures.

(6) Examine major equipment, apparatus, and facilities.

At the conclusion of the assessment, an exit briefing is held to discuss assessment findings with laboratory management and identify any deficiencies uncovered. A written summary of all identified deficiencies is left at the laboratory. Assessment forms and a written report are submitted to NBS for further evaluation. The laboratory is asked to respond within 30 days of the date of the exit briefing and provide documentation or certification that the specific deficiencies have been corrected or that specific actions are being taken. Any laboratory applying for initial accreditation may request a delay in responding.

If any deficiencies are noted at laboratories which are currently accredited, such deficiencies must be corrected within 30 days after the exit briefing or the laboratory may face possible suspension, revocation or expiration of its accreditation. When test equipment is identified as out-of-calibration, it must not be used until corrective action has been completed. Any deficiencies noted for corrective action will be subject to thorough review and verification during subsequent assessments.

\section{Monitoring Visits}

In addition to regularly scheduled assessments, monitoring visits can be made at any time during the accreditation period. Monitoring visits may occur for cause or on a random selection basis. These visits serve to verify reported changes in the laboratory's personnel, facilities, and operations or to explore possible reasons for poor performance in proficiency testing. The scope of a monitoring visit may range from checking a few designated items to a complete review. Failure to cooperate with NVLAP assessors may be grounds for adverse accreditation action. No additional fee is required for the monitoring visit since the cost is already factored into the fees.

\section{Proficiency Testing}

Proficiency testing is an integral part of the NVLAP accreditation process. While the existence of facilities, equipment, and personnel which satisfy the criteria indicates a laboratory's overall capability to obtain good results, an analysis of actual test results for certain test 
methods is also necessary to determine if the overall capability does in fact produce the desired results. A laboratory's failure to participate fully in the conduct of required proficiency testing is grounds for adverse accreditation action.

\section{Evaluation}

Evaluation of a laboratory is conducted at NBS by technical experts chosen for their experience and knowledge of the test methods under evaluation. They review records on each applicant laboratory and base their evaluation on:

(1) Information provided on the application;

(2) On-site assessment reports;

(3) Actions taken by the laboratory to correct deficiencies;

(4) Results of proficiency testing; and

(5) Information from any monitoring visits of the laboratory.

If the technical evaluation reveals additional deficiencies, written notification describing them will be made to the laboratory. The laboratory must respond within 30 days of such notification and provide documentation or certification that the specified deficiencies have been corrected. Clarification of some issues may be requested by telephone. All deficiencies must be corrected before accreditation can be granted or renewed.

\section{Technical Experts}

The technical experts (respected peers in their field) used as assessors and evaluators are selected through evaluation of their professional/academic achievements, experience in the field of testing, management awareness, potential for conflict-of-interest, and tact in dealing with people.

Assessors are selected to conduct an on-site assessment of a particular laboratory on the basis of how well their individual experience matches the type of testing to be assessed. The laboratory has the right to appeal the assignment of an assessor and may request an alternate.

Evaluators are selected to provide a second opinion, if necessary, and to review the records including the application, assessment report, deficiencies, corrections to deficiencies, and proficiency test results and, based on this record, to recommend whether accreditation should be granted.

\section{Administrative Review}

When the evaluation has been completed, NBS prepares an administrative recommendation that the laboratory either be granted or denied accreditation. This recommendation is based on a review of the evaluation and other records to ensure that all NVLAP technical, financial, and administrative obligations have been satisfied.

\section{Accreditation Actions}

The Director of the NBS Office of Product Standards Policy makes accreditation decisions.

Recommended. When accreditation is recommended, the recommendation forms the basis for granting accreditation. A certificate of accreditation is issued to the laboratory.

Denial. In cases where denial is recommended, the laboratory is notified of a proposal to deny accreditation and the reasons for the denial.

Appeal. When denial has been proposed, the laboratory may request a hearing, under 5 United States Code (U.S.C.) 556, within 30 days of the date of receipt of the notification. If a hearing is not requested, the denial becomes final upon the expiration of that 30-day period.

Renewal. Accreditation is granted annually or biennially with renewal occurring on the same anniversary date every year or every two years.

Termination. A laboratory may voluntarily terminate its accreditation by written request at any time. The accreditation certificate must be returned with the request. If a laboratory elects not to renew its accreditation, a notification of such intention should be forwarded to NBS in writing.

Suspension. If an accredited laboratory develops problems or deficiencies which are of a temporary nature, its accreditation may be suspended until such time as the deficiencies are resolved.

Revocation. In cases where a laboratory is found to have violated the terms of its accreditation, the accreditation can be revoked. The laboratory may, however, be given the option to voluntarily terminate accreditation. The laboratory has 30 days from the date of receipt of notice of proposed revocation in which it may appeal the proposed revocation by requesting a hearing. If a hearing is not requested, the revocation becomes final upon the expiration of that 30-day period. When revocation is final the laboratory must return its certificate of accreditation and cease to reference its NVLAP accreditation on any of its reports, other correspondence, or advertising.

\section{Public Notification}

Accreditation actions are published quarterly. A directory of accredited laboratories is published annually. The directofy identifies the name and address of each laboratory, the scope of its accreditation, and the key contact person. The directory is widely distributed nationally and internationally.

Accredited laboratories are encouraged to publicize their accredited status. However, they must do so in such a way as not to imply product certification by NBS. A laboratory may cite its accredited status and use the NVLAP logo on reports, stationary, and in business and trade publications. 


\section{Establishment of New LAPs}

Anyone may request a LAP by writing to the Director, NBS, ADMIN A1134, Gaithersburg, MD 20899. The letter must address the following items:

(1) The scope of the LAP in terms of the products or testing services proposed for inclusion.

(2) Specific identification of the applicable standards and test methods including appropriate designations, and the organizations or standards writing bodies having responsibility for them;

(3) A statement of need for the LAP including:

(i) Technical and economic reasons why the LAP would benefit the public' interest.

(ii) Evidence of a national need to accredit testing laboratories for the specific scope beyond that served by an existing laboratory accreditation program in the public or private sector.

(iii) An estimate of the number of laboratories that may seek accreditation.

(iv) An estimate of the number and nature of the users of such laboratories.

(4) A statement of the extent to which you are willing to support necessary developmental aspects of the LAP with funding and personnel.

If the request letter addresses the above items, NBS will publish a Federal Register notice of the receipt of a LAP request describing the scope of the requested LAP, and stating that anyone may submit comments on the need for the LAP to NBS. Assuming there is public support for such a LAP, the process of securing resources for its successful development can begin. 
This directory is current as of December 31, 1984

\section{ACCREDITED LABORATORIES AND TEST METHODS FOR WHICH THEY ARE ACCREDITED}

NOTE: This section lists accredited laboratories in ascending order by NVLAP Lab Code Number. Indexes 1, 2,3 , and 4 are lists of laboratories by test method, state, and laboratory accreditation program (LAP) crossreferenced to NVLAP Lab Code Number.

NVLAP LAB CODE 0101

\section{CERTAINTEED CORPORATION INSULATION GROUP, R \& D LABORATORY \\ 1400 Union Meeting Road, Blue Bell, PA 19422 \\ Dr. W. Francis Olix Phone: 215-341-6713}

Accreditation Renewal Date: January 1, 1986

$\begin{array}{ll}\text { NVLAP Code } & \begin{array}{l}\text { Designation } \\ \text { HH-I-515 } \\ \text { (para. 4.8.5 in D version, }\end{array} \\ \text { Amendment 1) } \\ 01 / \text { C03 } & \\ & \\ 01 / \text { D01 } & \text { ASTM C136 } \\ 01 / \text { D02 } & \text { ASTM C167 } \\ 01 / \text { D08 } & \text { ASTM C302 } \\ 01 / \text { D09 } & \text { ASTM C303 } \\ 01 / \text { D13 } & \text { ASTM C519 } \\ 01 / \text { D25 } & \text { HH-I-515 } \\ & \text { (para. 4.8.3 in D version, } \\ & \text { Amendment 1) } \\ 01 / \text { D26 } & \text { HH-I-515 } \\ & \text { (para. 4.8.1 in D version, } \\ & \text { Amendment 1) } \\ 01 / \text { F01 } & \text { TAPPI T461 } \\ 01 / \text { F05 } & \text { ASTM E136 } \\ 01 / \text { F07 } & \text { HH-I-515 } \\ & \text { (para. 4.8.7 in D version, } \\ & \text { Amendment 1) } \\ 01 / \text { F08 } & \text { HH-I-515 } \\ & \text { (para. 4.8.8 in D version, } \\ & \text { Amendment 1) } \\ 01 / \text { S01 } & \text { ASTM C165 } \\ 01 / \text { S08 } & \text { ASTM C446 } \\ 01 / \text { S09 } & \text { ASTM D781 } \\ 01 / \text { S10 } & \text { ASTM D828 } \\ 01 / \text { S12 } & \\ 01 / \text { T01 } & \text { ASTM C177 } \\ & \end{array}$

Short Title

Corrosiveness; Cellulosic fiber (loose-fill)

California Energy Commission tests for insulating materials:

Corrosiveness - Mineral fiber blankets and loose-fill

Sieve or screen analysis

Thickness and density; Blanket and batt

Density; Preformed pipe insulation

Density; Preformed block insulation

Density; Loose-fill (fibrous)

Moisture absorption;

Cellulosic fiber (loose-fill)

Settled density; Cellulosic fiber (loose-fill)

Flame Resistance; Paper and paperboard

Behavior of Materials in a Vertical Tube Furnace

Critical radiant flux;

Radiant Panel (cellulosic fiber, loose-fill)

Smoldering combustion;

Cellulosic fiber (loose-fill)

Compressive properties; Thermal insulation (proc. A)

Breaking load/modulus of rupture; Preformed pipe insulation

Puncture test; Paperboard and fiberboard

Tensile breaking strength; Paper and paperboard

California Energy Commission tests for insulating materials:

Bond strength - Spray applied cellulose

Thermal transmission properties; Low-temperature guarded hot plate 


$\begin{array}{lll}\text { NVIAP Code } & \text { Designation } & \text { Short Title } \\ \text { 01/T04 } & \text { ASTM C236 } & \text { Thermal conductance; Guarded hot box } \\ \text { 01/T05 } & \text { ASTM C335 } & \text { Thermal conductivity; Pipe insulation } \\ 01 / \text { T06 } & \text { ASTM C518 } & \text { Thermal transmission properties; Heat flow meter } \\ 01 / \text { T09 } & \text { ASTM C653 } & \text { Thermal resistance (Rec. Practice); Blanket (mineral fiber) } \\ 01 / \text { T10 } & \text { ASTM C687 } & \text { Thermal resistance (Rec. Practice); Loose-fill (fibrous) } \\ 01 / \text { V04 } & \text { ASTM E96 } & \text { Water vapor transmission; Thin sheets (proc. A) }\end{array}$

NVLAP LAB CODE 0102

\section{BUTLER MANUFACTURING COMPANY RESEARCH CENTER}

135th Street and Botts Road, Grandview, MO 64030

Marvin K. Snyder Phone: 816-763-3022

Accreditation Renewal Date: January 1, 1986

$\begin{array}{ll}\text { NVLAP Code } & \text { Designation } \\ 01 / \text { T04 } & \text { ASTM C236 } \\ 01 / \text { T06 } & \text { ASTM C518 }\end{array}$

Short Title

Thermal conductance; Guarded hot box

Thermal transmission properties; Heat flow meter

NVLAP LAB CODE 0103

\section{DOW CHEMICAL USA, FOAM PRODUCTS RESEARCH PRODUCT EVALUATION GROUP \\ P.O. Box 515, Granville, $\mathrm{OH} 43023$ \\ M.J. Ennis Phone: 614-587-4313}

Accreditation Renewal Date: January 1, 1986

$\begin{array}{ll}\text { NVLAP Code } & \text { Designation } \\ 01 / \text { D18 } & \text { ASTM D1622 } \\ 01 / \text { D21 } & \text { ASTM D2126 } \\ 01 / \text { D23 } & \text { ASTM D2842 } \\ 01 / \text { D27 } & \text { ASTM D2126 } \\ 01 / \text { S02 } & \text { ASTM C203 } \\ 01 / \text { S07 } & \text { ASTM C273 } \\ 01 / \text { S11 } & \text { ASTM D1621 } \\ 01 / \text { T06 } & \text { ASTM C518 } \\ 01 / \text { V04 } & \text { ASTM E96 }\end{array}$

Short Title

Apparent density; Rigid cellular plastics

Response to thermal and humid aging (proc. E); Rigid cellular plastics

Water absorption; Rigid cellular plastics

Response to thermal and humid aging (proc. C); Rigid cellular plastics

Breaking load/flexural strength; Preformed block insulation

Shear test; Sandwich construction

Compressive properties; Rigid cellular plastics (proc. A-Crosshead)

Thermal transmission properties; Heat flow meter

Water vapor transmission; Thin sheets (proc. A)

NVLAP LAB CODE 0104

NAHB RESEARCH FOUNDATION, INC.

P.O. Box 1627, Rockville, MD 20850

Hugh Angleton Phone: 301-762-4200

Accreditation Renewal Date: January 1, 1986

$\begin{array}{ll}\text { NVLAP Code } & \text { Designation } \\ 01 / \text { D02 } & \text { ASTM C167 } \\ 01 / \text { D13 } & \text { ASTM C519 } \\ 01 / \text { T06 } & \text { ASTM C518 } \\ 01 / \text { T09 } & \text { ASTM C653 } \\ 01 / \text { T10 } & \text { ASTM C687 }\end{array}$

Short Title

Thickness and density; Blanket and batt

Density; Loose-fill (fibrous)

Thermal transmission properties; Heat flow meter

Thermal resistance (Rec. Practice); Blanket (mineral fiber)

Thermal resistance (Rec. Practice); Loose-fill (fibrous) 
UNITED STATES TESTING COMPANY, INC.

1415 Park Avenue, Hoboken, NJ 07030

Carl B. Yoder

Phone: 201-792-2400

Accreditation Renewal Date: January 1, 1986

$\begin{array}{ll}\text { NVLAP Code } & \begin{array}{l}\text { Designation } \\ \text { 01/F02 }\end{array} \\ \text { ASTM E84 } & \text { HH-I-515 } \\ & \text { (para. 4.8.7 in D version, } \\ & \text { Amendment 1) } \\ \text { 01/T06 } & \text { ASTM C518 } \\ 03 / \text { C01 } & \text { AATCC 16E } \\ 03 / \text { C02 } & \text { AATCC 8 } \\ \text { 03/D01 } & \text { ASTM D418 } \\ & \\ & \\ & \\ 03 / \text { D02 } & \text { DDD-C-95A } \\ 03 / \text { S01 } & \text { ASTM D1335 } \\ & \text { Federal Test Method } \\ & \text { Standard 191-5100 } \\ & \text { 191-5950 } \\ \text { 03/F01 } & \text { ASTM E84 } \\ \text { 03/F03 } & \text { DoC FF 1-70 } \\ 03 / F 04 & \text { ASTM E648 } \\ 03 / \text { B02 } & \text { UM 44C }\end{array}$

Short Title

Surface burning characteristics; Building materials

Critical radiant flux;

Radiant Panel (cellulosic fiber, loose-fill)

Thermal transmission properties; Heat flow meter

Colorfastness to Light (Xenon Arc)

Colorfastness to Crocking

Pile Yarn Floor Covering Construction

Pile Weight - Uncoated (Section 8)

Pile Weight - Coated (Section 9)

Pile Thickness - (Sections 10 \& 11)

Tuft Height - (Section 13)

Shrinkage

Tuft Bind of Floor Coverings

Textile Test Method - Breaking Strength

Textile Test Method - Delamination

Surface Flammability (Carpet)

Methenamine Pill Test

Radiant Panel (Carpet)

Addenda 2 and 3 Attached Cushion Tests

NVLAP LAB CODE 0106

\section{UNITED STATES TESTING COMPANY, INC. CALIFORNIA DIVISION}

5555 Telegraph Road, Los Angeles, CA 90040

Bernd Givon Phone: 213-723-7181

Accreditation Renewal Date: January 1, 1986

$\begin{array}{ll}\text { NVLAP Code } & \begin{array}{l}\text { Designation } \\ \text { 01/C02 }\end{array} \\ & \begin{array}{l}\text { HH-I-515 } \\ \text { (para. 4.8.5 in D version, }\end{array} \\ \text { Amendment 1) } \\ 01 / \text { D21 } & \text { ASTM D2126 } \\ 01 / \text { D27 } & \text { ASTM D2126 } \\ 01 / \text { D28 } & \text { ASTM D2126 } \\ 01 / \text { F02 } & \text { ASTM E84 } \\ 01 / \text { F05 } & \text { ASTM E136 } \\ 01 / \text { F07 } & \text { HH-I-515 } \\ & \text { (para. 4.8.7 in D version, } \\ & \text { Amendment 1) } \\ 01 / \text { V04 } & \text { ASTM E96 } \\ 03 / \text { C01 } & \text { AATCC 16E } \\ 03 / \text { D01 } & \text { ASTM D418 }\end{array}$

Short Title
Corrosiveness; Cellulosic fiber (loose-fill)

Response to thermal and humid aging (proc. E); Rigid cellular plastics

Response to thermal and humid aging (proc. C); Rigid cellular plastics

Response to thermal and humid aging (proc. G); Rigid cellular plastics

Surface burning characteristics; Building materials

Behavior of Materials in a Vertical Tube Furnace

Critical radiant flux; Radiant Panel (cellulosic fiber, loose-fill)

Water vapor transmission; Thin sheets (proc. A)

Colorfastness to Light (Xenon Arc)

Pile Yarn Floor Covering Construction

Pile Weight - Uncoated (Section 8)

Pile Weight - Coated (Section 9)

Pile Thickness - (Sections 10 \& 11)

Tuft Height - (Section 13)

03/F01 ASTM E84

Surface Flammability (Carpet) 


$\begin{array}{lll}\text { NVLAP Code } & \text { Designation } & \text { Short Title } \\ \text { 03/F03 } & \text { DoC FF 1-70 } & \text { Methenamine Pill Test } \\ \text { 03/F04 } & \text { ASTM E648 } & \text { Radiant Panel (Carpet) }\end{array}$

NVLAP LAB CODE 0107

\section{UNITED STATES TESTING COMPANY, INC. TULSA DIVISION \\ 1341 North 108th East Avenue, Tulsa, OK 74116 \\ Freã D. Wampnar Phone: $918-437-8333$}

Accreditation Renewal Date: January 1, 1986

\begin{tabular}{|c|c|c|}
\hline NVLAP Code & Designation & Short Title \\
\hline $01 / \mathrm{C} 02$ & $\begin{array}{l}\mathrm{HH}-\mathrm{I}-515 \\
\text { (para. } 4.8 .5 \text { in D version, } \\
\text { Amendment 1) }\end{array}$ & Corrosiveness; Cellulosic fiber (loose-fill) \\
\hline $01 / \mathrm{D} 18$ & ASTM D1622 & Apparent density; Rigid cellular plastics \\
\hline $01 / \mathrm{D} 25$ & $\begin{array}{l}\mathrm{HH}-\mathrm{I}-515 \\
\text { (para. } 4.8 .3 \text { in D version, } \\
\text { Amendment } 1 \text { ) }\end{array}$ & $\begin{array}{l}\text { Moisture absorption; } \\
\text { Cellulosic fiber (loose-fill) }\end{array}$ \\
\hline $01 / \mathrm{D} 26$ & $\begin{array}{l}\mathrm{HH}-\mathrm{I}-515 \\
\text { (para. } 4.8 .1 \text { in D version, } \\
\text { Amendment 1) }\end{array}$ & Settled density; Cellulosic fiber (loose-fill) \\
\hline $01 /$ F08 & $\begin{array}{l}\text { HH-I-515 } \\
\text { (para. } 4.8 .8 \text { in D version, } \\
\text { Amendment } 1 \text { ) }\end{array}$ & Smoldering combustion; Cellulosic fiber (loose-fill) \\
\hline 01/V04 & ASTM E96 & Water vapor transmission; Thin sheets (proc. A) \\
\hline 01/V05 & $\begin{array}{l}\text { HH-I-515 } \\
\text { (para. } 4.8 .6 \text { in D version, } \\
\text { Amendment } 1 \text { ) }\end{array}$ & Fungus; Cellulosic fiber (loose-fill) \\
\hline 01/V06 & $\begin{array}{l}\mathrm{HH}-\mathrm{I}-515 \\
\text { (para. } 4.8 .9 \text { in D version, } \\
\text { Amendment } 1 \text { ) }\end{array}$ & Starch; Cellulosic fiber (loose-fill) \\
\hline
\end{tabular}

NVLAP LAB CODE 0108

CERTIFIED TESTING LABORATORIES, INC.

1105 Riverbend Drive, P.O. Box 2041, Dalton, GA 30720

John H. Frank Phone: 404-226-1400

Accreditation Renewal Date: January 1, 1986

\begin{tabular}{|c|c|c|}
\hline NVLAP Code & Designation & Short Title \\
\hline 03/C01 & AATCC $16 \mathrm{E}$ & Colorfastness to Light (Xenon Arc) \\
\hline 03/C02 & AATCC 8 & Colorfastness to Crocking \\
\hline 03/D01 & ASTM D418 & $\begin{array}{l}\text { Pile Yarn Floor Covering Construction } \\
\text { Pile Weight - Uncoated (Section 8) } \\
\text { Pile Weight - Coated (Section 9) } \\
\text { Pile Thickness - (Sections 10 \& 11) } \\
\text { Tuft Height - (Section 13) }\end{array}$ \\
\hline 03/D02 & DDD-C-95A & Shrinkage \\
\hline 03/S01 & $\begin{array}{l}\text { ASTM D1335 } \\
\text { Federal Test Method }\end{array}$ & Tuft Bind of Floor Coverings \\
\hline & $\begin{array}{l}\text { Standard 191-5100 } \\
191-5950\end{array}$ & $\begin{array}{l}\text { Textile Test Method - Breaking Strength } \\
\text { Textile Test Method - Delamination }\end{array}$ \\
\hline 03/E01 & AATCC 134/CRI 102 & Electrostatic Propensity of Carpets \\
\hline 03/F03 & DoC FF 1-70 & Methenamine Pill Test \\
\hline
\end{tabular}




$\begin{array}{ll}\text { NVLAP Code } & \text { Designation } \\ \text { 03/F04 } & \text { ASTM E648 } \\ \text { 03/B02 } & \text { UM 44C }\end{array}$

Short Title

Radiant Panel (Carpet)

Addenda 2 and 3 Attached Cushion Tests

NVLAP LAB CODE 0109

\author{
OWENS-CORNING FIBERGLAS CORPORATION \\ TECHNICAL CENTER LABORATORY \\ P.O. Box 415, Route 16, Granville, $\mathrm{OH} 43023$ \\ William M. Edmunds \\ Ron Moulder \\ Phone: 614-587-7024-For Insulation LAP \\ Phone: 614-587-7066-For Acoustics LAP
}

Accreditation Renewal Date: January 1, 1986'

\begin{tabular}{|c|c|}
\hline NVLAP Code & Designation \\
\hline $01 / \mathrm{C} 01$ & $\begin{array}{l}\text { ASTM C739 } \\
\text { (para. } 10.7 \text { in } 80 \text { version) }\end{array}$ \\
\hline $01 / \mathrm{C} 02$ & $\begin{array}{l}\text { HH-I-515 } \\
\text { (para. } 4.8 .5 \text { in D version, } \\
\text { Amendment } 1 \text { ) }\end{array}$ \\
\hline $01 / \mathrm{C} 03$ & \\
\hline 01/D01 & ASTM C136 \\
\hline 01/D02 & ASTM C167 \\
\hline 01/D03 & $\begin{array}{l}\text { ASTM C209 } \\
\text { (para. } 6 \text { in } 72 \text { version) }\end{array}$ \\
\hline 01/D04 & ASTM C209 \\
\hline 01/D05 & $\begin{array}{l}\text { ASTM C209 } \\
\text { (para. } 13 \text { in } 72 \text { version) } \\
\text { (para. } 100-106 \text { in } 78 \text { version) }\end{array}$ \\
\hline 01/D06 & $\begin{array}{l}\text { ASTM C209 } \\
\text { (para. } 14 \text { in } 72 \text { version) } \\
\text { (para. } 107-110 \text { in } 72 \text { version) }\end{array}$ \\
\hline 01/D07 & ASTM C 272 \\
\hline $01 / \mathrm{D} 08$ & ASTM C302 \\
\hline 01/D09 & ASTM C303 \\
\hline 01/D11 & ASTM C356 \\
\hline $01 / \mathrm{D} 12$ & ASTM C411 \\
\hline $01 / D 13$ & ASTM C519 \\
\hline 01/D15 & ASTM D756 \\
\hline 01/D16 & ASTM D756 \\
\hline $01 / \mathrm{D} 17$ & ASTM D756 \\
\hline $01 / \mathrm{D} 18$ & ASTM D1622 \\
\hline 01/D19 & ASTM D2126 \\
\hline $01 / \mathrm{D} 20$ & ASTM D2126 \\
\hline $01 / \mathrm{D} 21$ & ASTM D2126 \\
\hline $01 / \mathrm{D} 22$ & ASTM D2126 \\
\hline $01 / \mathrm{D} 23$ & ASTM D2842 \\
\hline $01 / \mathrm{D} 24$ & $\begin{array}{l}\text { ASTM C } 739 \\
\text { (para. } 10.5 \text { in } 80 \text { version) }\end{array}$ \\
\hline $01 / \mathrm{D} 25$ & $\begin{array}{l}\text { HH-I-515 } \\
\text { (para. } 4.8 .3 \text { in D version, } \\
\text { Amendment } 1 \text { ) }\end{array}$ \\
\hline 01/D26 & $\begin{array}{l}\text { HH-I-515 } \\
\text { (para. } 4.8 .1 \text { in D version, } \\
\text { Amendment } 1 \text { ) }\end{array}$ \\
\hline $01 / \mathrm{D}$ & ASTM D2126 \\
\hline
\end{tabular}

\title{
Short Title
}

Corrosiveness; Cellulosic fiber (loose-fill)

Corrosiveness; Cellulosic fiber (loose-fill)

California Energy Commission tests for insulating materials:

Corrosiveness - Mineral fiber blankets and loose-fill

Sieve or screen analysis

Thickness and density; Blanket and batt

Thickness; Board (cellulosic fiber)

Water absorption, 2 hour;

Water absorption, 24 hour;

Board (cellulosic fiber) by D1037

Linear expansion; Board (cellulosic fiber) by D1037

Density; Preformed block insulation

Density; Preformed pipe insulation

Density; Preformed block insulation

Linear shrinkage; Soaking heat; Preformed high temperature insulation

Hot-surface performance; High temperature insulation

Density; Loose-fill (fibrous)

Weight and shape changes; Accelerated service (proc. A); Plastics

Weight and shape changes; Accelerated service (proc. B); Plastics

Weight and shape changes; Accelerated service (proc. E); Plastics

Apparent density; Rigid cellular plastics

Response to thermal and humid aging (proc. B); Rigid cellular plastics

Response to thermal and humid aging (proc. D); Rigid cellular plastics

Response to thermal and humid aging (proc. E); Rigid cellular plastics

Response to thermal and humid aging (proc. F); Rigid cellular plastics

Water absorption; Rigid cellular plastics

Moisture absorption; Cellulosic fiber (loose-fill)

Moisture absorption;

Cellulosic fiber (loose-fill)

Settled density; Cellulosic fiber (loose-fill)

Response to thermal and humid aging (proc. C); Rigid cellular plastics 


\begin{tabular}{|c|c|}
\hline $\begin{array}{l}\text { NVLAP Code } \\
\text { 01/D28 } \\
\text { 01/D29 }\end{array}$ & $\begin{array}{l}\text { Designation } \\
\text { ASTM D2126 }\end{array}$ \\
\hline 01/F01 & TAPPI T461 \\
\hline 01/F02 & ASTM E84 \\
\hline $01 /$ F05 & ASTM E136 \\
\hline 01/F07 & $\begin{array}{l}\text { HH-I-515 } \\
\text { (para. 4.8.7 in D version, } \\
\text { Amendment } 1 \text { ) }\end{array}$ \\
\hline 01/F08 & $\begin{array}{l}\mathrm{HH}-\mathrm{I}-515 \\
\text { (para. } 4.8 .8 \text { in D version, } \\
\text { Amendment } 1 \text { ) }\end{array}$ \\
\hline $01 / \mathrm{S} 01$ & ASTM C165 \\
\hline $01 / \mathrm{S} 02$ & ASTM C203 \\
\hline $01 / \mathrm{S} 03$ & $\begin{array}{l}\text { ASTM C209 } \\
\text { (para. } 9 \text { in } 72 \text { version) }\end{array}$ \\
\hline $01 / \mathrm{S} 04$ & $\begin{array}{l}\text { ASTM C } 209 \\
\text { (para. } 10 \text { in } 72 \text { version) }\end{array}$ \\
\hline $01 / \mathrm{S} 05$ & $\begin{array}{l}\text { ASTM C209 } \\
\text { (para. } 11 \text { in } 72 \text { version) }\end{array}$ \\
\hline $01 / \mathrm{S} 06$ & $\begin{array}{l}\text { ASTM C209 } \\
\text { (para. } 12 \text { in } 72 \text { version) }\end{array}$ \\
\hline 01/S07 & ASTM C 273 \\
\hline $01 / \mathrm{S} 08$ & ASTM C446 \\
\hline $01 / \mathrm{S} 09$ & ASTM D781 \\
\hline $01 / \mathrm{S} 10$ & ASTM D828 \\
\hline $01 / \mathrm{S} 11$ & ASTM D1621 \\
\hline $01 / \mathrm{T} 01$ & ASTM C177 \\
\hline $01 /$ T04 & ASTM C236 \\
\hline $01 /$ T05 & ASTM C 335 \\
\hline $01 /$ T06 & ASTM C518 \\
\hline $01 /$ T09 & ASTM C653 \\
\hline $01 / \mathrm{T} 10$ & ASTM C687 \\
\hline $01 / \mathrm{V} 02$ & TAPPI T419 \\
\hline 01/V03 & ASTM D2020 \\
\hline 01/V04 & ASTM E96 \\
\hline $01 / \mathrm{V} 05$ & HH-I-515 \\
\hline
\end{tabular}

(para. 4.8.6 in D version, Amendment 1)

08/P01 ASTM C367-78

08/P02 ASTM C384-77(84)

08/P03 ASTM C423-84a

08/P04 ASTM C522-80

08/P05 ASTM C523-68 (81)

08/P06 ASTM E90-83

08/P10 ANSI S1.31-80

08/P13 ANSI S1.32-80

08/E21 AMA-1-II-67
Short Title

Response to thermal and humid aging (proc. G); Rigid cellular plastics

California Energy Commission tests for insulating materials:

Installed compressed thickness

Flame Resistance; Paper and paperboard

Surface burning characteristics; Building materials

Behavior of Materials in a Vertical Tube Furnace

Critical radiant flux; Radiant Panel (cellulosic fiber, loose-fill)

Smoldering combustion; Cellulosic fiber (loose-fill)

Compressive properties; Thermal insulation (proc. A)

Breaking load/flexural strength; Preformed block insulation

Transverse strength; Board (cellulosic fiber)

Deflection at specified load; Board (cellulosic fiber)

Tensile strength; Parallel to surface; Board (cellulosic fiber)

Tensile strength; Perpendicular to surface

Shear test; Sandwich construction

Breaking load/modulus of rupture; Preformed pipe insulation

Puncture test; Paperboard and fiberboard

Tensile breaking strength; Paper and paperboard

Compressive properties; Rigid cellular plastics (proc. A-Crosshead)

Thermal transmission properties; Low-temperature guarded hot plate

Thermal conductance; Guarded hot box

Thermal conductivity; Pipe insulation

Thermal transmission properties; Heat flow meter

Thermal resistance (Rec. Practice); Blanket (mineral fiber)

Thermal resistance (Rec. Practice); Loose-fill (fibrous)

Starch in paper; Qualitative test

Mildew (fungus) resistance; Paper and paperboard

Water vapor transmission; Thin sheets (proc. A)

Fungus; Cellulosic fiber (loose-fill)

Strength Properties, Prefabricated Architectural Acoustical Materials Impedance and Absorption of Acoustical Materials

Sound Absorption and Sound Absorption Coefficients

Airflow Resistance of Acoustical Materials

Light Reflectance of Acoustical Materials

Airborne Sound Transmission Loss of Building Partitions

Sound Power Levels, Broad-Band Noise Sources in Reverberation

Rooms $(100-10,000 \mathrm{~Hz})$

Sound Power Levels, Discrete- Frequency and Narrow-Band

Noise Sources in Reverberation Rooms $(100-10,000 \mathrm{~Hz})$

Ceiling Sound Transmission Test by Two-Room Method 


\section{JIM WALTER RESEARCH CORPORATION \\ 10301 9th Street North, St. Petersburg, FL 33702 \\ Alan P. Conroy Phone: 813-576-4171}

Accreditation Renewal Date: January 1, 1986

\begin{tabular}{|c|c|}
\hline NVLAP Code & Designation \\
\hline 01/D03 & $\begin{array}{l}\text { ASTM C209 } \\
\text { (para. } 6 \text { in } 72 \text { version) }\end{array}$ \\
\hline 01/D04 & ASTM C209 \\
\hline 01/D05 & $\begin{array}{l}\text { ASTM C } 209 \\
\text { (para. } 13 \text { in } 72 \text { version) } \\
\text { (para. } 100-106 \text { in } 78 \text { version) }\end{array}$ \\
\hline 01/D06 & $\begin{array}{l}\text { ASTM C209 } \\
\text { (para. } 14 \text { in } 72 \text { version) } \\
\text { (para. } 107-110 \text { in } 72 \text { version) }\end{array}$ \\
\hline 01/D07 & ASTM C272 \\
\hline 01/D09 & ASTM C303 \\
\hline 01/D20 & ASTM D2126 \\
\hline 01/D21 & ASTM D2126 \\
\hline 01/F02 & ASTM E84 \\
\hline 01/S02 & ASTM C203 \\
\hline $01 / \mathrm{S} 03$ & $\begin{array}{l}\text { ASTM C209 } \\
\text { (para. } 9 \text { in } 72 \text { version) }\end{array}$ \\
\hline 01/S04 & $\begin{array}{l}\text { ASTM C209 } \\
\text { (para. } 10 \text { in } 72 \text { version) }\end{array}$ \\
\hline $01 / \mathrm{S} 05$ & $\begin{array}{l}\text { ASTM C209 } \\
\text { (para. } 11 \text { in } 72 \text { version) }\end{array}$ \\
\hline $01 / \mathrm{S} 06$ & $\begin{array}{l}\text { ASTM C209 } \\
\text { (para. } 12 \text { in } 72 \text { version) }\end{array}$ \\
\hline 01/S11 & ASTM D1621 \\
\hline 01/T01 & ASTM C177 \\
\hline 01/T04 & ASTM C236 \\
\hline 01/T05 & ASTM C335 \\
\hline 01/T06 & ASTM C518 \\
\hline 01/V04 & ASTM E96 \\
\hline 08/P02 & ASTM C38477(84) \\
\hline 08/P03 & ASTM C423-84a \\
\hline 08/P06 & ASTM E90-83 \\
\hline $08 / \mathrm{E} 21$ & AMA-1-II-67 \\
\hline
\end{tabular}

Short Title

Thickness; Board (cellulosic fiber)

Water absorption, 2 hour;

Water absorption, 24 hour; Board (cellulosic fiber) by D1037

Linear expansion; Board (cellulosic fiber) by D1037

Water absorption; Core materials

Density; Preformed block insulation

Response to thermal and humid aging (proc. D); Rigid cellular plastics Response to thermal and humid aging (proc. E); Rigid cellular plastics

Surface burning characteristics; Building materials

Breaking load/flexural strength; Preformed block insulation

Transverse strength; Board (cellulosic fiber)

Deflection at specified load; Board (cellulosic fiber)

Tensile strength; Parallel to surface; Board (cellulosic fiber)

Tensile strength; Perpendicular to surface

Compressive properties; Rigid cellular plastics (proc. A-Crosshead)

Thermal transmission properties; Low-temperature guarded hot plate

Thermal conductance; Guarded hot box

Thermal conductivity; Pipe insulation

Thermal transmission properties; Heat flow meter

Water vapor transmission; Thin sheets (proc. A)

Impedance and Absorption of Acoustical Materials

Sound Absorption and Sound Absorption Coefficients

Airborne Sound Transmission Loss of Building Partitions

Ceiling Sound Transmission Test by Two-Room Method

NVLAP LAB CODE 0113

\section{DYNATECH R/D COMPANY THERMOPHYSICS LABORATORY \\ 99 Erie Street, Cambridge, MA 02139 \\ Andre O. Desjarlais Phone: 617-868-8050}

Accreditation Renewal Date: January 1, 1986

$\begin{array}{ll}\text { NVLAP Code } & \text { Designation } \\ 01 / \text { T01 } & \text { ASTM C177 } \\ 01 / \text { T04 } & \text { ASTM C236 } \\ 01 / \text { T05 } & \text { ASTM C335 } \\ 01 / \text { T06 } & \text { ASTM C518 }\end{array}$

Short Title

Thermal transmission properties; Low-temperature guarded hot plate

Thermal conductance; Guarded hot box

Thermal conductivity; Pipe insulation

Thermal transmission properties; Heat flow meter 


\section{SOUTHWEST RESEARCH INSTITUTE \\ DEPARTMENT OF FIRE TECHNOLOGY \\ 6220 Cullebra Road, San Antonio, TX 78238 \\ Carl A. Hafer \\ Phone: 512-684-5111}

Accreditation Renewal Date: January 1, 1986

$\begin{array}{lll}\text { NVLAP Code } & \text { Designation } & \text { Short Title } \\ \text { 03/F01 } & \text { ASTM E84 } & \text { Surface Flammability (Carpet) } \\ \text { 03/F02 } & \text { UL 992 } & \text { Surface Flammability } \\ \text { 03/F03 } & \text { DoC FF 1-70 } & \text { Methenamine Pill Test } \\ \text { 03/F04 } & \text { ASTM E648 } & \text { Radiant Panel (Carpet) }\end{array}$

NVLAP LAB CODE 0115

\section{FACTORY MUTUAL RESEARCH CORPORATION \\ 1151 Boston-Providence Turnpike, Norwood, MA 02062 \\ William F. Maroni Phone: 617-762-4300}

Accreditation Renewal Date: January 1, 1986

\begin{tabular}{|c|c|c|}
\hline NVLAP Code & Designation & Short Title \\
\hline $01 / \mathrm{C} 02$ & $\begin{array}{l}\mathrm{HH}-\mathrm{I}-515 \\
\text { (para. } 4.8 .5 \text { in D version, } \\
\text { Amendment } 1 \text { ) }\end{array}$ & Corrosiveness; Cellulosic fiber (loose-fill) \\
\hline $01 / \mathrm{D} 25$ & $\begin{array}{l}\text { HH-I-515 } \\
\text { (para. } 4.8 .3 \text { in D version, } \\
\text { Amendment } 1 \text { ) }\end{array}$ & Moisture absorption; Cellulosic fiber (loose-fill) \\
\hline 01/D26 & $\begin{array}{l}\text { HH-I-515 } \\
\text { (para. } 4.8 .1 \text { in D version, } \\
\text { Amendment } 1 \text { ) }\end{array}$ & Settled density; Cellulosic fiber (loose-fill) \\
\hline $01 / \mathrm{F} 02$ & ASTM E84 & Surface burning characteristics; Building materials \\
\hline 01/F07 & $\begin{array}{l}\mathrm{HH}-\mathrm{I}-515 \\
\text { (para. } 4.8 .7 \text { in } \mathrm{D} \text { version, } \\
\text { Amendment } 1 \text { ) }\end{array}$ & Critical radiant flux; Radiant Panel (cellulosic fiber, loose-fill) \\
\hline 01/F08 & $\begin{array}{l}\mathrm{HH}-\mathrm{I}-515 \\
\text { (para. } 4.8 .8 \text { in } \mathrm{D} \text { version, } \\
\text { Amendment } 1 \text { ) }\end{array}$ & Smoldering combustion; Cellulosic fiber (loose-fill) \\
\hline 03/F01 & ASTM E84 & Surface Flammability (Carpet) \\
\hline 03/F04 & ASTM E648 & Radiant Panel (Carpet) \\
\hline
\end{tabular}

NVLAP LAB CODE 0116

UNDERWRITERS LABORATORIES INC.

333 Pfingsten Road, Northbrook, IL 60062

Steve Mazzoni Phone: 312-272-8800

Accreditation Renewal Date: January 1, 1986

$\begin{array}{ll}\text { NVLAP Code } & \begin{array}{l}\text { Designation } \\ \text { ASTM C739 } \\ \text { (para. } 10.7 \text { in } 80 \text { version) }\end{array} \\ 01 / \mathrm{C} 02 & \begin{array}{l}\text { HH-I-515 } \\ \text { (para. } 4.8 .5 \text { in D version, }\end{array} \\ & \text { Amendment } 1 \text { ) } \\ 01 / \mathrm{D} 01 & \text { ASTM C136 } \\ 01 / \mathrm{D} 02 & \text { ASTM C167 } \\ 01 / \mathrm{D} 03 & \text { ASTM C209 } \\ & \text { (para. } 6 \text { in } 72 \text { version) }\end{array}$

Short Title

Corrosiveness; Cellulosic fiber (loose-fill)

Corrosiveness; Cellulosic fiber (loose-fill)

Sieve or screen analysis

Thickness and density; Blanket and batt

Thickness; Board (cellulosic fiber) 


\begin{tabular}{|c|c|c|}
\hline NVLAP Code & Designation & Short Title \\
\hline 01/D04 & ASTM C209 & Water absorption, 2 hour; \\
\hline 01/D05 & $\begin{array}{l}\text { ASTM C209 } \\
\text { (para. } 13 \text { in } 72 \text { version) } \\
\text { (para. } 100-106 \text { in } 78 \text { version) }\end{array}$ & Water absorption, 24 hour; Board (cellulosic fiber) by D1037 \\
\hline 01/D06 & $\begin{array}{l}\text { ASTM C209 } \\
\text { (para. } 14 \text { in } 72 \text { version) } \\
\text { (para. } 107-110 \text { in } 72 \text { version) }\end{array}$ & Linear expansion; Board (cellulosic fiber) by D1037 \\
\hline 01/D08 & ASTM C302 & Density; Preformed pipe insulation \\
\hline 01/D09 & ASTM C303 & Density; Preformed block insulation \\
\hline $01 / \mathrm{D} 13$ & ASTM C519 & Density; Loose-fill (fibrous) \\
\hline 01/D14 & ASTM C520 & Density; Granular loose-fill \\
\hline 01/D18 & ASTM D1622 & Apparent density; Rigid cellular plastics \\
\hline 01/D24 & $\begin{array}{l}\text { ASTM C739 } \\
\text { (para. } 10.5 \text { in } 80 \text { version) }\end{array}$ & Moisture absorption; Celluiosic fiber (loose-fill) \\
\hline 01/D25 & $\begin{array}{l}\mathrm{HH}-\mathrm{I}-515 \\
\text { (para. } 4.8 .3 \text { in D version, } \\
\text { Amendment } 1 \text { ) }\end{array}$ & Moisture absorption; Cellulosic fiber (loose-fill) \\
\hline 01/D26 & $\begin{array}{l}\text { HH-I-515 } \\
\text { (para. } 4.8 .1 \text { in D version, } \\
\text { Amendment } 1 \text { ) }\end{array}$ & Settled density; Cellulosic fiber (loose-fill) \\
\hline 01/F02 & ASTM E84 & Surface burning characteristics; Building materials \\
\hline 01/F06 & $\begin{array}{l}\text { ASTM C } 739 \\
\text { (para. } 10.4 \text { in } 80 \text { version) }\end{array}$ & Flame resistance permanency; Cellulosic fiber (loose-fill) \\
\hline 01/F07 & $\begin{array}{l}\text { HH-I-515 } \\
\text { (para. } 4.8 .7 \text { in D version, } \\
\text { Amendment } 1 \text { ) }\end{array}$ & Critical radiant flux; Radiant Panel (cellulosic fiber, loose-fill) \\
\hline 01/F08 & $\begin{array}{l}\text { HH-I- } 515 \\
\text { (para. } 4.8 .8 \text { in D version, } \\
\text { Amendment 1) }\end{array}$ & Smoldering combustion; Cellulosic fiber (loose-fill) \\
\hline 01/S02 & ASTM C203 & Breaking load/flexural strength; Preformed block insulation \\
\hline 01/S03 & $\begin{array}{l}\text { ASTM C } 209 \\
\text { (para. } 9 \text { in } 72 \text { version) }\end{array}$ & Transverse strength; Board (cellulosic fiber) \\
\hline 01/S04 & $\begin{array}{l}\text { ASTM C209 } \\
\text { (para. } 10 \text { in } 72 \text { version) }\end{array}$ & Deflection at specified load; Board (cellulosic fiber) \\
\hline 01/S05 & $\begin{array}{l}\text { ASTM C209 } \\
\text { (para. } 11 \text { in } 72 \text { version) }\end{array}$ & Tensile strength; Parallel to surface; Board (cellulosic fiber) \\
\hline 01/S06 & $\begin{array}{l}\text { ASTM C209 } \\
\text { (para. } 12 \text { in } 72 \text { version) }\end{array}$ & Tensile strength; Perpendicular to surface \\
\hline 01/S08 & ASTM C446 & Breaking load/modulus of rupture; Preformed pipe insulation \\
\hline $01 / \mathrm{S} 11$ & ASTM D1621 & Compressive properties; Rigid cellular plastics (proc. A-Crosshead) \\
\hline 01/T06 & ASTM C518 & Thermal transmission properties; Heat flow meter \\
\hline 01/T09 & ASTM C653 & Thermal resistance (Rec. Practice); Blanket (mineral fiber) \\
\hline 01/T10 & ASTM C687 & Thermal resistance (Rec. Practice); Loose-fill (fibrous) \\
\hline 01/V02 & TAPPI T419 & Starch in paper; Qualitative test \\
\hline 01/V03 & ASTM D2020 & Mildew (fungus) resistance; Paper and paperboard \\
\hline 01/V05 & $\begin{array}{l}\text { HH-I- } 515 \\
\text { (para. } 4.8 .6 \text { in D version, } \\
\text { Amendment 1) }\end{array}$ & Fungus; Cellulosic fiber (loose-fill) \\
\hline 01/V06 & $\begin{array}{l}\text { HH-I-515 } \\
\text { (para. } 4.8 .9 \text { in D version, } \\
\text { Amendment 1) }\end{array}$ & Starch; Cellulosic fiber (loose-fill) \\
\hline 03/F01 & ASTM E84 & Surface Flammability (Carpet) \\
\hline 03/F02 & UL 992 & Surface Flammability \\
\hline 03/F03 & DoC FF 1-70 & Methenamine Pill Test \\
\hline 03/F04 & ASTM E648 & Radiant Panel (Carpet) \\
\hline
\end{tabular}


Section of UL 737

NVLAP Code Short Title

PHYSICAL/FIRE TEST GROUP (04/F00)

04/F01

04/F02

04/F04

04/F05

04/F06

04/F07

04/F08

04/F09

04/F10

04/M01

04/M02

04/M03

04/E01

04/E02

04/E03

04/E04

04/E05

04/E06

04/E07

04/E08
Test Installation

Temperature Measurement

Radiant Fire Test

Coal Fire Test

Brand Fire Test

Flash Fire Test

Strength Tests

Stability Test

Glazing Test

MOBILE HOME TEST GROUP (04/M00)

Test Installation

Toxic Gas

Drop Test

ELECTRICAL TEST GROUP (04/E00)

Test Voltages

Temperature Measurements, Electrical Components

Input Test

Temperature Test, Electrical Components

Leakage Current

Dielectric Withstand

Locked Rotor (Stalled Motor) Temperature

Power Cord Strain Relief 5th Edition

Section of UL 1482

2nd Edition

(March 1, 1982)

(January 24, 1983)

$\begin{array}{rr}8 & 8 \\ 9 & 9 \\ 11 & 11 \\ & 14 \\ 12 & 12 \\ 13 & 13 \\ 15 & 16 \\ 16 & 16 \\ 14 & 15\end{array}$

$\begin{array}{ll}17 & 17 \\ 17 & 17 \\ 17 & 17\end{array}$

$\begin{array}{ll}33 & 33 \\ 34 & 34 \\ 35 & 35 \\ 36 & 36 \\ 38 & 38 \\ 37 & 37 \\ 39 & 39 \\ 40 & 40\end{array}$

NVLAP LAB CODE 0117

\section{UNDERWRITERS LABORATORIES INC. SANTA CLARA, CALIFORNIA LABORATORY 1655 Scott Boulevard, Santa Clara, CA 95050 \\ Douglas Anderson \\ Phone: 408-985-2400}

Accreditation Renewal Date: January 1, 1986

\begin{tabular}{|c|c|c|}
\hline NVLAP Code & Designation & Short Title \\
\hline 01/D13 & ASTM C519 & Density; Loose-f \\
\hline 01/D26 & $\begin{array}{l}\mathrm{HH}-\mathrm{I}-515 \\
\text { (para. } 4.8 .1 \text { in } \mathrm{D} \text { version, } \\
\text { Amendment } 1 \text { ) }\end{array}$ & Settled density; \\
\hline 01/F02 & ASTM E84 & Surface burning \\
\hline 01/F07 & $\begin{array}{l}\text { HH-I-515 } \\
\text { (para. } 4.8 .7 \text { in D version, } \\
\text { Amendment 1) }\end{array}$ & Critical radiant \\
\hline 01/F08 & $\begin{array}{l}\text { HH-I-515 } \\
\text { (para. } 4.8 .8 \text { in D version, } \\
\text { Amendment } 1 \text { ) }\end{array}$ & Smoldering col \\
\hline NVLAP Code & \multicolumn{2}{|c|}{$\begin{array}{l}\text { Short Title } \\
\text { PHYSICAL/FIRE TEST GROUP (04/F00) }\end{array}$} \\
\hline 04/F01 & \multicolumn{2}{|c|}{ Test Installation } \\
\hline 04/F02 & \multicolumn{2}{|l|}{ Temperature Measurement } \\
\hline 04/F04 & \multicolumn{2}{|l|}{ Radiant Fire Test } \\
\hline 04/F05 & \multicolumn{2}{|l|}{ Coal Fire Test } \\
\hline 04/F06 & \multicolumn{2}{|l|}{ Brand Fire Test } \\
\hline
\end{tabular}

Section of UL 737 5th Edition (March 1, 1982)
Section of UL 1482 2nd Edition (January 24, 1983) 


$\begin{array}{ll}\text { NVLAP Code } & \text { Short Title } \\ \text { 04/F07 } & \text { Flash Fire Test } \\ \text { 04/F08 } & \text { Strength Tests } \\ \text { 04/F09 } & \text { Stability Test } \\ \text { 04/F10 } & \text { Glazing Test }\end{array}$

MOBILE HOME TEST GROUP (04/M00)

04/M01

04/M02

04/M03

04/E01

04/E02

$04 / \mathrm{E} 03$

04/E04

04/E05

04/E06

04/E07

04/E08

Drop Test

ELECTRICAL TEST GROUP (04/E00)

Test Voltages

Input Test

Temperature Test, Electrical Components

Leakage Current

Dielectric Withstand

Locked Rotor (Stalled Motor) Temperature

Power Cord Strain Relief
Section of UL 737

5th Edition

(March 1, 1982)

Temperature Measurements, Electrical Components
13

15

16

14

17

17

17

Section of UL 1482

2nd Edition

(January 24, 1983)

13

16

16

15

17

17

17

$33 \quad 33$

$34 \quad 34$

$35 \quad 35$

$36 \quad 36$

$38 \quad 38$

$37-37$

$39-39$

$40-40$

NVLAP LAB CODE 0119

INTEST LABORATORIES, INC.

2820 Anthony Lane South, Minneapolis, MN 55418

Donald L. Valsvik Phone: 612-781-2603

Accreditation Renewal Date: January 1, 1986

$\begin{array}{ll}\text { NVLAP Code } & \text { Designation } \\ \text { 08/P02 } & \text { ASTM C384-77(84) } \\ \text { 08/P03 } & \text { ASTM C423-84a } \\ \text { 08/P06 } & \text { ASTM E90-83 } \\ \text { 08/P11 } & \text { ANSI S1.31-80 } \\ & \\ 08 / \text { E04 } & \text { ANSI S3.19-75 } \\ 08 / \text { E13 } & \text { SAE J192a-75 } \\ \text { 08/E14 } & \text { SAE J1161-76 } \\ 08 / \text { E21 } & \text { AMA-1-11-67 }\end{array}$

Short Title

Impedance and Absorption of Acoustical Materials

Sound Absorption and Sound Absorption Coefficients

Airborne Sound Transmission Loss of Building Partitions

Sound Power Levels, Broad-Band (direct method only)

Noise Sources in Reverberation Rooms (direct method only) $(100-10,000 \mathrm{~Hz})$

Noise Protection, Hearing Protectors and Earmuffs

Exterior Sound Level of Snowmobiles

Sound Level Measurement Procedure for Snow Vehicles

Ceiling Sound Transmission Test by Two-Room Method

NVLAP LAB CODE 0120

COMMERCIAL TESTING COMPANY

1215 South Hamilton Street, P.O. Box 985, Dalton, GA 30720

Jonathan Jackson Phone: 404-278-3935

Accreditation Renewal Date: January 1, 1986

\begin{tabular}{|c|c|c|}
\hline NVLAP Code & Designation & Short Title \\
\hline $01 / \mathrm{C} 02$ & $\begin{array}{l}\text { HH-I-515 } \\
\text { (para. } 4.8 .5 \text { in D version, } \\
\text { Amendment } 1 \text { ) }\end{array}$ & Corrosiveness; Cellulosic fiber (loose-fill) \\
\hline 01/D25 & $\begin{array}{l}\text { HH-I-515 } \\
\text { (para. } 4.8 .3 \text { in D version, } \\
\text { Amendment } 1 \text { ) }\end{array}$ & Moisture absorption; Cellulosic fiber (loose-fill) \\
\hline 01/D26 & $\begin{array}{l}\mathrm{HH}-\mathrm{I}-515 \\
\text { (para. } 4.8 .1 \text { in D version, } \\
\text { Amendment 1) }\end{array}$ & Settled density; Cellulosic fiber (loose-fill) \\
\hline
\end{tabular}




$\begin{array}{lll}\begin{array}{l}\text { NVLAP Code } \\ \text { 01/F07 }\end{array} & \begin{array}{l}\text { Designation } \\ \text { HH-I-515 } \\ \text { (para. 4.8.7 in D version, }\end{array} & \begin{array}{l}\text { Short Title } \\ \text { Critical radiant flux; Radiant Panel (cellulosic fiber, loose-fill) }\end{array} \\ \text { Amendment 1) } \\ \text { 01/F08 } & \begin{array}{l}\text { HH-I-515 } \\ \text { (para. 4.8.8 in D version, }\end{array} & \text { Smoldering combustion; Cellulosic fiber (loose-fill) } \\ & \text { Amendment 1) } & \\ \text { 01/T06 } & \text { ASTM C518 } & \text { Thermal transmission properties; Heat flow meter } \\ \text { 03/C01 } & \text { AATCC 16E } & \text { Colorfastness to Light (Xenon Arc) } \\ \text { 03/C02 } & \text { AATCC 8 } & \text { Colorfastness to Crocking } \\ \text { 03/D01 } & \text { ASTM D418 } & \text { Pile Yarn Floor Covering Construction } \\ & & \text { Pile Weight - Uncoated (Section 8) } \\ & & \text { Pile Weight - Coated (Section 9) } \\ & & \text { Pile Thickness - (Sections 10 \& 11) } \\ \text { Tuft Height - (Section 13) } \\ \text { 03/D02 } & \text { Shrinkage } \\ \text { 03/S01 } & \text { ASTM D1335 } & \text { Tuft Bind of Floor Coverings } \\ & \text { Federal Test Method } & \\ & \text { Standard 191-5100 } & \text { Textile Test Method - Breaking Strength } \\ & \text { Textile Test Method - Delamination } \\ \text { 03/F01 } & \text { ASTM E84 } & \text { Surface Flammability (Carpet) } \\ \text { 03/F03 } & \text { DoC FF 1-70 } & \text { Methenamine Pill Test } \\ \text { 03/F04 } & \text { ASTM E648 } & \text { Radiant Panel (Carpet) } \\ \text { 03/B02 } & \text { UM 44C } & \text { Addenda 2 and 3 Attached Cushion Tests }\end{array}$

NVLAP LAB CODE 0121

\title{
SPARRELL ENGINEERING RESEARCH CORPORATION \\ Bristol Road, P.O. Box 130, Damariscotta, ME 04543 \\ James K. Sparrell Phone: 207-563-3224
}

Accreditation Renewal Date: January 1, 1986

$\begin{array}{ll}\text { NVLAP Code } & \text { Designation } \\ 01 / \mathrm{T} 01 & \text { ASTM C177 } \\ 01 / \text { T04 } & \text { ASTM C236 } \\ 01 / \text { T06 } & \text { ASTM C518 }\end{array}$

\begin{abstract}
Short Title
Thermal transmission properties; Low-temperature guarded hot plate

Thermal conductance; Guarded hot box

Thermal transmission properties; Heat flow meter
\end{abstract}

NVLAP LAB CODE 0122

TECHNICAL MICRONICS CONTROL, INC.

210 Wynn Drive, P.O. Box 1330, Huntsville, AL 35807

Otis Cauthen Phone: 205-837-4430

Accreditation Renewal Date: January 1, 1986

\begin{tabular}{|c|c|}
\hline NVLAP Code & Designation \\
\hline $01 / \mathrm{C} 02$ & $\begin{array}{l}\text { HH-I-515 } \\
\text { (para. } 4.8 .5 \text { in D version, } \\
\text { Amendment } 1 \text { ) }\end{array}$ \\
\hline 01/D25 & $\begin{array}{l}\text { HH-I-515 } \\
\text { (para. } 4.8 .3 \text { in D version, } \\
\text { Amendment } 1 \text { ) }\end{array}$ \\
\hline 01/D26 & $\begin{array}{l}\text { HH-I-515 } \\
\text { (para. } 4.8 .1 \text { in D version, } \\
\text { Amendment } 1 \text { ) }\end{array}$ \\
\hline 01/F07 & $\begin{array}{l}\text { HH-I-515 } \\
\text { (para. } 4.8 .7 \text { in D version, } \\
\text { Amendment } 1 \text { ) }\end{array}$ \\
\hline
\end{tabular}

Short Title

Corrosiveness; Cellulosic fiber (loose-fill)

Moisture absorption; Cellulosic fiber (loose-fill)

Settled density; Cellulosic fiber (loose-fill)

Critical radiant flux; Radiant Panel (cellulosic fiber, loose-fill) 


$\begin{array}{ll}\text { NVLAP Code } & \text { Designation } \\ 01 / \text { F08 } & \text { HH-I-515 } \\ & \text { (para. 4.8.8 in D version, } \\ & \text { Amendment 1) } \\ \text { 01/T06 } & \text { ASTM C518 } \\ 01 / \text { V06 } & \text { HH-I-515 } \\ & \text { (para. 4.8.9 in D version, } \\ & \text { Amendment 1) }\end{array}$

\section{Short Title}

Smoldering combustion; Cellulosic fiber (loose-fill)

Thermal transmission properties; Heat flow meter

Starch; Cellulosic fiber (loose-fill)

\section{MANVILLE CORPORATION, $\mathbb{R}$ \& D CENTER \\ P.O. Box 5108, Denver, CO 80217 \\ Joseph P. Ferraro \\ Phone: 303-978-5553}

Accreditation Renewal Date: January 1, 1986

\begin{tabular}{|c|c|}
\hline NVLAP Code & Designation \\
\hline 01/D02 & ASTM C167 \\
\hline 01/D03 & $\begin{array}{l}\text { ASTM C } 209 \\
\text { (para. } 6 \text { in } 72 \text { version) }\end{array}$ \\
\hline 01/D04 & ASTM C209 \\
\hline 01/D05 & $\begin{array}{l}\text { ASTM C209 } \\
\text { (para. } 13 \text { in } 72 \text { version) } \\
\text { (para. } 100-106 \text { in } 78 \text { version) }\end{array}$ \\
\hline 01/D06 & $\begin{array}{l}\text { ASTM C209 } \\
\text { (para. } 14 \text { in } 72 \text { version) } \\
\text { (para. } 107-110 \text { in } 72 \text { version) }\end{array}$ \\
\hline 01/D08 & ASTM C302 \\
\hline 01/D09 & ASTM C303 \\
\hline $01 / D 11$ & ASTM C356 \\
\hline $01 / \mathrm{D} 12$ & ASTM C411 \\
\hline $01 / \mathrm{D} 13$ & ASTM C519 \\
\hline 01/F01 & TAPPI T461 \\
\hline 01/F02 & ASTM E84 \\
\hline $01 / F 05$ & ASTM E136 \\
\hline $01 / \mathrm{S} 01$ & ASTM C165 \\
\hline 01/S02 & ASTM C203 \\
\hline $01 / \mathrm{S} 03$ & $\begin{array}{l}\text { ASTM C209 } \\
\text { (para. } 9 \text { in } 72 \text { version) }\end{array}$ \\
\hline 01/S04 & $\begin{array}{l}\text { ASTM C209 } \\
\text { (para. } 10 \text { in } 72 \text { version) }\end{array}$ \\
\hline $01 / \mathrm{S} 05$ & $\begin{array}{l}\text { ASTM C } 209 \\
\text { (para. } 11 \text { in } 72 \text { version) }\end{array}$ \\
\hline $01 / \mathrm{S} 06$ & $\begin{array}{l}\text { ASTM C209 } \\
\text { (para. } 12 \text { in } 72 \text { version) }\end{array}$ \\
\hline $01 / \mathrm{S} 08$ & ASTM C446 \\
\hline $01 / \mathrm{S} 09$ & ASTM D781 \\
\hline $01 / \mathrm{S} 10$ & ASTM D828 \\
\hline 01/T01 & ASTM C177 \\
\hline 01/T04 & ASTM C236 \\
\hline 01/T05 & ASTM C335 \\
\hline 01/T06 & ASTM C518 \\
\hline 01/T09 & ASTM C653 \\
\hline $01 / \mathrm{T} 10$ & ASTM C687 \\
\hline 01/V04 & ASTM E96 \\
\hline 08/P02 & ASTM C384-77(84) \\
\hline
\end{tabular}

\section{Short Title}

Thickness and density; Blanket and batt

Thickness; Board (cellulosic fiber)

Water absorption, 2 hour;

Water absorption, 24 hour; Board (cellulosic fiber) by D1037

Linear expansion; Board (cellulosic fiber) by D1037

Density; Preformed pipe insulation

Density; Preformed block insulation

Linear shrinkage; Soaking heat; Preformed high temperature insulation

Hot-surface performance; High temperature insulation

Density; Loose-fill (fibrous)

Flame Resistance; Paper and paperboard

Surface burning characteristics; Building materials

Behavior of Materials in a Vertical Tube Furnace

Compressive properties; Thermal insulation (proc. A)

Breaking load/flexural strength; Preformed block insulation

Transverse strength; Board (cellulosic fiber)

Deflection at specified load; Board (cellulosic fiber)

Tensile strength; Parallel to surface; Board (cellulosic fiber)

Tensile strength; Perpendicular to surface

Breaking load/modulus of rupture; Preformed pipe insulation

Puncture test; Paperboard and fiberboard

Tensile breaking strength; Paper and paperboard

Thermal transmission properties; Low-temperature guarded hot plate

Thermal conductance; Guarded hot box

Thermal conductivity; Pipe insulation

Thermal transmission properties; Heat flow meter

Thermal resistance (Rec. Practice); Blanket (mineral fiber)

Thermal resistance (Rec. Practice); Loose-fill (fibrous)

Water vapor transmission; Thin sheets (proc. A)

Impedance and Absorption of Acoustical Materials 
NVLAP Code

08/P03

08/P04

Designation

ASTM C423-84a

ASTM C522-80

08/P06
Short Title

Sound Absorption and Sound Absorption Coefficients

Airflow Resistance of Acoustical Materials

Airborne Sound Transmission Loss of Building Partitions

NVLAP LAB CODE 0124

\section{OWENS-CORNING FIBERGLAS CORPORATION PLANT LABORATORY}

Box 89, 960 Central Expressway, Santa Clara, CA 95052

J.P. Tetreault Phone: 408-727-3535

Accreditation Renewal Date: January 1, 1986

$\begin{array}{lll}\text { NVLAP Code } & \text { Designation } & \text { Short Title } \\ \text { 01/D02 } & \text { ASTM C167 } & \text { Thickness and density; Blanket and batt } \\ \text { 01/D09 } & \text { ASTM C303 } & \text { Density; Preformed block insulation } \\ \text { 01/T06 } & \text { ASTM C518 } & \text { Thermal transmission properties; Heat flow meter }\end{array}$

NVLAP LAB CODE 0125

\section{OWENS-CORNING FIBERGLAS CORPORATION PLANT LABORATORY \\ 700 McLaren Road, Fairburn, GA 30213 \\ Larry Maynard \\ Phone: 404-969-2915}

Accreditation Renewal Date: January 1, 1986

$\begin{array}{lll}\text { NVLAP Code } & \text { Designation } & \text { Short Title } \\ 01 / \text { T06 } & \text { ASTM C518 } & \text { Thermal transmission properties; Heat flow meter }\end{array}$

NVLAP LAB CODE 0126

\section{OWENS-CORNING FIBERGLAS CORPORATION PLANT LABORATORY \\ 300 Sunshine Road, Kansas City, KS 66115 \\ C.E. Husmann Phone: 913-281-2811}

Accreditation Renewal Date: January 1, 1986

$\begin{array}{lll}\text { NVLAP Code } & \text { Designation } & \text { Short Title } \\ \text { 01/D02 } & \text { ASTM C167 } & \text { Thickness and density; Blanket and batt } \\ \text { 01/D09 } & \text { ASTM C303 } & \text { Density; Preformed block insulation } \\ \text { 01/T06 } & \text { ASTM C518 } & \text { Thermal transmission properties; Heat flow meter }\end{array}$

NVLAP LAB CODE 0127

\section{OWENS-CORNING FIBERGLAS CORPORATION PLANT LABORATORY}

Box 8, Davis \& Shreeve Roads, Barrington, NJ 08007

Charles Sitka Phone: 609-547-9200

Accreditation Renewal Date: January 1, 1986

$\begin{array}{ll}\text { NVLAP Code } & \text { Designation } \\ 01 / \text { D02 } & \text { ASTM C167 } \\ 01 / \text { D09 } & \text { ASTM C303 } \\ 01 / \text { T06 } & \text { ASTM C518 }\end{array}$

NVLAP Code Designation

01/D09 ASTM C303

ASTM C518 


\section{OWENS-CORNING FIBERGLAS CORPORATION}

PLANT LABORATORY

P.O. Box 89, Delmar, NY 12054

Mark P. Arnold Phone: 518-439-9341

Accreditation Renewal Date: January 1, 1986

NVLAP Code Designation

01/D02 ASTM Cl67

01/T06 ASTM C518
Short Title

Thickness and density; Blanket and batt

Thermal transmission properties; Heat flow meter

NVLAP LAB CODE 0129

\section{OWENS-CORNING FIBERGLAS CORPORATION}

\section{PLANT LABORATORY}

Case Avenue, Newark, $\mathrm{OH} 43055$

P. D. Shull Phone: 614-345-3441

Accreditation Renewal Date: January 1, 1986

$\begin{array}{ll}\text { NVLAP Code } & \text { Designation } \\ \text { 01/D02 } & \text { ASTM C167 } \\ \text { 01/D09 } & \text { ASTM C303 } \\ \text { 01/T06 } & \text { ASTM C518 }\end{array}$

Short Title

Thickness and density; Blanket and batt

Density; Preformed block insulation

Thermal transmission properties; Heat flow meter

NVLAP LAB CODE 0130

\section{OWENS-CORNING FIBERGLAS CORPORATION PLANT LABORATORY}

P.O. Box 837, I-35 East, Waxahachie, TX 75165

Mark Kwasowski Phone: 214937-1340

Accreditation Renewal Date: January 1, 1986

$\begin{array}{ll}\text { NVLAP Code } & \text { Designation } \\ \text { 01/D02 } & \text { ASTM C167 } \\ \text { 01/D09 } & \text { ASTM C303 } \\ \text { 01/T06 } & \text { ASTM C518 }\end{array}$

\author{
Short Title \\ Thickness and density; Blanket and batt \\ Density; Preformed block insulation \\ Thermal transmission properties; Heat flow meter
}

NVLAP LAB CODE 0131

THE H. C. NUTTING COMPANY

4120 Airport Road, P.O. Box C, Cincinnati, OH 45226

James T. Larbes Phone: 513-321-5816

Accreditation Renewal Date: January 1, 1986

$\begin{array}{ll}\text { NVLAP Code } & \text { Designation } \\ \text { 02/M01 } & \text { ASTM C31 } \\ \text { 02/M03 } & \text { ASTM C172 } \\ \text { 02/P01 } & \text { ASTM C143 } \\ \text { 02/W01 } & \text { ASTM C138 } \\ \text { 02/A01 } & \text { ASTM C231 } \\ \text { 02/S01 } & \text { ASTM C39 } \\ \text { 02/A02 } & \text { ASTM C173 }\end{array}$

Short Title

Making and Curing Concrete Test Specimens in the Field

Sampling Fresh Concrete

Slump of Portland Cement Concrete

Unit Weight, Yield, and Air Content (Gravimetric) of Concrete

Air Content of Freshly Mixed Concrete by the Pressure Method

Compressive Strength of Cylindrical Concrete Specimens

Air Content of Freshly Mixed Concrete by the Volumetric Method 
THE WALT KEELER COMPANY, INC.

826 East Lincoln Street, P.O. Box 197, Wichita, KS 67201

Kelly B. Callison Phone: 316-265-0615

Accreditation Renewall Date: January 1, 1986

$\begin{array}{ll}\text { NVLAP Code } & \text { Designation } \\ \text { 02/M01 } & \text { ASTM C31 } \\ \text { 02/M03 } & \text { ASTM C172 } \\ \text { 02/P01 } & \text { ASTM C143 } \\ \text { 02/W01 } & \text { ASTM C138 } \\ \text { 02/A01 } & \text { ASTM C231 } \\ \text { 02/A02 } & \text { ASTM C173 }\end{array}$

Short Title

Making and Curing Concrete Test Specimens in the Field

Sampling Fresh Concrete

Slump of Portland Cement Concrete

Unit Weight, Yield, and Air Content (Gravimetric) of Concrete

Air Content of Freshly Mixed Concrete by the Pressure Method

Air Content of Freshly Mixed Concrete by the Volumetric Method

NVLAP LAB CODE 0135

\section{AGUIRRE ENGINEERS, INC. \\ 13276 East Fremont Place, P.O. Box 3014, Englewood, CO 80155 \\ Jeffrey C. Olson Phone: 303-799-8378}

Accreditation Renewal Date: January 1, 1986

$\begin{array}{ll}\text { NVLAP Code } & \text { Designation } \\ \text { 02/M01 } & \text { ASTM C31 } \\ \text { 02/M03 } & \text { ASTM C172 } \\ \text { 02/P01 } & \text { ASTM C143 } \\ 02 / \mathrm{W} 01 & \text { ASTM C138 } \\ 02 / \mathrm{A01} & \text { ASTM C231 } \\ 02 / \mathrm{S} 01 & \text { ASTM C39 } \\ 02 / \mathrm{A02} & \text { ASTM C173 }\end{array}$

Short Title

Making and Curing Concrete Test Specimens in the Field

Sampling Fresh Concrete

Slump of Portland Cement Concrete

Unit Weight, Yield, and Air Content (Gravimetric) of Concrete

Air Content of Freshly Mixed Concrete by the Pressure Method

Compressive Strength of Cylindrical Concrete Specimens

Air Content of Freshly Mixed Concrete by the Volumetric Method

NVLAP LAB CODE 0136

CONTRACTORS SUPPLY CORPORATION OF WEST VIRGINLA, INC.

P.O. Box 6587, 24th \& Water, Wheeling, WV 26003

Anthony A. Gulo Phone: 304-232-1048

Accreditation Renewal Date: January 1, 1986

$\begin{array}{ll}\text { NVLAP Code } & \text { Designation } \\ \text { 02/M01 } & \text { ASTM C31 } \\ 02 / \mathrm{M} 03 & \text { ASTM C172 } \\ 02 / \mathrm{P} 01 & \text { ASTM C143 } \\ 02 / \mathrm{W} 01 & \text { ASTM C138 } \\ 02 / \mathrm{A} 01 & \text { ASTM C231 } \\ 02 / \mathrm{S} 01 & \text { ASTM C39 }\end{array}$

Short Title

Making and Curing Concrete Test Specimens in the Field

Sampling Fresh Concrete

Slump of Portland Cement Concrete

Unit Weight, Yield, and Air Content (Gravimetric) of Concrete

Air Content of Freshly Mixed Concrete by the Pressure Method

Compressive Strength of Cylindrical Concrete Specimens

NVLAP LAB CODE 0137

\section{CONSTRUCTION TECHNOLOGY LABORATORIES \\ A DIVISION OF PORTLAND CEMENT ASSOCIATION}

5420 Old Orchard Road, Skokie, IL 60077

Ronald G. Burg Phone: 312-965-7500

Accreditation Renewal Date: January 1, 1986

$\begin{array}{ll}\text { NVLAP Code } & \text { Designation } \\ \text { 02/M01 } & \text { ASTM C31 } \\ \text { 02/M03 } & \text { ASTM C172 }\end{array}$
Short Title

Making and Curing Concrete Test Specimens in the Field

Sampling Fresh Concrete 


$\begin{array}{ll}\text { NVLAP Code } & \text { Designation } \\ \text { 02/P01 } & \text { ASTM C143 } \\ \text { 02/W01 } & \text { ASTM C138 } \\ \text { 02/A01 } & \text { ASTM C231 } \\ \text { 02/S01 } & \text { ASTM C39 } \\ \text { 02/A02 } & \text { ASTM C173 }\end{array}$

Short Title

Slump of Portland Cement Concrete

Unit Weight, Yield, and Air Content (Gravimetric) of Concrete

Air Content of Freshly Mixed Concrete by the Pressure Method

Compressive Strength of Cylindrical Concrete Specimens

Air Content of Freshly Mixed Concrete by the Volumetric Method

NVLAP LAB CODE 0139

AMERICAN CARPET LABORATORIES, INC.

111 West Nashville Street, P.O. Box 357, Ringgold, GA 30736

Michael D. Connell Phone: 404-935-5672

Accreditation Renewal Date: January 1, 1986

$\begin{array}{ll}\text { NVLAP Code } & \begin{array}{l}\text { Designation } \\ \text { 03/C01 }\end{array} \\ \text { AATCC 16E } \\ \text { 03/C02 } & \text { AATCC 8 } \\ \text { 03/D01 } & \text { ASTM D418 } \\ & \\ & \\ & \\ \text { 03/D02 } & \text { DDD-C-95A } \\ \text { 03/S01 } & \text { ASTM D1335 } \\ & \text { Federal Test Method } \\ & \text { Standard 191-5100 } \\ & \text { 191-5950 } \\ \text { 03/F03 } & \text { DoC FF 1-70 } \\ \text { 03/B02 } & \text { UM 44C }\end{array}$

Short Title
Colorfastness to Light (Xenon Arc)
Colorfastness to Crocking
Pile Yarn Floor Covering Construction
Pile Weight - Uncoated (Section 8)
Pile Weight - Coated (Section 9)
Pile Thickness - (Sections 10 \& 11)
Tuft Height - (Section 13)
Shrinkage
Tuft Bind of Floor Coverings
Textile Test Method - Breaking Strength
Textile Test Method - Delamination
Methenamine Pill Test
Addenda 2 and 3 Attached Cushion Tests

NVLAP LAB CODE 0141

\section{GENSTAR STONE PRODUCTS COMPANY \\ WHITE MARSH TECHNICAL CENTER \\ 10300 Pulaski Highway, White Marsh, MD 21162 \\ Robert L. Chester \\ Phone: 301-628-4000}

Accreditation Renewal Date: January 1, 1986

$\begin{array}{ll}\text { NVLAP Code } & \text { Designation } \\ \text { 02/M01 } & \text { ASTM C31 } \\ \text { 02/M03 } & \text { ASTM C172 } \\ \text { 02/P01 } & \text { ASTM C143 } \\ \text { 02/W01 } & \text { ASTM C138 } \\ \text { 02/A01 } & \text { ASTM C231 } \\ \text { 02/S01 } & \text { ASTM C39 } \\ 02 / \mathrm{A} 02 & \text { ASTM C173 }\end{array}$

Short Title
Making and Curing Concrete Test Specimens in the Field
Sampling Fresh Concrete
Slump of Portland Cement Concrete
Unit Weight, Yield, and Air Content (Gravimetric) of Concrete
Air Content of Freshly Mixed Concrete by the Pressure Method
Compressive Strength of Cylindrical Concrete Specimens
Air Content of Freshly Mixed Concrete by the Volumetric Method

NVLAP LAB CODE 0142

\section{GEOSCIENCE LTD.}

410 South Cedros Avenue, Solana Beach, CA 92075

Heinz F. Poppendiek Phone: 619-755-9396

Accreditation Renewal Date: January 1, 1986

$\begin{array}{ll}\text { NVLAP Code } & \text { Designation } \\ \text { 01/D08 } & \text { ASTM C302 } \\ \text { 01/F05 } & \text { ASTM E136 }\end{array}$

Short Title

Density; Preformed pipe insulation

Behavior of Materials in a Vertical Tube Furnace 
NVLAP Code Designation

01/T01 ASTM C177

01/T04 ASTM C236
Short Title

Thermal transmission properties; Low-temperature guarded hot plate Thermal conductance; Guarded hot box

NVLAP LAB CODE 0143

\author{
KELSO INDUSTRIES, INC. \\ QUALITY CONTROL LABORATORY \\ P.O. Box 659, Galveston, TX 77553 \\ Chris G. Slate Phone: 713-744-5341
}

Accreditation Renewal Date: January 1, 1986

$\begin{array}{ll}\text { NVLAP Code } & \text { Designation } \\ \text { 02/M01 } & \text { ASTM C31 } \\ \text { 02/M03 } & \text { ASTM C172 } \\ \text { 02/P01 } & \text { ASTM C143 } \\ \text { 02/W01 } & \text { ASTM C138 } \\ \text { 02/A01 } & \text { ASTM C231 } \\ \text { 02/S01 } & \text { ASTM C39 } \\ 02 / \mathrm{A} 02 & \text { ASTM C173 }\end{array}$

\section{Short Title}

Making and Curing Concrete Test Specimens in the Field

Sampling Fresh Concrete

Slump of Portland Cement Concrete

Unit Weight, Yield, and Air Content (Gravimetric) of Concrete

Air Content of Freshly Mixed Concrete by the Pressure Method

Compressive Strength of Cylindrical Concrete Specimens

Air Content of Freshly Mixed Concrete by the Volumetric Method

NVLAP LAB CODE 0146

\title{
AMERICAN TESTING LABORATORIES, INC. \\ Box 4014, 784 Flory Mill Road, Lancaster, PA 17604 \\ John S. Kassees Phone: 717-569-0488
}

Accreditation Renewal Date: April 1, 1985

$\begin{array}{ll}\text { NVLAP Code } & \text { Designation } \\ \text { 02/M01 } & \text { ASTM C31 } \\ \text { 02/M03 } & \text { ASTM C172 } \\ \text { 02/P01 } & \text { ASTM C } 143 \\ \text { 02/W01 } & \text { ASTM C138 } \\ \text { 02/A01 } & \text { ASTM C231 } \\ \text { 02/S01 } & \text { ASTM C39 } \\ \text { 02/A02 } & \text { ASTM C173 }\end{array}$

\author{
Short Title \\ Making and Curing Concrete Test Specimens in the Field \\ Sampling Fresh Concrete \\ Slump of Portland Cement Concrete \\ Unit Weight, Yield, and Air Content (Gravimetric) of Concrete \\ Air Content of Freshly Mixed Concrete by the Pressure Method \\ Compressive Strength of Cylindrical Concrete Specimens \\ Air Content of Freshly Mixed Concrete by the Volumetric Method
}

NVLAP LAB CODE 0149

\section{E \& B CARPET MILLS}

1020 Riverbend Drive, P.O. Box 2047, Dalton, GA 30720

Robert H. Davis

Accreditation Renewal Date: January 1, 1986

$\begin{array}{ll}\text { NVLAP Code } & \text { Designation } \\ \text { 03/C01 } & \text { AATCC 16E } \\ \text { 03/C02 } & \text { AATCC 8 } \\ \text { 03/D01 } & \text { ASTM D418 } \\ & \\ & \\ \text { 03/D02 } & \text { DDD-C-95A } \\ \text { 03/S01 } & \text { ASTM D1335 } \\ & \text { Federal Test Method } \\ & \text { Standard 191-5100 } \\ & \text { 191-5950 } \\ \text { 03/F03 } & \text { DoC FF 1-70 }\end{array}$

Short Title

Colorfastness to Light (Xenon Arc)

Colorfastness to Crocking

Pile Yarn Floor Covering Construction

Pile Weight - Uncoated (Section 8)

Pile Weight - Coated (Section 9)

Pile Thickness - (Sections 10 \& 11)

Tuft Height - (Section 13)

Shrinkage

Tuft Bind of Floor Coverings

Textile Test Method - Breaking Strength

Textile Test Method - Delamination

Methenamine Pill Test 


\section{HARDWOOD PLYWOOD MANUFACTURERS ASSOCIATION}

P.O. Box 2789, 1825 Faraday Drive, Reston, VA 22090

William J. Groah Phone: 703-435-2900

Accreditation Renewal Date: January 1, 1986

$\begin{array}{lll}\text { NVLAP Code } & \text { Designation } & \text { Short Title } \\ \text { 01/F02 } & \text { ASTM E84 } & \text { Surface burning characteristics; Building materials } \\ \text { 01/F07 } & \text { HH-I-515 } & \text { Critical radiant flux; Radiant Panel (cellulosic fiber, loose-fill) } \\ & \text { (para. 4.8.7 in D version, } & \\ & \text { Amendment 1) } & \\ \text { 03/F01 } & \text { ASTM E84 } & \text { Surface Flammability (Carpet) } \\ \text { 03/F04 } & \text { ASTM E648 } & \text { Radiant Panel (Carpet) }\end{array}$

NVLAP LAB CODE 0154

\section{THE ARUNDEL CORPORATION GREENSPRING LABORATORY \\ 6806 Greenspring Avenue, Baltimore, MD 21209 \\ David Wherley Phone: 301-296-6400}

Accreditation Renewal Date: January 1, 1986

$\begin{array}{ll}\text { NVLAP Code } & \text { Designation } \\ \text { 02/M01 } & \text { ASTM C31 } \\ \text { 02/M03 } & \text { ASTM C172 } \\ \text { 02/P01 } & \text { ASTM C143 } \\ \text { 02/W01 } & \text { ASTM C138 } \\ \text { 02/A01 } & \text { ASTM C231 } \\ \text { 02/S01 } & \text { ASTM C39 } \\ \text { 02/A02 } & \text { ASTM C173 }\end{array}$

Short Title

Making and Curing Concrete Test Specimens in the Field

Sampling Fresh Concrete

Slump of Portland Cement Concrete

Unit Weight, Yield, and Air Content (Gravimetric) of Concrete

Air Content of Freshly Mixed Concrete by the Pressure Method

Compressive Strength of Cylindrical Concrete Specimens

Air Content of Freshly Mixed Concrete by the Volumetric Method

NVLAP LAB CODE 0156

\section{BIGELOW-SANFORD, INC.} GEORGIA RUG MILL

Lyerly Street, Summerville, GA 30747

Van A. Pullen Phone: 404-857-2421

Accreditation Renewal Date: January 1, 1986

$\begin{array}{ll}\text { NVLAP Code } & \begin{array}{l}\text { Designation } \\ \text { 03/C01 }\end{array} \\ \text { 03/C02 } & \text { AATCC 16E } \\ \text { 03/D01 } & \text { ASTM D418 } \\ & \\ & \\ & \\ \text { 03/D02 } & \text { DDD-C-95A } \\ \text { 03/S01 } & \text { ASTM D1335 } \\ & \text { Federal Test Method } \\ & \text { Standard 191-5100 } \\ & \text { 191-5950 } \\ \text { 03/F03 } & \text { DoC FF 1-70 } \\ \text { 03/B01 } & \text { UM 44C }\end{array}$

Short Title

Colorfastness to Light (Xenon Arc)

Colorfastness to Crocking

Pile Yarn Floor Covering Construction

Pile Weight - Uncoated (Section 8)

Pile Weight - Coated (Section 9)

Pile Thickness - (Sections 10 \& 11)

Tuft Height - (Section 13)

Shrinkage

Tuft Bind of Floor Coverings

Textile Test Method - Breaking Strength

Textile Test Method - Delamination

Methenamine Pill Test

Addendum 3 Attached Cushion Tests 
CHISHOLM TRAIL TESTING AND ENGINEERING COMPANY, INC.

302 South Miller Street, Decatur, TX 76234

James F. Rosendahl Phone: 817-627-5216

Accreditation Renewal Date: January 1, 1986

$\begin{array}{ll}\text { NVLAP Code } & \text { Designation } \\ \text { 03/C01 } & \text { AATCC 16E } \\ \text { 03/C02 } & \text { AATCC } 8 \\ \text { 03/D01 } & \text { ASTM D418 } \\ & \\ & \\ \text { 03/D02 } & \text { DDD-C-95A } \\ \text { 03/S01 } & \text { ASTM D1335 } \\ & \text { Federal Test Method } \\ & \text { Standard 191-5100 } \\ & \text { 191-5950 } \\ \text { 03/F03 } & \text { DoC FF 1-70 }\end{array}$

Short Title

Colorfastness to Light (Xenon Arc)

Colorfastness to Crocking

Pile Yarn Floor Covering Construction

Pile Weight - Uncoated (Section 8)

Pile Weight - Coated (Section 9)

Pile Thickness - (Sections 10 \& 11)

Tuft Height - (Section 13)

Shrinkage

Tuft Bind of Floor Coverings

Textile Test Method - Breaking Strength

Textile Test Method - Delamination

Methenamine Pill Test

NVLAP LAB CODE 0163

GALAXY CARPET MILLS, INC. GALAXY TESTING LABORATORY

P.O. Box 800, Industrial Blvd., Chatsworth, GA 30705

Lou Childers Phone: 404-695-9611

Accreditation Renewal Date: January 1, 1986

\begin{tabular}{|c|c|c|}
\hline NVLAP Code & Designation & Short Title \\
\hline $03 / \mathrm{C} 01$ & AATCC $16 \mathrm{E}$ & Colorfastness to Light (Xenon Arc) \\
\hline 03/C02 & AATCC 8 & Colorfastness to Crocking \\
\hline 03/D01 & ASTM D418 & $\begin{array}{l}\text { Pile Yarn Floor Covering Construction } \\
\text { Pile Weight - Uncoated (Section 8) } \\
\text { Pile Weight - Coated (Section 9) } \\
\text { Pile Thickness - (Sections 10 \& 11) } \\
\text { Tuft Height - (Section 13) }\end{array}$ \\
\hline 03/D02 & DDD-C-95A & Shrinkage \\
\hline 03/S01 & $\begin{array}{l}\text { ASTM D1335 } \\
\text { Federal Test Method }\end{array}$ & Tuft Bind of Floor Coverings \\
\hline & $\begin{array}{l}\text { Standard 191-5100 } \\
191-5950\end{array}$ & $\begin{array}{l}\text { Textile Test Method - Breaking Strength } \\
\text { Textile Test Method - Delamination }\end{array}$ \\
\hline 03/F03 & DoC FF 1-70 & Methenamine Pill Test \\
\hline 03/B02 & UM 44C & Addenda 2 and 3 Attached Cushion Tests \\
\hline
\end{tabular}

NVLAP LAB CODE 0166

INDEPENDENT TEXTILE TESTING SERVICE, INC.

P.O. Box 1948, 1503 Murray Avenue, Dalton, GA 30720

Cornelius C. Setter

Phone: 404-278-3013

Accreditation Renewal Date: January 1, 1986

$\begin{array}{ll}\text { NVLAP Code } & \text { Designation } \\ 03 / \mathrm{C} 01 & \text { AATCC } 16 \mathrm{E} \\ 03 / \mathrm{C} 02 & \text { AATCC } 8\end{array}$

Short Title

Colorfastness to Light (Xenon Arc)

Colorfastness to Crocking 


\section{NVLAP Code Designation \\ 03/D01 ASTM D418}

03/D02

03/S01

$03 / \mathrm{E} 01$

03/F03

$03 / \mathrm{F} 04$

03/B02
DDD-C-95A

ASTM D1335

Federal Test Method

Standard 191-5100

191-5950

AATCC 134/CRI 102

DoC FF 1-70

ASTM E648

UM 44C
Short Title

Pile Yarn Floor Covering Construction

Pile Weight - Uncoated (Section 8)

Pile Weight - Coated (Section 9)

Pile Thickness - (Sections 10 \& 11)

Tuft Height - (Section 13)

Shrinkage

Tuft Bind of Floor Coverings

Textile Test Method - Breaking Strength

Textile Test Method - Delamination

Electrostatic Propensity of Carpets

Methenamine Pill Test

Radiant Panel (Carpet)

Addenda 2 and 3 Attached Cushion Tests

NVLAP LAB CODE 0173

\section{STS CONSULTANTS, LTD. \\ RALEIGH NC OFFICE}

P.O. Box 12015, Research Triangle Park, NC 27709

Wayne V. Wilkinson Phone: 919-787-5124

Accreditation Renewal Date: April 1, 1985

$\begin{array}{ll}\text { NVLAP Code } & \text { Designation } \\ \text { 02/M01 } & \text { ASTM C31 } \\ \text { 02/M03 } & \text { ASTM C172 } \\ \text { 02/P01 } & \text { ASTM C143 } \\ \text { 02/W01 } & \text { ASTM C138 } \\ \text { 02/A01 } & \text { ASTM C231 } \\ \text { 02/S01 } & \text { ASTM C39 } \\ \text { 02/A02 } & \text { ASTM C173 }\end{array}$

Short Title

Making and Curing Concrete Test Specimens in the Field

Sampling Fresh Concrete

Slump of Portland Cement Concrete

Unit Weight, Yield, and Air Content (Gravimetric) of Concrete

Air Content of Freshly Mixed Concrete by the Pressure Method

Compressive Strength of Cylindrical Concrete Specimens

Air Content of Freshly Mixed Concrete by the Volumetric Method

NVLAP LAB CODE 0175

\section{THE UPJOHN COMPANY \\ DONALD S. GILMORE RESEARCH LABORATORIES \\ 410 Sackett Point Road, North Haven, CT 06473 \\ Herbert G. Nadeau Phone: 203-281-2762}

Accreditation Renewal Date: October 1, 1985

$\begin{array}{ll}\text { NVLAP Code } & \text { Designation } \\ 01 / \text { D21 } & \text { ASTM D2126 } \\ 01 / \text { D28 } & \text { ASTM D2126 } \\ 01 / \text { T06 } & \text { ASTM C518 }\end{array}$

Short Title

Response to thermal and humid aging (proc. E); Rigid cellular plastics Response to thermal and humid aging (proc. G); Rigid cellular plastics Thermal transmission properties; Heat flow meter 


\section{W. R. GRACE \& COMPANY CONSTRUCTION PRODUCTS DIVISION \\ 62 Whittemore Avenue, Cambridge, MA 02140 \\ Stephen A. Valle Phone: 617-876-1400}

Accreditation Renewa! Date: January 1, 1986

$\begin{array}{ll}\text { NVLAP Code } & \text { Designation } \\ \text { 02/M01 } & \text { ASTM C31 } \\ \text { 02/M03 } & \text { ASTM C172 } \\ \text { 02/P01 } & \text { ASTM C143 } \\ \text { 02/W01 } & \text { ASTM C138 } \\ \text { 02/A01 } & \text { ASTM C231 } \\ \text { 02/S01 } & \text { ASTM C39 } \\ \text { 02/A02 } & \text { ASTM C173 }\end{array}$

Short Title

Making and Curing Concrete Test Specimens in the Field

Sampling Fresh Concrete

Slump of Portland Cement Concrete

Unit Weight, Yield, and Air Content (Gravimetric) of Concrete

Air Content of Freshly Mixed Concrete by the Pressure Method Compressive Strength of Cylindrical Concrete Specimens

Air Content of Freshly Mixed Concrete by the Volumetric Method

NVLAP LAB CODE 0177

\section{ATLANTIC TESTING LABS, LTD. CICERO DIVISION}

P.O. Box 356, Rte 31 at Rte 81, Cicero, NY 13039

Robert van der Horst

Phone: 315-699-5281

Accreditation Renewal Date: April 1, 1985

$\begin{array}{ll}\text { NVLAP Code } & \text { Designation } \\ \text { 02/M01 } & \text { ASTM C31 } \\ 02 / \mathrm{M} 03 & \text { ASTM C172 } \\ 02 / \mathrm{P} 01 & \text { ASTM C143 } \\ \text { 02/W01 } & \text { ASTM C138 } \\ \text { 02/A01 } & \text { ASTM C231 } \\ \text { 02/S01 } & \text { ASTM C39 } \\ \text { 02/A02 } & \text { ASTM C173 }\end{array}$

Short Title
Making and Curing Concrete Test Specimens in the Field
Sampling Fresh Concrete
Slump of Portland Cement Concrete
Unit Weight, Yield, and Air Content (Gravimetric) of Concrete
Air Content of Freshly Mixed Concrete by the Pressure Method
Compressive Strength of Cylindrical Concrete Specimens
Air Content of Freshly Mixed Concrete by the Volumetric Method

NVLAP LAB CODE 0178

BIGELOW-SANFORD, INC. TECHNICAL SERVICES

P.O. Box 3089, Greenville, SC 29602

Hamir D. Merchant Phone: 803-299-2630

Accreditation Renewal Date: January 1, 1986

$\begin{array}{ll}\text { NVLAP Code } & \text { Designation } \\ 03 / \mathrm{C} 01 & \text { AATCC } 16 \mathrm{E} \\ 03 / \mathrm{C} 02 & \text { AATCC } 8 \\ 03 / \mathrm{D} 01 & \text { ASTM D418 }\end{array}$

Short Title

Colorfastness to Light (Xenon Arc)

Colorfastness to Crocking

Pile Yarn Floor Covering Construction

Pile Weight - Uncoated (Section 8)

Pile Weight - Coated (Section 9)

Pile Thickness - (Sections 10 \& 11)

Tuft Height - (Section 13)

$\begin{array}{ll}\text { 03/D02 } & \text { DDD-C-95A } \\ \text { 03/S01 } & \text { ASTM D1335 } \\ & \text { Federal Test Method } \\ & \text { Standard } 191-5100 \\ & \text { 191-5950 } \\ \text { 03/E01 } & \text { AATCC } 134 / \text { CRI } 102 \\ \text { 03/F03 } & \text { DoC FF } 1-70\end{array}$

Shrinkage

Tuft Bind of Floor Coverings

Textile Test Method - Breaking Strength

Textile Test Method - Delamination

Electrostatic Propensity of Carpets

Methenamine Pill Test 
NVLAP Code

03/F04

03/B01
Designation

ASTM E648

UM 44C
Short Title

Radiant Panel (Carpet)

Addendum 3 Attached Cushion Tests

NVLAP LAB CODE 0183

\title{
A \& H/FLOOD ENGINEERING \\ 4421 Harrison Street, Hillside, IL 60162 \\ Paul E. Flood \\ Phone: 312-449-0500
}

Accreditation Renewal Date: April 1, 1985

NVLAP Code Designation

02/M01 ASTM C31

02/M03 ASTM C172

02/P01 ASTM C143

02/W01 ASTM C138

02/A01 ASTM C231

02/S01 ASTM C39

02/A02 ASTM C173

\author{
Short Title \\ Making and Curing Concrete Test Specimens in the Field \\ Sampling Fresh Concrete \\ Slump of Portland Cement Concrete \\ Unit Weight, Yield, and Air Content (Gravimetric) of Concrete \\ Air Content of Freshly Mixed Concrete by the Pressure Method \\ Compressive Strength of Cylindrical Concrete Specimens \\ Air Content of Freshly Mixed Concrete by the Volumetric Method
}

NVLAP LAB CODE 0187

\author{
C. H. MASLAND AND SONS \\ P.O. Box 40, Carlisle, PA 17013 \\ David A. Boyles Phone: 717-249-1866
}

Accreditation Renewal Date: January 1, 1986

$\begin{array}{ll}\text { NVLAP Code } & \text { Designation } \\ \text { 03/C01 } & \text { AATCC 16E } \\ \text { 03/C02 } & \text { AATCC 8 } \\ \text { 03/D01 } & \text { ASTM D418 } \\ & \\ & \\ \text { 03/D02 } & \text { DDD-C-95A } \\ \text { 03/S01 } & \text { ASTM D1335 } \\ & \text { Federal Test Method } \\ & \text { Standard 191-5100 } \\ & \text { 191-5950 } \\ \text { 03/F03 } & \text { DoC FF 1-70 }\end{array}$

Short Title

Colorfastness to Light (Xenon Arc)

Colorfastness to Crocking

Pile Yarn Floor Covering Construction

Pile Weight - Uncoated (Section 8)

Pile Weight - Coated (Section 9)

Pile Thickness - (Sections 10 \& 11)

Tuft Height - (Section 13)

Shrinkage

Tuft Bind of Floor Coverings

Textile Test Method - Breaking Strength

Textile Test Method - Delamination

Methenamine Pill Test

NVLAP LAB CODE 0188

\section{TWIN CITY TESTING AND ENGINEERING LABORATORY, INC.}

662 Cromwell Avenue, St. Paul, MN 55114

Richard Stehly

Phone: 612-645-3601

Accreditation Renewal Date: January 1, 1986

$\begin{array}{ll}\text { NVLAP Code } & \text { Designation } \\ \text { 01/T04 } & \text { ASTM C236 } \\ \text { 02/M01 } & \text { ASTM C31 } \\ \text { 02/M03 } & \text { ASTM C172 } \\ \text { 02/P01 } & \text { ASTM C143 } \\ \text { 02/W01 } & \text { ASTM C138 } \\ \text { 02/A01 } & \text { ASTM C231 }\end{array}$

Short Title

Thermal conductance; Guarded hot box

Making and Curing Concrete Test Specimens in the Field

Sampling Fresh Concrete

Slump of Portland Cement Concrete

Unit Weight, Yield, and Air Content (Gravimetric) of Concrete

Air Content of Freshly Mixed Concrete by the Pressure Method 


$\begin{array}{ll}\text { NVLAP Code } & \text { Designation } \\ \text { 02/S01 } & \text { ASTM C39 } \\ \text { 02/A02 } & \text { ASTM C173 }\end{array}$

Short Title

Compressive Strength of Cylindrical Concrete Specimens

Air Content of Freshly Mixed Concrete by the Volumetric Method

NVLAP LAB CODE 0189

\author{
CENTRAL READY-MIXED CONCRETE \\ RESEARCH \& TECHNICAL CENTER \\ 4350 South 13th Street, Milwaukee, WI 53221 \\ Christine B. Madderom \\ Phone: 414-282-4200
}

Accreditation Renewal Date: January 1, 1986

$\begin{array}{ll}\text { NVLAP Code } & \text { Designation } \\ \text { 02/M01 } & \text { ASTM C31 } \\ \text { 02/M03 } & \text { ASTM C172 } \\ \text { 02/P01 } & \text { ASTM C143 } \\ \text { 02/W01 } & \text { ASTM C138 } \\ \text { 02/A01 } & \text { ASTM C231 } \\ 02 / \mathrm{S} 01 & \text { ASTM C39 }\end{array}$

\begin{abstract}
Short Title
Making and Curing Concrete Test Specimens in the Field

Sampling Fresh Concrete

Slump of Portland Cement Concrete

Unit Weight, Yield, and Air Content (Gravimetric) of Concrete

Air Content of Freshly Mixed Concrete by the Pressure Method

Compressive Strength of Cylindrical Concrete Specimens
\end{abstract}

NVLAP LAB CODE 0190

\title{
CORONET CARPETS \\ CORONET INDUSTRIES
}

P.O. Box 1248, Cleveland Drive, Dalton, GA 30720

Winfred L. Jones $\quad$ Phone: 404-259-4511

Accreditation Renewa! Date: January 1, 1986

$\begin{array}{lll}\text { NVLAP Code } & \text { Designation } & \text { Short Title } \\ \text { 03/C01 } & \text { AATCC 16E } & \text { Colorfastness to Light (Xenon Arc) } \\ \text { 03/C02 } & \text { AATCC 8 } & \text { Colorfastness to Crocking } \\ \text { 03/D01 } & \text { ASTM D418 } & \begin{array}{l}\text { Pile Yarn Floor Covering Construction } \\ \text { Pile Weight - Uncoated (Section 8) } \\ \end{array} \\ & & \begin{array}{l}\text { Pile Weight - Coated (Section 9) } \\ \text { Pile Thickness - (Sections 10 \& 11) }\end{array} \\ & & \text { Tuft Height - (Section 13) } \\ & & \text { Shrinkage } \\ \text { 03/D02 } & \text { DDD-C-95A } & \text { Tuft Bind of Floor Coverings } \\ \text { 03/S01 } & \text { ASTM D1335 } & \\ & \text { Federal Test Method } & \text { Textile Test Method - Breaking Strength } \\ & \text { Standard 191-5100 } & \text { Textile Test Method - Delamination } \\ & \text { 191-5950 } & \text { Methenamine Pill Test }\end{array}$

NVLAP LAB CODE 0191

STS CONSULTANTS, LTD.

111 Pfingsten Road, Northbrook, IL 60062

Michael T. Russell Phone: 312-272-6520
Accreditation Renewal Date: January 1, 1986

$\begin{array}{ll}\text { NVLAP Code } & \text { Designation } \\ \text { 02/M01 } & \text { ASTM C31 } \\ \text { 02/M03 } & \text { ASTM C172 } \\ \text { 02/P01 } & \text { ASTM C143 } \\ .02 / \mathrm{W} 01 & \text { ASTM C138 } \\ 02 / \mathrm{A} 01 & \text { ASTM C231 }\end{array}$

Short Title

Making and Curing Concrete Test Specimens in the Field

Sampling Fresh Concrete

Slump of Portland Cement Concrete

Unit Weight, Yield, and Air Content (Gravimetric) of Concrete

Air Content of Freshly Mixed Concrete by the Pressure Method 


$\begin{array}{ll}\text { NVLAP Code } & \text { Designation } \\ \text { 02/S01 } & \text { ASTM C39 } \\ 02 / \mathrm{A} 02 & \text { ASTM C173 }\end{array}$

\section{Short Title}

Compressive Strength of Cylindrical Concrete Specimens

Air Content of Freshly Mixed Concrete by the Volumetric Method

NVLAP LAB CODE 0192

\section{SMITH-EMERY COMPANY}

781 East Washington Boulevard, Los Angeles, CA 90021

George E. Battey, Jr. Phone: 213-749-3411

Accreditation Renewal Date: January 1, 1986

$\begin{array}{ll}\text { NVLAP Code } & \text { Designation } \\ \text { 02/M01 } & \text { ASTM C31 } \\ \text { 02/M03 } & \text { ASTM C172 } \\ \text { 02/P01 } & \text { ASTM C143 } \\ \text { 02/W01 } & \text { ASTM C138 } \\ \text { 02/A01 } & \text { ASTM C231 } \\ \text { 02/S01 } & \text { ASTM C39 } \\ \text { 02/A02 } & \text { ASTM C173 }\end{array}$

Short Title

Making and Curing Concrete Test Specimens in the Field

Sampling Fresh Concrete

Slump of Portland Cement Concrete

Unit Weight, Yield, and Air Content (Gravimetric) of Concrete

Air Content of Freshly Mixed Concrete by the Pressure Method

Compressive Strength of Cylindrical Concrete Specimens

Air Content of Freshly Mixed Concrete by the Volumetric Method

NVLAP LAB CODE 0193

SHAW INDUSTRIES, INC.

Plant \#4, S. Hamilton St. Ext., P.O. Drawer 2128, Dalton, GA 30720

Carey Mitchell Phone: 404-278-3812

Accreditation Renewal Date: January 1, 1986

$\begin{array}{ll}\text { NVLAP Code } & \text { Designation } \\ 03 / \mathrm{C} 01 & \text { AATCC 16E } \\ 03 / \mathrm{C} 02 & \text { AATCC } 8 \\ 03 / \mathrm{D} 01 & \text { ASTM D418 }\end{array}$

03/D02

03/S01

03/F03
DDD-C-95A

ASTM D1335

Federal Test Method

Standard 191-5100

191-5950

DoC FF $1-70$
Short Title

Colorfastness to Light (Xenon Arc)

Colorfastness to Crocking

Pile Yarn Floor Covering Construction

Pile Weight - Uncoated (Section 8)

Pile Weight - Coated (Section 9)

Pile Thickness - (Sections 10 \& 11)

Tuft Height - (Section 13)

Shrinkage

Tuft Bind of Floor Coverings

Textile Test Method - Breaking Strength

Textile Test Method - Delamination

Methenamine Pill Test

NVLAP LAB CODE 0195

\section{GARCO TESTING LABORATORIES \\ 532 West 3560 South, Salt Lake City, UT 84107 \\ Douglas L. Watson Phone: 801-266-4498}

Accreditation Renewal Date: January 1, 1986

$\begin{array}{ll}\text { NVLAP Code } & \text { Designation } \\ \text { 02/M01 } & \text { ASTM C31 } \\ \text { 02/M03 } & \text { ASTM C172 } \\ \text { 02/P01 } & \text { ASTM C143 } \\ \text { 02/W01 } & \text { ASTM C138 } \\ \text { 02/A01 } & \text { ASTM C231 } \\ \text { 02/S01 } & \text { ASTM C39 } \\ \text { 02/A02 } & \text { ASTM C173 }\end{array}$

Short Title

Making and Curing Concrete Test Specimens in the Field

Sampling Fresh Concrete

Slump of Portland Cement Concrete

Unit Weight, Yield, and Air Content (Gravimetric) of Concrete

Air Content of Freshly Mixed Concrete by the Pressure Method

Compressive Strength of Cylindrical Concrete Specimens

Air Content of Freshly Mixed Concrete by the Volumetric Method 


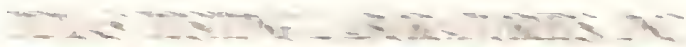

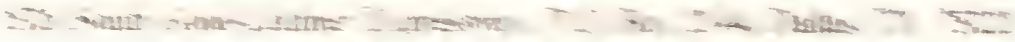

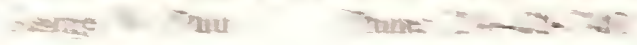
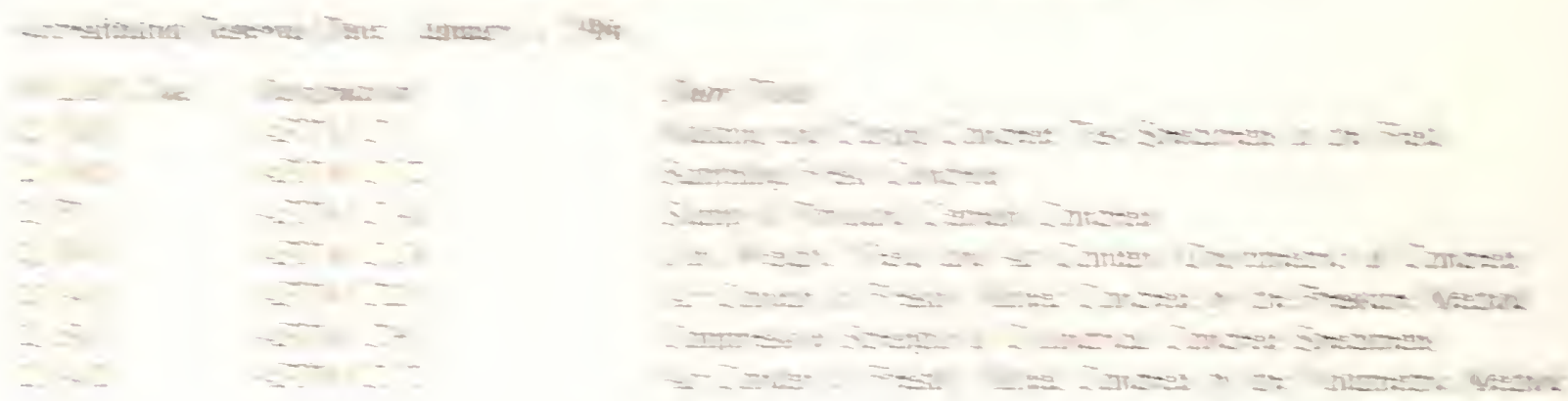

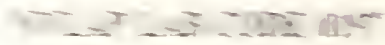

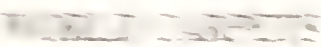

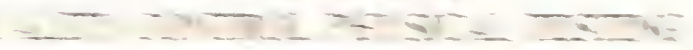

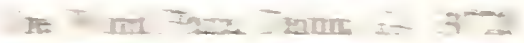

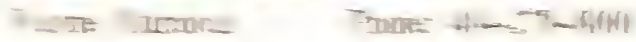

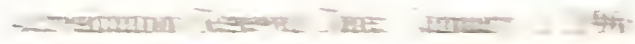

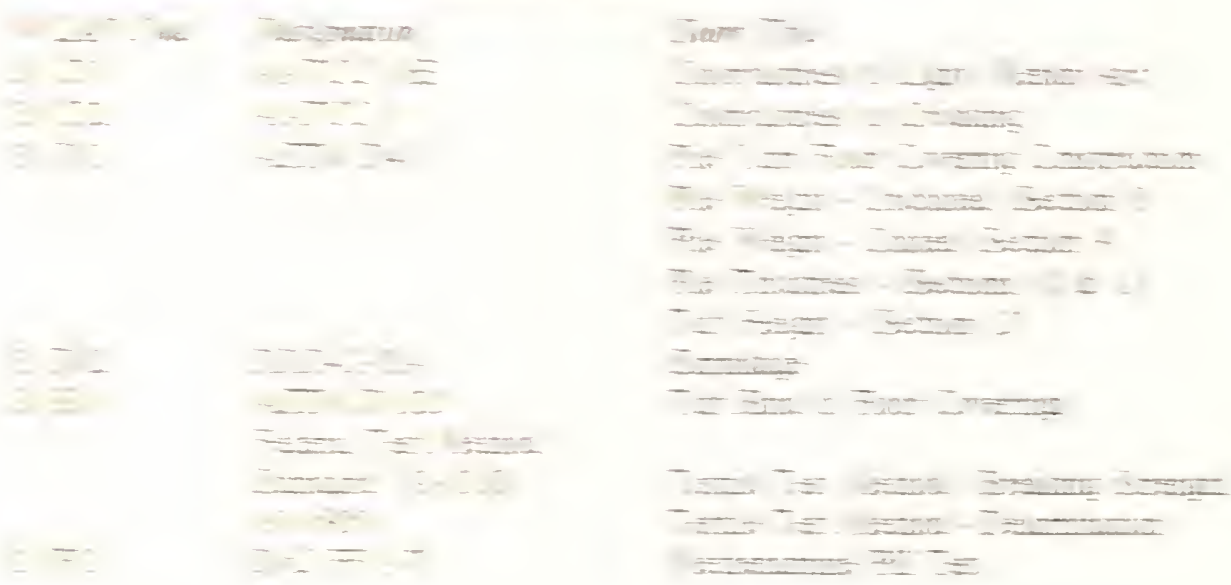

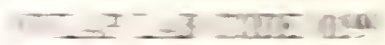

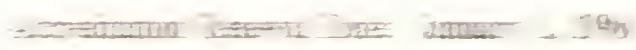

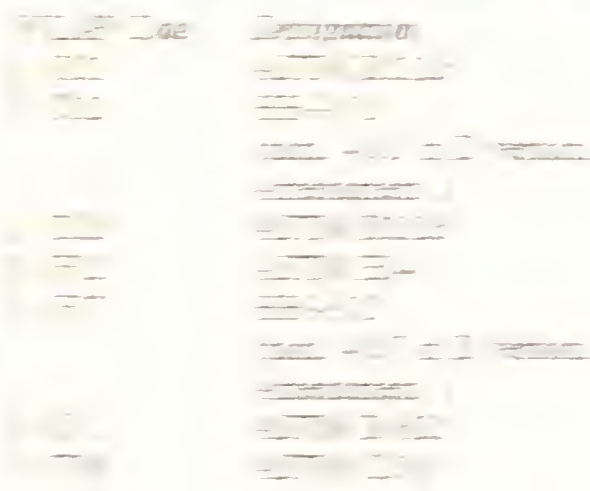

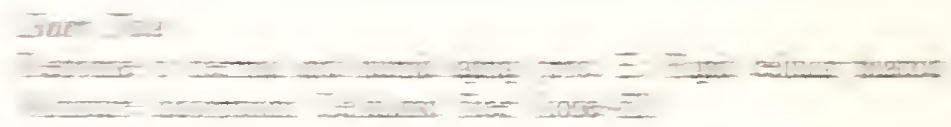




\title{
PITTSBURGH TESTING LABORATORY
}

850 Poplar Street, Pittsburgh, PA 15220

William H. Levelius Phone: 412-922-4000

Accreditation Renewal Date: October 1, 1985

$\begin{array}{ll}\text { NVLAP Code } & \text { Designation } \\ \text { 02/M01 } & \text { ASTM C31 } \\ \text { 02/M03 } & \text { ASTM C172 } \\ \text { 02/P01 } & \text { ASTM C143 } \\ \text { 02/W01 } & \text { ASTM C138 } \\ \text { 02/A01 } & \text { ASTM C231 } \\ \text { 02/S01 } & \text { ASTM C39 } \\ \text { 02/A02 } & \text { ASTM C173 }\end{array}$

\section{Short Title}

Making and Curing Concrete Test Specimens in the Field

Sampling Fresh Concrete

Slump of Portland Cement Concrete

Unit Weight, Yield, and Air Content (Gravimetric) of Concrete Air Content of Freshly Mixed Concrete by the Pressure Method Compressive Strength of Cylindrical Concrete Specimens

Air Content of Freshly Mixed Concrete by the Volumetric Method

NVLAP LAB CODE 0203

\section{CAL MAT CO.}

\section{CONROCK DIVISION TESTING LABORATORY}

P.O. Box 2950, Terminal Annex, Los Angeles, CA 90051

James Neal Van Nest

Phone: 213-258-2777

Accreditation Renewal Date: January 1, 1986

$\begin{array}{ll}\text { NVLAP Code } & \text { Designation } \\ \text { 02/M01 } & \text { ASTM C31 } \\ 02 / \mathrm{MO} 3 & \text { ASTM C172 } \\ 02 / \mathrm{P} 01 & \text { ASTM C143 } \\ 02 / \mathrm{W} 01 & \text { ASTM C138 } \\ 02 / \mathrm{A} 01 & \text { ASTM C231 } \\ 02 / \mathrm{S} 01 & \text { ASTM C39 } \\ 02 / \mathrm{A02} & \text { ASTM C173 }\end{array}$

\author{
Short Title \\ Making and Curing Concrete Test Specimens in the Field \\ Sampling Fresh Concrete \\ Slump of Portland Cement Concrete \\ Unit Weight, Yield, and Air Content (Gravimetric) of Concrete \\ Air Content of Freshly Mixed Concrete by the Pressure Method \\ Compressive Strength of Cylindrical Concrete Specimens \\ Air Content of Freshly Mixed Concrete by the Volumetric Method
}

NVLAP LAB CODE 0205

\author{
WEST VIRGINIA DEPT OF HIGHWAYS \\ MATERIALS CONTROL, SOIL \& TESTING \\ 312 Michigan Avenue, Charleston, WV 25311 \\ Thomas M. Dugan \\ Phone: 304-348-3160
}

Accreditation Renewal Date: April 1, 1985

$\begin{array}{ll}\text { NVLAP Code } & \text { Designation } \\ \text { 02/M01 } & \text { ASTM C31 } \\ \text { 02/M03 } & \text { ASTM C172 } \\ \text { 02/P01 } & \text { ASTM C143 } \\ \text { 02/W01 } & \text { ASTM C138 } \\ \text { 02/A01 } & \text { ASTM C231 } \\ \text { 02/S01 } & \text { ASTM C39 } \\ \text { 02/A02 } & \text { ASTM C173 }\end{array}$

Short Title

Making and Curing Concrete Test Specimens in the Field

Sampling Fresh Concrete

Slump of Portland Cement Concrete

Unit Weight, Yield, and Air Content (Gravimetric) of Concrete

Air Content of Freshly Mixed Concrete by the Pressure Method

Compressive Strength of Cylindrical Concrete Specimens

Air Content of Freshly Mixed Concrete by the Volumetric Method 


\title{
R. W. SIDLEY, INC. QUALITY CONTROL LABORATORY \\ 6900 Madison Road, Thompson, $\mathrm{OH} 44086$ \\ Lawrence McCune Phone: 216-298-3232
}

Accreditation Renewal Date: January 1, 1986

$\begin{array}{ll}\text { NVLAP Code } & \text { Designation } \\ 02 / \mathrm{M} 01 & \text { ASTM C31 } \\ 02 / \mathrm{M} 03 & \text { ASTM C172 } \\ 02 / \mathrm{P} 01 & \text { ASTM C143 } \\ 02 / \mathrm{W} 01 & \text { ASTM C138 } \\ 02 / \mathrm{A} 01 & \text { ASTM C231 } \\ 02 / \mathrm{S} 01 & \text { ASTM C39 } \\ 02 / \mathrm{A} 02 & \text { ASTM C173 }\end{array}$

\begin{abstract}
Short Title
Making and Curing Concrete Test Specimens in the Field

Sampling Fresh Concrete

Slump of Portland Cement Concrete

Unit Weight, Yield, and Air Content (Gravimetric) of Concrete

Air Content of Freshly Mixed Concrete by the Pressure Method

Compressive Strength of Cylindrical Concrete Specimens

Air Content of Freshly Mixed Concrete by the Volumetric Method
\end{abstract}

NVLAP LAB CODE 0208

\section{GULF COAST TESTING LABORATORY, INC. \\ 1205 North Tancahua Street, Corpus Christi, TX 78401 \\ Doyne Reynolds Phone: 512-882-5411}

Accreditation Renewal Date: January 1, 1986

$\begin{array}{ll}\text { NVLAP Code } & \text { Designation } \\ \text { 02/M01 } & \text { ASTM C31 } \\ \text { 02/M03 } & \text { ASTM C172 } \\ \text { 02/P01 } & \text { ASTM C143 } \\ \text { 02/W01 } & \text { ASTM C138 } \\ \text { 02/A01 } & \text { ASTM C231 } \\ \text { 02/S01 } & \text { ASTM C39 } \\ \text { 02/A02 } & \text { ASTM C173 }\end{array}$

Short Title

Making and Curing Concrete Test Specimens in the Field

Sampling Fresh Concrete

Slump of Portland Cement Concrete

Unit Weight, Yield, and Air Content (Gravimetric) of Concrete

Air Content of Freshly Mixed Concrete by the Pressure Method

Compressive Strength of Cylindrical Concrete Specimens

Air Content of Freshly Mixed Concrete by the Volumetric Method

NVLAP LAB CODE 0210

\section{INSTA-FOAM PRODUCTS, INC. \\ 1500 Cedarwood Drive, Joliet, IL 60435 \\ Kenneth A. Pugh Phone: 815-741-6851}

Accreditation Renewal Date: January 1, 1986

$\begin{array}{ll}\text { NVLAP Code } & \text { Designation } \\ 01 / \text { D15 } & \text { ASTM D756 } \\ 01 / \text { D16 } & \text { ASTM D756 } \\ 01 / \text { D17 } & \text { ASTM D756 } \\ 01 / \text { D18 } & \text { ASTM D1622 } \\ 01 / \text { D20 } & \text { ASTM D2126 } \\ 01 / \text { D22 } & \text { ASTM D2126 } \\ 01 / \text { D23 } & \text { ASTM D2842 } \\ 01 / \text { D27 } & \text { ASTM D2126 } \\ 01 / \text { D28 } & \text { ASTM D2126 } \\ 01 / \text { S11 } & \text { ASTM D1621 } \\ 01 / \text { T05 } & \text { ASTM C335 } \\ 01 / \text { T06 } & \text { ASTM C518 } \\ 01 / \text { V04 } & \text { ASTM E96 }\end{array}$

Short Title

Weight and shape changes; Accelerated service (proc. A); Plastics

Weight and shape changes; Accelerated service (proc. B); Plastics

Weight and shape changes; Accelerated service (proc. E); Plastics

Apparent density; Rigid cellular plastics

Response to thermal and humid aging (proc. D); Rigid cellular plastics

Response to thermal and humid aging (proc. F); Rigid cellular plastics

Water absorption; Rigid cellular plastics

Response to thermal and humid aging (proc. C); Rigid cellular plastics

Response to thermal and humid aging (proc. G); Rigid cellular plastics

Compressive properties; Rigid cellular plastics (proc. A-Crosshead)

Thermal conductivity; Pipe insulation

Thermal transmission properties; Heat flow meter

Water vapor transmission; Thin sheets (proc. A) 


\title{
CONSTRUCTION MATERIALS CONSULTANTS, INC. \\ 1000 West Fillmore Street, Colorado Springs, CO 80907 \\ Ivan A. Vanaken Phone: 303-632-2588
}

Accreditation Renewal Date: July 1, 1985

$\begin{array}{ll}\text { NVLAP Code } & \text { Designation } \\ \text { 02/M01 } & \text { ASTM C31 } \\ \text { 02/M03 } & \text { ASTM C172 } \\ \text { 02/P01 } & \text { ASTM C143 } \\ \text { 02/W01 } & \text { ASTM C138 } \\ \text { 02/A01 } & \text { ASTM C231 } \\ \text { 02/S01 } & \text { ASTM C39 } \\ \text { 02/A02 } & \text { ASTM C173 }\end{array}$

Short Title

Making and Curing Concrete Test Specimens in the Field

Sampling Fresh Concrete

Slump of Portland Cement Concrete

Unit Weight, Yield, and Air Content (Gravimetric) of Concrete

Air Content of Freshly Mixed Concrete by the Pressure Method

Compressive Strength of Cylindrical Concrete Specimens

Air Content of Freshly Mixed Concrete by the Volumetric Method

NVLAP LAB CODE 0216

\section{UNITED STATES GYPSUM COMPANY, RESEARCH CENTER}

700 North Highway 45, Libertyville, IL 60048

William F. Porter

Phone: 312-362-9797

Accreditation Renewal Date: July 1, 1985

NVLAP Code Designation

01/T06 ASTM C518
Short Title

Thermal transmission properties; Heat flow meter

NVLAP LAB CODE 0218

\section{APACHE BUILDING PRODUCTS COMPANY \\ 2025 East Linden Avenue, Linden, NJ 07036 \\ Dennis W. Rosato \\ Phone: 201-486-6723}

Accreditation Renewal Date: October 1, 1985

$\begin{array}{ll}\text { NVLAP Code } & \text { Designation } \\ 01 / \mathrm{D} 18 & \text { ASTM D1622 } \\ 01 / \mathrm{D} 21 & \text { ASTM D2126 } \\ 01 / \mathrm{D} 27 & \text { ASTM D2126 } \\ 01 / \text { S11 } & \text { ASTM D1621 } \\ 01 / \text { T06 } & \text { ASTM C518 }\end{array}$

\begin{abstract}
Short Title
Apparent density; Rigid cellular plastics

Response to thermal and humid aging (proc. E); Rigid cellular plastics

Response to thermal and humid aging (proc. C); Rigid cellular plastics

Compressive properties; Rigid cellular plastics (proc. A-Crosshead)

Thermal transmission properties; Heat flow meter
\end{abstract}

NVLAP LAB CODE 0220

\section{STRATTON LABORATORIES}

Highway 61, South, P.O. Box 1007, Cartersville, GA 30120

Jack R. Kilgore Phone: 404-382-9350

Accreditation Renewal Date: October 1, 1985

$\begin{array}{ll}\text { NVIAP Code } & \text { Designation } \\ \text { 03/S01 } & \text { ASTM D1335 } \\ & \text { Federal Test Method } \\ & \text { Standard 191-5100 } \\ & \text { 191-5950 } \\ \text { 03/F03 } & \text { DoC FF 1-70 } \\ \text { 03/F04 } & \text { ASTM E648 }\end{array}$

Short Title

Tuft Bind of Floor Coverings

Textile Test Method - Breaking Strength

Textile Test Method - Delamination

Methenamine Pill Test

Radiant Panel (Carpet) 


\section{SALEM CARPET LABORATORY}

P.O. Box 10, Chatsworth, GA 30736

Michael A. Corbin Phone: 404-935-2241

Accreditation Renewal Date: Jully 1, 1985

$\begin{array}{ll}\text { NVLAP Code } & \begin{array}{l}\text { Designation } \\ \text { 03/C01 }\end{array} \\ \text { 03/C02 } & \text { AATCC 16E } \\ \text { 03/D01 } & \text { ASTM D418 } \\ & \\ & \\ & \\ \text { 03/D02 } & \text { DDD-C-95A } \\ \text { 03/S01 } & \text { ASTM D1335 } \\ & \text { Federal Test Method } \\ & \text { Standard 191-5100 } \\ & \text { 191-5950 } \\ \text { 03/F03 } & \text { DoC FF 1-70 } \\ \text { 03/F04 } & \text { ASTM E648 }\end{array}$

\author{
Short Title \\ Colorfastness to Light (Xenon Arc) \\ Colorfastness to Crocking \\ Pile Yarn Floor Covering Construction \\ Pile Weight - Uncoated (Section 8) \\ Pile Weight - Coated (Section 9) \\ Pile Thickness - (Sections 10 \& 11) \\ Tuft Height - (Section 13) \\ Shrinkage \\ Tuft Bind of Floor Coverings \\ Textile Test Method - Breaking Strength \\ Textile Test Method - Delamination \\ Methenamine Pill Test \\ Radiant Panel (Carpet)
}

NVLAP LAB CODE 0223

\section{PFS CORPORATION \\ 2402 Daniels Street, Madison, WI 53704 \\ Ed Starostovic \\ Phone: 608-221-3361}

\begin{tabular}{|c|c|c|c|}
\hline \multirow[b]{2}{*}{ NVLAP Code } & \multirow[b]{2}{*}{$\begin{array}{l}\text { Short Title } \\
\text { PHYSICAL/FIRE TEST GROUP (04/F00) }\end{array}$} & \multicolumn{2}{|c|}{ Accreditation Renewal Date: January 1, 198} \\
\hline & & $\begin{array}{l}\text { Section of UL } 737 \\
\text { 5th Edition } \\
\text { (March 1, 1982) }\end{array}$ & $\begin{array}{l}\text { Section of UL } 1482 \\
\text { 2nd Edition } \\
\text { (January 24, 1983) }\end{array}$ \\
\hline 04/F01 & Test Installation & 8 & 8 \\
\hline 04/F02 & Temperature Measurement & 9 & 9 \\
\hline 04/F04 & Radiant Fire Test & 11 & 11 \\
\hline 04/F05 & Coal Fire Test & & 14 \\
\hline 04/F06 & Brand Fire Test & 12 & 12 \\
\hline 04/F07 & Flash Fire Test & 13 & 13 \\
\hline 04/F08 & Strength Tests & 15 & 16 \\
\hline 04/F09 & Stability Test & 16 & 16 \\
\hline 04/F10 & Glazing Test & 14 & 15 \\
\hline $\begin{array}{l}\text { 04/M01 } \\
\text { 04/M02 } \\
\text { 04/M03 }\end{array}$ & $\begin{array}{l}\text { MOBILE HOME TEST GROUP }(04 / \mathrm{M} 00) \\
\text { Test Installation } \\
\text { Toxic Gas } \\
\text { Drop Test }\end{array}$ & $\begin{array}{l}17 \\
17 \\
17\end{array}$ & $\begin{array}{l}17 \\
17 \\
17\end{array}$ \\
\hline & ELECTRICAL TEST GROUP (04/E00) & & \\
\hline 04/E01 & Test Voltages & 33 & 33 \\
\hline 04/E02 & Temperature Measurements, Electrical Components & 34 & 34 \\
\hline 04/E03 & Input Test & 35 & 35 \\
\hline 04/E04 & Temperature Test, Electrical Components & 36 & 36 \\
\hline 04/E05 & Leakage Current & 38 & 38 \\
\hline 04/E06 & Dielectric Withstand & 37 & 37 \\
\hline 04/E07 & Locked Rotor (Stalled Motor) Temperature & 39 & 39 \\
\hline 04/E08 & Power Cord Strain Relief & 40 & 40 \\
\hline
\end{tabular}




\section{ARNOLD GREENE TESTING LABORATORIES \\ A DIVISION OF CONAM INSPECTION \\ 2 Millbury Street, Auburn, MA 01501A \\ Robert J. Halliday \\ Phone: 617-235-7330}

Accreditation Renewal Date: January 1, 1986

\begin{tabular}{|c|c|c|c|}
\hline NVLAP Code & $\begin{array}{l}\text { Short Title } \\
\text { PHYSICAL/FIRE TEST GROUP (04/F00) }\end{array}$ & (March 1, 1982) & (January 24, 1983) \\
\hline 04/F01 & Test Installation & 8 & 8 \\
\hline 04/F02 & Temperature Measurement & 9 & 9 \\
\hline 04/F04 & Radiant Fire Test & 11 & 11 \\
\hline 04/F05 & Coal Fire Test & & 14 \\
\hline 04/F06 & Brand Fire Test & 12 & 12 \\
\hline 04/F07 & Flash Fire Test & 13 & 13 \\
\hline 04/F08 & Strength Tests & 15 & 16 \\
\hline 04/F09 & Stability Test & 16 & 16 \\
\hline $04 / F 10$ & Glazing Test & 14 & 15 \\
\hline $\begin{array}{l}\text { 04/M01 } \\
\text { 04/M02 } \\
\text { 04/M03 }\end{array}$ & $\begin{array}{l}\text { MOBILE HOME TEST GROUP }(04 / \text { M00) } \\
\text { Test Installation } \\
\text { Toxic Gas } \\
\text { Drop Test }\end{array}$ & $\begin{array}{l}17 \\
17 \\
17\end{array}$ & $\begin{array}{l}17 \\
17 \\
17\end{array}$ \\
\hline & ELECTRICAL TEST GROUP (04/E00) & & \\
\hline 04/E01 & Test Voltages & 33 & 33 \\
\hline 04/E02 & Temperature Measurements, Electrical Components & 34 & 34 \\
\hline 04/E03 & Input Test & 35 & 35 \\
\hline 04/E04 & Temperature Test, Electrical Components & 36 & 36 \\
\hline 04/E05 & Leakage Current & 38 & 38 \\
\hline 04/E06 & Dielectric Withstand & 37 & 37 \\
\hline 04/E07 & Locked Rotor (Stalled Motor) Temperature & 39 & 39 \\
\hline 04/E08 & Power Cord Strain Relief & 40 & 40 \\
\hline
\end{tabular}

NVLAP LAB CODE 0226

WISS, JANNEY, ELSTNER AND ASSOCIATES, INC. 330 Pfingsten Road, Northbrook, IL 60062

Jerry G. Stockbridge Phone: 312-272-7400

Accreditation Renewal Date: July 1, 1984

NVLAP Code Designation

01/T04 ASTM C236
Short Title

Thermal conductance; Guarded hot box

\section{RIVERBANK ACOUSTICAL LABORATORIES \\ P.O.Box 189, 1512 Batavia Avenue, Geneva, IL 60134 John W. Kopec \\ Phone: 312-232-0104}

Accreditation Renewal Date: April 1, 1985

$\begin{array}{ll}\text { NVLAP Code } & \text { Designation } \\ \text { 08/P03 } & \text { ASTM C423-84a } \\ \text { 08/P05 } & \text { ASTM C523-68 (81) } \\ \text { 08/P06 } & \text { ASTM E90-83 }\end{array}$

\section{Short Title}

Sound Absorption and Sound Absorption Coefficients

Light Reflectance of Acoustical Materials

Airborne Sound Transmission Loss of Building Partitions 


$\begin{array}{ll}\text { NVLAP Code } & \text { Designation } \\ 08 / \text { P07 } & \text { ASTM E492-82 } \\ 08 / \text { P10 } & \text { ANSI S1.31-80 } \\ 08 / \text { P17 } & \text { ISO 3741-75 } \\ & \\ 08 / \text { E01 } & \begin{array}{l}\text { ANSI B71.1-80 } \\ \text { (para. } 9 \text { and 21) }\end{array}\end{array}$

Short Title

Impact Sound Transmission Through Floor-Ceiling Assemblies

Sound Power Levels, Broad-Band Noise Sources in Reverberation Rooms (100-10,000 Hz)

Sound Power Levels, Broad-Band Sources in Reverberation Rooms $(100-10,000 \mathrm{~Hz})$

Sound Level Tests; Power Lawn

Mowers, Lawn and Garden Tractors and Lawn Tractors

NVLAP LAB CODE 0228

\section{ARMSTRONG WORLD INDUSTRIES \\ TECHNICAL CENTER, ACOUSTICS LABORATORY \\ 2500 Columbia Avenue, P.O.Box 3511, Lancaster, PA 17604 \\ G. Robert Spalding \\ Phone: 717-397-0611}

Accreditation Renewal Date: January 1, 1986
NVLAP Code
Designation
ASTM C423-84a
ANSI/ASTM E492-82
Short Title
Sound Absorption and Sound Absorption Coefficients
Impact Sound Transmission Through Floor-Ceiling Assemblies
08/P07

NVLAP LAB CODE 0229

\section{GOLD BOND BUILDING PRODUCTS \\ A NATIONAL GYPSUM DIVISION, RESEARCH CENTER \\ 1650 Military Road, Buffalo, NY 14217 \\ Joseph Volk Phone: 716-873-9750}

Accreditation Renewal Date: April 1, 1985

$\begin{array}{ll}\text { NVLAP Code } & \text { Designation } \\ \text { 08/P03 } & \text { ASTM C423-84a } \\ 08 / \text { P05 } & \text { ASTM C523-68 (81) } \\ 08 / \text { P06 } & \text { ASTM E90-83 } \\ 08 / \text { E21 } & \text { AMA-1-II-67 }\end{array}$

Short Title

Sound Absorption and Sound Absorption Coefficients

Light Reflectance of Acoustical Materials

Airborne Sound Transmission Loss of Building Partitions

Ceiling Sound Transmission Test by Two-Room Method

NVLAP LAB CODE 0230

\section{VIRGINIA CONCRETE LABORATORY 6555 Edsall Road, Box 666, Springfield, VA 22150 \\ Richard A. Buckelew Phone: 703-354-6111}

Accreditation Renewal Date: April 1, 1984

$\begin{array}{ll}\text { NVLAP Code } & \text { Designation } \\ \text { 02/M01 } & \text { ASTM C31 } \\ \text { 02/M03 } & \text { ASTM C172 } \\ \text { 02/P01 } & \text { ASTM C143 } \\ \text { 02/W01 } & \text { ASTM C138 } \\ \text { 02/A01 } & \text { ASTM C231 } \\ \text { 02/S01 } & \text { ASTM C39 } \\ \text { 02/A02 } & \text { ASTM C173 }\end{array}$

Short Title

Making and Curing Concrete Test Specimens in the Field

Sampling Fresh Concrete

Slump of Portland Cement Concrete

Unit Weight, Yield, and Air Content (Gravimetric) of Concrete

Air Content of Freshly Mixed Concrete by the Pressure Method

Compressive Strength of Cylindrical Concrete Specimens

Air Content of Freshly Mixed Concrete by the Volumetric Method 


\section{RITCHIE LABORATORIES}

\section{North Mosley, P.O. Box 4048, Wichita, KS 67204}

Donald J. Brockel Phone: 316-263-9937

Accreditation Renewal Date: January 1, 1986

$\begin{array}{ll}\text { NVLAP Code } & \text { Designation } \\ \text { 02/M01 } & \text { ASTM C31 } \\ \text { 02/M03 } & \text { ASTM C172 } \\ \text { 02/P01 } & \text { ASTM C143 } \\ \text { 02/W01 } & \text { ASTM C138 } \\ \text { 02/A01 } & \text { ASTM C231 } \\ \text { 02/S01 } & \text { ASTM C39 } \\ \text { 02/A02 } & \text { ASTM C173 }\end{array}$

Short Title

Making and Curing Concrete Test Specimens in the Field

Sampling Fresh Concrete

Slump of Portland Cement Concrete

Unit Weight, Yield, and Air Content (Gravimetric) of Concrete

Air Content of Freshly Mixed Concrete by the Pressure Method

Compressive Strength of Cylindrical Concrete Specimens

Air Content of Freshly Mixed Concrete by the Volumetric Method

NVLAP LAB CODE 0233

\section{STS CONSULTANTS, LTD. FAIRFAX VA OFFICE}

2929-C Eskridge Road, Fairfax, VA 22031

Henry L. Lucas

Phone: 703-698-5300

Accreditation Renewal Date: October 1, 1985

$\begin{array}{ll}\text { NVLAP Code } & \text { Designation } \\ \text { 02/M01 } & \text { ASTM C31 } \\ \text { 02/M03 } & \text { ASTM C172 } \\ \text { 02/P01 } & \text { ASTM C143 } \\ \text { 02/W01 } & \text { ASTM C138 } \\ \text { 02/A01 } & \text { ASTM C231 } \\ \text { 02/S01 } & \text { ASTM C39 }\end{array}$

\begin{abstract}
Short Title
Making and Curing Concrete Test Specimens in the Field

Sampling Fresh Concrete

Slump of Portland Cement Concrete

Unit Weight, Yield, and Air Content (Gravimetric) of Concrete

Air Content of Freshly Mixed Concrete by the Pressure Method

Compressive Strength of Cylindrical Concrete Specimens
\end{abstract}

NVLAP LAB CODE 0235

\section{PACIFIC INSPECTION AND RESEARCH LABORATORY, INC. 4076 148th Avenue North East, Redmond, WA 98052 \\ Ronald J. Weisel Phone: 206-881-7668}

Accreditation Renewal Date: October 1, 1985

$\begin{array}{ll}\text { NVLAP Code } & \text { Short Title } \\ & \text { PHYSICAL/FIRE TEST GROUP (04/F00) } \\ \text { 04/F01 } & \text { Test Installation } \\ \text { 04/F02 } & \text { Temperature Measurement } \\ \text { 04/F04 } & \text { Radiant Fire Test } \\ \text { 04/F05 } & \text { Coal Fire Test } \\ \text { 04/F06 } & \text { Brand Fire Test } \\ \text { 04/F07 } & \text { Flash Fire Test } \\ \text { 04/F08 } & \text { Strength Tests } \\ \text { 04/F09 } & \text { Stability Test } \\ \text { 04/F10 } & \text { Glazing Test } \\ & \text { MOBILE HOME TEST GROUP (04/M00) } \\ \text { 04/M01 } & \text { Test Installation } \\ \text { 04/M02 } & \text { Toxic Gas } \\ \text { 04/M03 } & \text { Drop Test }\end{array}$

$\begin{array}{cc}\text { Section of } U L 737 & \text { Section of UL 1482 } \\ \text { 5th Edition } & \text { 2nd Edition } \\ \text { (March 1, 1982) } & \text { (January 24, 1983) }\end{array}$

$\begin{array}{rr}8 & 8 \\ 9 & 9 \\ 11 & 11 \\ & 14 \\ 12 & 12 \\ 13 & 13 \\ 15 & 16 \\ 16 & 16 \\ 14 & 15\end{array}$

$\begin{array}{ll}17 & 17 \\ 17 & 17 \\ 17 & 17\end{array}$


NVLAP Code Short Title

ELECTRICAL TEST GROUP (04/E00)

04/E01

04/E02

$04 / \mathrm{E} 03$

04/E04

04/E05

04/E06

$04 / \mathrm{E} 07$

04/E08
Test Voltages

Temperature Measurements, Electrical Components

Input Test

Temperature Test, Electrical Components

Leakage Current

Dielectric Withstand

Locked Rotor (Stalled Motor) Temperature

Power Cord Strain Relief 5th Edition

(March 1, 1982)

33
34
35
36
38
37
39
40

NVLAP LAB CODE 0237 2nd Edition

(January 24, 1983)

$\begin{array}{ll}33 & 33 \\ 34 & 34 \\ 35 & 35 \\ 36 & 36 \\ 38 & 38 \\ 37 & 37 \\ 39 & 39 \\ 40 & 40\end{array}$

\title{
PITTSBURGH TESTING LABORATORY SYRACUSE NY PLANT LABORATORY 6159 East Mallory Road, Syracuse, NY 13057 \\ W.J. Peters Phone: 315-437-7043
}

Accreditation Renewal Date: April 1, 1985

$\begin{array}{ll}\text { NVLAP Code } & \text { Designation } \\ \text { 02/M01 } & \text { ASTM C31 } \\ \text { 02/M03 } & \text { ASTM C172 } \\ 02 / \mathrm{P} 01 & \text { ASTM C143 } \\ \text { 02/W01 } & \text { ASTM C138 } \\ 02 / \mathrm{A} 01 & \text { ASTM C231 } \\ 02 / \mathrm{S} 01 & \text { ASTM C39 } \\ 02 / \mathrm{A02} & \text { ASTM C173 }\end{array}$

Short Title

Making and Curing Concrete Test Specimens in the Field

Sampling Fresh Concrete

Slump of Portland Cement Concrete

Unit Weight, Yield, and Air Content (Gravimetric) of Concrete

Air Content of Freshly Mixed Concrete by the Pressure Method

Compressive Strength of Cylindrical Concrete Specimens

Air Content of Freshly Mixed Concrete by the Volumetric Method

NVLAP LAB CODE 0239

\section{HUFCOR ACOUSTICAL LABORATORY HOUGH MANUFACTURING CORP.}

P.O. Box 591, 1205 Norwood Road, Janesville, WI 53547

Stanley Kowalczyk Phome: 608-756-1241

Accreditation Renewal Date: October 1, 1985
NVLAP Code Designation
08/P06 ASTM E90-83
Short Title
Airborne Sound Transmission Loss of Building Partitions

NVLAP LAB CODE 0240

\begin{abstract}
OMNI ENVIRONMENTAL SERVICES, INC. SOLID FUELS TESTING LAB

$10950 \mathrm{SW}$ 5th Street, Suite 245, Beaverton, OR 97005

Raymond W. Downey Phone: 503-643-3755
\end{abstract}

Accreditation Renewal Date: January 1, 1986

$\begin{array}{ll}\text { NVLAP Code } & \text { Short Title } \\ & \text { PHYSICAL/FIRE TEST GROUP (04/F00) } \\ \text { 04/F01 } & \text { Test Installation } \\ \text { 04/F02 } & \text { Temperature Measurement } \\ \text { 04/F04 } & \text { Radiant Fire Test }\end{array}$

Section of UL 737 Section of UL 1482

5th Edition

(March 1, 1982)

2nd Edition

(January 24, 1983) 
NVLAP Code Short Title

04/F05

04/F06

04/F07

04/F08

04/F09

04/F10

04/M01

04/M02

04/M03

04/E01

04/E02

04/E03

04/E04

04/E05

04/E06

04/E07

04/E08

Toxic Gas.

Drop Test

Input Test
Coal Fire Test

Brand Fire Test

Flash Fire Test

Strength Tests

Stability Test

Glazing Test

MOBILE HOME TEST GROUP (04/M00)

Test Installation

ELECTRICAL TEST GROUP (04/E00)

Test Voltages

Temperature Measurements, Electrical Components

Temperature Test, Electrical Components

Leakage Current

Dielectric Withstand

Locked Rotor (Stalled Motor) Temperature

Power Cord Strain Relief
Section of $U L 737$

5th Edition

(March 1, 1982)

Section of UL 1482

2nd Edition

(January 24, 1983)

$\begin{array}{ll} & 14 \\ 12 & 12 \\ 13 & 13 \\ 15 & 16 \\ 16 & 16 \\ 14 & 15\end{array}$

\section{7}

17

17

17

17

17

$\begin{array}{ll}33 & 33 \\ 34 & 34 \\ 35 & 35 \\ 36 & 36 \\ 38 & 38 \\ 37 & 37 \\ 39 & 39 \\ 40 & 40\end{array}$

NVLAP LAB CODE 0241

\section{WESTERN STATES TESTING DIVISION OF U.S. TESTING COMPANY, INC. \\ 3536 Oakdale Road, Modesto, CA 95355 \\ Harold Stevens \\ Phone: 209-527-2271}

Accreditation Renewal Date: October 1, 1985

$\begin{array}{ll}\text { NVLAP Code } & \text { Designation } \\ \text { 02/M01 } & \text { ASTM C31 } \\ \text { 02/M03 } & \text { ASTM C172 } \\ \text { 02/P01 } & \text { ASTM C143 } \\ \text { 02/W01 } & \text { ASTM C138 } \\ \text { 02/A01 } & \text { ASTM C231 } \\ \text { 02/S01 } & \text { ASTM C39 } \\ \text { 02/A02 } & \text { ASTM C173 }\end{array}$

Short Title

Making and Curing Concrete Test Specimens in the Field

Sampling Fresh Concrete

Slump of Portland Cement Concrete

Unit Weight, Yield, and Air Content (Gravimetric) of Concrete

Air Content of Freshly Mixed Concrete by the Pressure Method

Compressive Strength of Cylindrical Concrete Specimens

Air Content of Freshly Mixed Concrete by the Volumetric Method

NVLAP LAB CODE 0243

CUSTOM COATING, INC.

204 West Industrial Blvd., Dalton, GA 30720

Mike Calhoun

Phone: 404-277-3778

Accreditation Renewal Date: April 1, 1985

\section{NVLAP Code}

03/F03
Short Title

Methenamine Pill Test 


\section{NORTHWEST TESTING LABORATORIES, INC.}

P.O. Box 17126, Portland, OR 97217

Don Cave

Phone: 503-288-7086

Accreditation Renewal Date: January 1, 1986

\begin{tabular}{|c|c|c|c|}
\hline NVLAP Code & $\begin{array}{l}\text { Short Title } \\
\text { PHYSICAL/FIRE TEST GROUP (04/F00) }\end{array}$ & $\begin{array}{c}\text { 5th Edition } \\
\text { (March 1, 1982) }\end{array}$ & $\begin{array}{l}\text { 2nd Edition } \\
\text { (January 24, 1983) }\end{array}$ \\
\hline 04/F01 & Test Installation & 8 & 8 \\
\hline 04/F02 & Temperature Measurement & 9 & 9 \\
\hline 04/F04 & Radiant Fire Test & 11 & 11 \\
\hline 04/F05 & Coal Fire Test & & 14 \\
\hline 04/F06 & Brand Fire Test & 12 & 12 \\
\hline 04/F07 & Flash Fire Test & 13 & 13 \\
\hline 04/F08 & Strength Tests & 15 & 16 \\
\hline 04/F09 & Stability Test & 16 & 16 \\
\hline $04 /$ F10 & Glazing Test & 14 & 15 \\
\hline $\begin{array}{l}\text { 04/M01 } \\
\text { 04/M02 } \\
\text { 04/M03 }\end{array}$ & $\begin{array}{l}\text { MOBILE HOME TEST GROUP (04/M00) } \\
\text { Test Installation } \\
\text { Toxic Gas } \\
\text { Drop Test }\end{array}$ & $\begin{array}{l}17 \\
17 \\
17\end{array}$ & $\begin{array}{l}17 \\
17 \\
17\end{array}$ \\
\hline & ELECTRICAL TEST GROUP (04/E00) & & \\
\hline 04/E01 & Test Voltages & 33 & 33 \\
\hline 04/E02 & Temperature Measurements, Electrical Components & 34 & 34 \\
\hline $04 / \mathrm{E} 03$ & Input Test & 35 & 35 \\
\hline 04/E04 & Temperature Test, Electrical Components & 36 & 36 \\
\hline 04/E05 & Leakage Current & 38 & 38 \\
\hline 04/E06 & Dielectric Withstand & 37 & 37 \\
\hline 04/E07 & Locked Rotor (Stalled Motor) Temperature & 39 & 39 \\
\hline 04/E08 & Power Cord Strain Relief & 40 & 40 \\
\hline
\end{tabular}

NVLAP LAB CODE 0245

R. F. GEISSER \& ASSOCIATES, INC.

120 Pershing Street, P.O. Box 4526, East Providence, RI 02914

Russell F. Geisser Phone: 401-438-7320

Accreditation Renewal Date: January 1, 1986

$\begin{array}{ll}\text { NVLAP Code } & \text { Short Title } \\ & \text { PHYSICAL/FIRE TEST GROUP (04/F00) } \\ \text { 04/F01 } & \text { Test Installation } \\ \text { 04/F02 } & \text { Temperature Measurement } \\ \text { 04/F04 } & \text { Radiant Fire Test } \\ \text { 04/F05 } & \text { Coal Fire Test } \\ \text { 04/F06 } & \text { Brand Fire Test } \\ \text { 04/F07 } & \text { Flash Fire Test } \\ \text { 04/F08 } & \text { Strength Tests } \\ \text { 04/F09 } & \text { Stability Test } \\ \text { 04/F10 } & \text { Glazing Test } \\ & \text { MOBILE HOME TEST GROUP (04/M00) } \\ \text { 04/M01 } & \text { Test Installation } \\ \text { 04/M02 } & \text { Toxic Gas } \\ \text { 04/M03 } & \text { Drop Test }\end{array}$

Section of UL 737 Section of UL 1482

5th Edition 2nd Edition (March 1, 1982) (January 24, 1983)

PHYSICAL/FIRE TEST GROUP (04/F00)

Test Installation

Temperature Measurement

$\begin{array}{rr}8 & 8 \\ 9 & 9 \\ 11 & 11 \\ & 14 \\ 12 & 12 \\ 13 & 13 \\ 15 & 16 \\ 16 & 16 \\ 14 & 15\end{array}$




$\begin{array}{ll}\text { NVLAP Code } & \text { Short Title } \\ & \text { ELECTRICAL TEST GROUP (04/E00) } \\ \text { 04/E01 } & \text { Test Voltages } \\ \text { 04/E02 } & \text { Temperature Measurements, Electrical Components } \\ \text { 04/E03 } & \text { Input Test } \\ \text { 04/E04 } & \text { Temperature Test, Electrical Components } \\ \text { 04/E05 } & \text { Leakage Current } \\ \text { 04/E06 } & \text { Dielectric Withstand } \\ \text { 04/E07 } & \text { Locked Rotor (Stalled Motor) Temperature } \\ \text { 04/E08 } & \text { Power Cord Strain Relief }\end{array}$

Section of UL 737

5th Edition

(March 1, 1982)

Section of UL 1482

2nd Edition

(January 24, 1983)

NVLAP LAB CODE 0246

STOVE TESTING LAB

2721 North Hayden Island Drive, Portland, OR 97217

Sharon Conrad

Phone: 503-283-9711

Accreditation Renewal Date: January 1, 1986

\begin{tabular}{|c|c|}
\hline NVLAP Code & $\begin{array}{l}\text { Short Title } \\
\text { PHYSICAL/FIRE TEST GROUP (04/F00) }\end{array}$ \\
\hline 04/F01 & Test Installation \\
\hline 04/F02 & Temperature Measurement \\
\hline 04/F04 & Radiant Fire Test \\
\hline 04/F05 & Coal Fire Test \\
\hline 04/F06 & Brand Fire Test \\
\hline 04/F07 & Flash Fire Test \\
\hline 04/F08 & Strength Tests \\
\hline 04/F09 & Stability Test \\
\hline $04 / F 10$ & Glazing Test \\
\hline & MOBILE HOME TEST GROUP (04/M00) \\
\hline 04/M01 & Test Installation \\
\hline 04/M02 & Toxic Gas \\
\hline 04/M03 & Drop Test \\
\hline & ELECTRICAL TEST GROUP (04/E00) \\
\hline 04/E01 & Test Voltages \\
\hline 04/E02 & Temperature Measurements, Electrical Components \\
\hline $04 / \mathrm{E} 03$ & Input Test \\
\hline 04/E04 & Temperature Test, Electrical Components \\
\hline 04/E05 & Leakage Current \\
\hline 04/E06 & Dielectric Withstand \\
\hline 04/E07 & Locked Rotor (Stalled Motor) Temperature \\
\hline 04/E08 & Power Cord Strain Relief \\
\hline
\end{tabular}
Section of UL 737 Section of UL 1482
5th Edition 2nd Edition
(March 1, 1982) (January 24, 1983)

PHYSICAL/FIRE TEST GROUP (04/F00)

$\begin{array}{ll}33 & 33 \\ 34 & 34 \\ 35 & 35 \\ 36 & 36 \\ 38 & 38 \\ 37 & 37 \\ 39 & 39 \\ 40 & 40\end{array}$

$\begin{array}{rr}8 & 8 \\ 9 & 9 \\ 11 & 11 \\ & 14 \\ 12 & 12 \\ 13 & 13 \\ 15 & 16 \\ 16 & 16 \\ 14 & 15 \\ & \\ 17 & 17 \\ 17 & 17 \\ 17 & 17 \\ & \\ 33 & 33 \\ 34 & 34 \\ 35 & 35 \\ 36 & 36 \\ 38 & 38 \\ 37 & 37 \\ 39 & 39 \\ 40 & 40\end{array}$

NVLAP LAB CODE 0247

HOLLYTEX CARPET MILLS

505 N.E. Seventh Street, Anadarko, OK 73005

Chet Link Phone: 405-247-6641

Accreditation Renewal Date: April 1, 1985

$\begin{array}{ll}\text { NVLAP Code } & \text { Designation } \\ 03 / \mathrm{C} 02 & \text { AATCC } 8\end{array}$
Short Title

Colorfastness to Crocking 


$\begin{array}{lll}\text { NVLAP Code } & \begin{array}{l}\text { Designation } \\ \text { 03/S01 }\end{array} & \begin{array}{l}\text { Short Title } \\ \text { TSTM D1335 }\end{array} \\ & \begin{array}{l}\text { Federal Test Method } \\ \text { Standard 191-5100 }\end{array} & \\ & \text { Textile Test Method - Breaking Strength } \\ & \text { Tex } & \text { Textile Test Method - Delamination } \\ \text { 03/F03 } & \text { DoC FF 1-70 } & \text { Methenamine Pill Test }\end{array}$

NVLAP LAB CODE 0248

\title{
KNAUF FIBER GLASS RESEARCH LABORATORIES
}

240 Elizabeth Street, Shelbyville, IN 46176

Kerry Van Arsdel Phone: 317-398-4434

Accreditation Renewal Date: April 1, 1985

$\begin{array}{ll}\text { NVLAP Code } & \begin{array}{l}\text { Designation } \\ 01 / \text { D02 }\end{array} \\ \text { ASTM C167 } \\ 01 / \text { D08 } & \text { ASTM C302 } \\ 01 / \text { D09 } & \text { ASTM C303 } \\ 01 / \text { D11 } & \text { ASTM C356 } \\ & \\ 01 / \text { D12 } & \text { ASTM C411 } \\ 01 / \text { D13 } & \text { ASTM C519 } \\ 01 / \text { S01 } & \text { ASTM C165 } \\ 01 / \text { T01 } & \text { ASTM C177 } \\ 01 / \text { T05 } & \text { ASTM C335 } \\ 01 / \text { T06 } & \text { ASTM C518 } \\ 01 / \text { T09 } & \text { ASTM C653 } \\ 01 / \text { T10 } & \text { ASTM C687 }\end{array}$

\author{
Short Title \\ Thickness and density; Blanket and batt \\ Density; Preformed pipe insulation \\ Density; Preformed block insulation \\ Linear shrinkage; Soaking heat; Preformed high temperature \\ insulation \\ Hot-surface performance; High temperature insulation \\ Density; Loose-fill (fibrous) \\ Compressive properties; Thermal insulation (proc. A) \\ Thermal transmission properties; Low-temperature guarded hot plate \\ Thermal conductivity; Pipe insulation \\ Thermal transmission properties; Heat flow meter \\ Thermal resistance (Rec. Practice); Blanket (mineral fiber) \\ Thermal resistance (Rec. Practice); Loose-fill (fibrous)
}

NVLAP LAB CODE 0249

\section{WARNOCK HERSEY INTERNATIONAL, INC. 8612 Fairway Place, Middleton, WI 53562 \\ James J. Husom \\ Phone: 608-836-4400}

Accreditation Renewal Date: January 1, 1986

$\begin{array}{llcc}\text { NVLAP Code } & \text { Short Title } & \begin{array}{c}\text { 5th Edition } \\ \text { (March 1, 1982) }\end{array} & \begin{array}{c}\text { 2nd Edition } \\ \text { (January 24, 1983) }\end{array} \\ & \text { PHYSICAL/FIRE TEST GROUP (04/F00) } & 8 & 8 \\ \text { 04/F01 } & \text { Test Installation } & 9 & 9 \\ \text { 04/F02 } & \text { Temperature Measurement } & 11 & 11 \\ \text { 04/F04 } & \text { Radiant Fire Test } & & 14 \\ \text { 04/F05 } & \text { Coal Fire Test } & 12 & 12 \\ \text { 04/F06 } & \text { Brand Fire Test } & 13 & 13 \\ \text { 04/F07 } & \text { Flash Fire Test } & 15 & 16 \\ \text { 04/F08 } & \text { Strength Tests } & 16 & 16 \\ \text { 04/F09 } & \text { Stability Test } & 14 & 15 \\ \text { 04/F10 } & \text { Glazing Test } & & \\ & \text { MOBILE HOME TEST GROUP (04/M00) } & 17 & 17 \\ \text { 04/M01 } & \text { Test Installation } & 17 & 17 \\ 04 / \text { M02 } & \text { Toxic Gas } & 17 & \\ 04 / \text { M03 } & \text { Drop Test } & & 33 \\ & \text { ELECTRICAL TEST GROUP (04/E00) } & 33 & 34 \\ 04 / \text { E01 } & \text { Test Voltages } & 34 & \end{array}$


NVLAP Code Short Title

04/E03 Input Test

04/E04 Temperature Test, Electrical Components

04/E05

04/E06

04/E07

04/E08
Leakage Current

Dielectric Withstand

Locked Rotor (Stalled Motor) Temperature

Power Cord Strain Relief
Section of UL 737

5th Edition

(March 1, 1982)

35

36

38

37

39

40
Secrion of UL 1482

2nd Edition

(January 24, 1983)

35

36

38

37

39

40

NVLAP LAB CODE 0250

W. R. GRACE \& COMPANY

THERMAL PRODUCTS LABORATORY

62 Whittemore Avenue, Cambridge, MA 02140

Gregory Derderian Phone: 617-876-1400

Accreditation Renewal Date: April 1, 1985

NVLAP Code

01/D09

$01 / \mathrm{D} 14$

01/T04

01/T06
Designation

ASTM C303

ASTM C520

ASTM C236

ASTM C518
Short Title

Density; Preformed block insulation

Density; Granular loose-fill

Thermal conductance; Guarded hot box

Thermal transmission properties; Heat flow meter

NVLAP LAB CODE 0251

\section{STATE OF CALIFORNIA \\ BUREAU OF HOME FURNISHINGS \\ 3485 Orange Grove Avenue, North Highlands, CA 95660 \\ John A. McCormack Phone: 916-920-6952}

Accreditation Renewal Date: July 1, 1985

$\begin{array}{lll}\text { NVLAP Code } & \begin{array}{l}\text { Designation } \\ \text { 01/F07 }\end{array} & \begin{array}{l}\text { Short Title } \\ \text { HH-I-515 } \\ \text { (para. 4.8.7 in D version, }\end{array} \\ & \begin{array}{l}\text { Amendment 1) } \\ \text { 01/F08 }\end{array} & \begin{array}{l}\text { HH-I-515 } \\ \text { (para. 4.8.8 in D version, }\end{array} \\ & \text { Amendment 1) } & \\ & \end{array}$

NVLAP LAB CODE 0252

\section{D/L LABORATORIES}

116 East 16th Street, New York, NY 10003

Saul Spindel Phone: 212-777-4410

Accreditation Renewal Date: October 1, 1985

Paints and Related Coatings and Materials

NVLAP Code Designation

Measurements of Intrinsic Physical Properties

09/A01

$09 / \mathrm{A} 02$

$09 / \mathrm{A} 04$

09/A05

09/A07

09/A08
ASTM D56

ASTM D93

ASTM D185

ASTM D281

ASTM D523

ASTM D562
Short Title

Flash Point by Tag Closed Tester

Flash Point by Pensky-Martens Closed Tester, Method A \& B

Coarse Particles in Pigments, Pastes and Paints

Oil Absorption of Pigments by Spatula Rub-Out

Specular Gloss

Consistency of Paints Using the Stormer Viscometer

Procedure A \& B 


$\begin{array}{ll}\text { NVLAP Code } & \begin{array}{l}\text { Designation } \\ \text { 09/A10 }\end{array} \\ \text { ASTM D1186 } \\ \text { 09/A11 } & \text { ASTM D1200 } \\ \text { 09/A12 } & \text { ASTM D1210 } \\ \text { 09/A13 } & \text { ASTM D1212 } \\ \text { 09/A14 } & \text { ASTM D1296 } \\ \text { 09/A15 } & \text { ASTM D1310 } \\ \text { 09/A16 } & \text { ASTM D1400 } \\ \text { 09/A17 } & \text { ASTM D1475 } \\ \text { 09/A18 } & \text { ASTM D1544 } \\ \text { 09/A20 } & \text { ASTM D2244 } \\ \text { 09/A21 } & \text { ASTM D3278 } \\ \text { 09/A22 } & \text { ASTM D3363 } \\ \text { 09/A25 } & \text { ASTM D4212 } \\ \text { 09/A26 } & \text { ASTM E97 } \\ & \\ \text { 09/A28 } & \text { ASTM E313 }\end{array}$

Short Title

Dry Film Thickness of Non-magnetic Coatings Applied to a Ferrous Base, Method A \& B

Viscosity of Paints, Varnishes, and Lacquers by Ford Viscosity Cup

Fineness of Dispersion of Pigment-Vehicle Systems

Wet Film Thickness of Organic Coatings, Method A

Odor of Volatile Solvents and Diluents

Flash-Point of Liquids by Tag Open-Cup Apparatus

Dry Film Thickness of Non-conductive Coatings Applied to a Nonferrous Metal Base

Density of Paint, Varnish, Lacquer, and Related Products

Color of Transparent Liquids (Gardner Color Scale)

Instrumental Evaluation of Color Difference of Opaque Materials

Flash Point of Liquids by Setaflash Closed Tester Method A \& B

Film Hardness by Pencil Test

Viscosity by Dip-Type Viscosity Cups

45- deg, 0-deg Directional Reflectance Factor of Opaque

Specimens by Broad-Band Filter Reflectometry

Indexes of Whiteness and Yellowness of Near-White Opaque Materials

Measurements of Performance and Performance Change

09/B01

09/B02

09/B03

09/B04

09/B05

09/B06

09/B07

09/B08

09/B09

$09 / \mathrm{B} 10$

$09 / \mathrm{B} 11$

$09 / \mathrm{B} 12$

$09 / \mathrm{B} 13$

$09 / \mathrm{B} 14$

$09 / \mathrm{B} 15$

$09 / \mathrm{B} 16$

$09 / \mathrm{B} 17$

09/B18

$09 / \mathrm{B} 19$

09/B20

$09 / \mathrm{B} 23$

09/B24

09/B25

09/B26

$09 / \mathrm{B} 27$

09/B29

09/B30

09/B31

09/B32

09/B33

ASTM D3274

09/B34
ASTM D279

ASTM D332

ASTM D344

ASTM D610

ASTM D659

ASTM D660

ASTM D661

ASTM D662

ASTM D711

ASTM D714

ASTM D772

ASTM D821

ASTM D868

ASTM D869

ASTM D870

ASTM D913

ASTM D968

ASTM D969

ASTM D1308

ASTM D1309

ASTM D1640

ASTM D1737

ASTM D2197

ASTM D2243

ASTM D2248

ASTM D2486

ASTM D2801

ASTM D2805

ASTM D3273

ASTM D3450
Bleeding of Pigments, Method A \& B

Tinting Strength of White Pigments, Method A

Relative Dry Hiding Power of Paints

Rusting on Painted Steel Surfaces

Chalking of Exterior Paints

Checking of Exterior Paints

Cracking of Exterior Paints

Erosion of Exterior Paints

No-Pick-Up Time of Traffic Paint

Blistering of Paints

Flaking (Scaling) of Exterior Paints

Abrasion, Erosion or a Combination of Both in Road Service

Tests of Traffic Paints

Bleeding of Traffic Paint

Settling of Traffic Paint

Water Immersion Test of Organic Coatings on Steel

Chipping of Traffic Paint

Abrasion Resistance of Organic Coatings by the Falling

Abrasive Tester, Method A \& B

Bleeding of Traffic Paint

Effect of Household Chemicals on Clear and

Settling Properties of Traffic Paint During

Drying, Curing, or Film Formation of Organic

Elongation of Attached Organic Coatings with Cylindrical

Mandrel Apparatus

Adhesion of Organic Coatings, Method A

Freeze-Thaw Resistance of Latex and Emulsion Paints

Detergent Resistance of Organic Finishes

Scrub Resistance of Interior Latex Flat Wall Paints

Leveling Characteristics of Paints by Draw-Down Method

Hiding Power of Paints

Resistance to Growth of Mold on the Surface of Interior Coatings in an Environmental Chamber

Surface Disfigurement of Paint Films by Fungal Growth or

Soil and Dirt Accumulation

Washability Properties of Interior Architectural Coatings 


$\begin{array}{ll}\text { NVLAP Code } & \text { Designation } \\ \text { 09/B35 } & \text { ASTM D3456 } \\ \text { 09/B37 } & \text { ASTM D4060 } \\ \text { 09/B38 } & \text { ASTM D4062 } \\ \text { 09/B39 } & \text { ASTM D4213. } \\ \text { 09/B40 } & \text { ASTM D4214 } \\ \text { 09/B41 } & \text { Fed. Std. 141 } \\ \text { 09/B42 } & \text { Fed. Std. } 141\end{array}$

Short Title

Susceptability of Paint Films to Microbioligical Attack

Abrasion Resistance of Organic Coatings by the Taber Abraser

Leveling of Paints by Draw-Down Method

Wet Abrasion Resistance of Interior Paint by Weight Loss

Chalking of Exterior Paint Films, Method A, B, C, D \& E

Sag Test (Multinotch Blade) Method 4494

Drying Time Method 4061

Measurement of Chemical Properties and Compositions
09/C09
ASTM D 1259
$09 / \mathrm{C} 12$
ASTM D1364
$09 / \mathrm{C} 26$
ASTM D2369
$09 / \mathrm{C} 27$
ASTM D2371
$09 / \mathrm{C} 28$
ASTM D2697
09/C29
ASTM D2698
$09 / \mathrm{C} 30$
ASTM D2832
09/C37
ASTM D3723
09/C39
ASTM D3960
$09 / \mathrm{C} 40$
ASTM D4017
Nonvolatile Content of Resin Solutions, Method A \& B
Water in Volatile Solvents (Fischer Reagent Titration Method)
Volatile Content of Paints, Procedure A \& B
Pigment Content of Solvent-Type Paints
Volume Nonvolatile Matter in Clear or Pigmented Coatings
Pigment Content Of Solvent-Type Paints by High-Speed Centrifuging
Nonvolatile Content of Paint and Paint Materials
Pigment Content of Water-Emulsion Paints by
Low-Temperature Ashing
Volatile Organic Contents (VOC) of Paints and Related Coatings
Water in Paints and Paint Materials by Karl Fischer Method

Test Sample Conditioning and Preparation

09/D01 ASTM B117

09/D02 ASTM D609

09/D03 ASTM D822

09/D04 ASTM D823

09/D05 ASTM D1006

09/D06 ASTM D1014

09/D10 ASTM D2247

09/D11 ASTM D2372

09/D13 ASTM D3924

09/D14 ASTM G23

09/D16 ASTM G53

\section{Salt Spray (Fog) Testing}

Preparation of Steel Panels for Testing Paints Varnish, Lacquer, and Related Products, Method A, B, C, \& D

Operating Light-and-Water-Exposure Apparatus

(Carbon-Arc Type) for Testing Paint, Varnish, Lacquer, and Related Products

Producing Films of Uniform Thickness of Paint Varnish, Lacquer, and Related Products on Test Panels, Method B \& D

Exterior Exposure Tests of Paints on Wood

Exterior Exposure Tests of Paints on Steel, Method A, B, D, E, \& F

Coated Metal Specimens at 100\% Relative Humidity

Separation of Vehicle Solvent-Type Paints

Standard Environment for Conditioning and Testing Paint,

Varnish, Lacquer, and Related Materials

Operating Light-Exposure Apparatus (Carbon-Arc Type)

With and Without Water for Exposure of Nonmetallic

Materials, Method 1, 2, 3, \& 4

Operating Light- and Water-Exposure Apparatus (Fluorescent UV-

Condensation Type) for Exposure of Nonmetallic Materials

NVLAP LAB CODE 0253

\section{GIFFORD-HILL AND COMPANY, INC. TECHNICAL SERVICES DIVISION LABORATORY \\ 240 Singleton Blvd., P.O. Box 47127, Dallas, TX 75247 \\ K. Stuart Pryor, II Phone: 214-651-0066}

Accreditation Renewal Date: October 1, 1985

$\begin{array}{ll}\text { NVLAP Code } & \text { Designation } \\ \text { 02/M01 } & \text { ASTM C31 } \\ \text { 02/M03 } & \text { ASTM C172 } \\ \text { 02/P01 } & \text { ASTM C143 } \\ \text { 02/W01 } & \text { ASTM C138 } \\ \text { 02/A01 } & \text { ASTM C231 }\end{array}$

Short Title

Making and Curing Concrete Test Specimens in the Field

Sampling Fresh Concrete

Slump of Portland Cement Concrete

Unit Weight, Yield, and Air Content (Gravimetric) of Concrete

Air Content of Freshly Mixed Concrete by the Pressure Method 


$\begin{array}{ll}\text { NVLAP Code } & \text { Designation } \\ \text { 02/S01 } & \text { ASTM C39 } \\ \text { 02/A02 } & \text { ASTM C173 }\end{array}$

\section{Short Title}

Compressive Strength of Cylindrical Concrete Specimens

Air Content of Freshly Mixed Concrete by the Volumetric Method

\section{NVLAP LAB CODE 0254}

\section{CHEMRAY COATINGS CORP. 150 Lincoln Blvd., Middlesex, NJ 08846}

Frederick W. Armstrong, Jr. Phone: 201-469-1110

Accreditation Renewal Date: October 1, 1985

Paints and Related Coatings and Materials

$\begin{array}{ll}\text { NVLAP Code } & \begin{array}{c}\text { Designation } \\ \text { Measurements of Intrinsic Physical Properties } \\ \text { 09/A02 }\end{array} \\ \text { 09/A03 } & \text { ASTM D93 } \\ \text { 09/A04 } & \text { ASTM D153 } \\ \text { 09/A05 } & \text { ASTM D185 } \\ \text { 09/A07 } & \text { ASTM D523 } \\ \text { 09/A08 } & \text { ASTM D562 } \\ \text { 09/A09 } & \text { ASTM D1005 } \\ \text { 09/A10 } & \text { ASTM D1186 } \\ & \\ \text { 09/A11 } & \text { ASTM D1200 } \\ \text { 09/A12 } & \text { ASTM D1210 } \\ \text { 09/A13 } & \text { ASTM D1212 } \\ \text { 09/A14 } & \text { ASTM D1296 } \\ \text { 09/A17 } & \text { ASTM D1475 } \\ \text { 09/A18 } & \text { ASTM D1544 } \\ \text { 09/A19 } & \text { ASTM D1729 } \\ \text { 09/A20 } & \text { ASTM D2244 } \\ \text { 09/A21 } & \text { ASTM D3278 } \\ \text { 09/A22 } & \text { ASTM D3363 } \\ \text { 09/A25 } & \text { ASTM D4212 } \\ \text { 09/A26 } & \text { ASTM E97 } \\ & \\ \text { 09/A27 } & \text { ASTM E308 } \\ \text { 09/A28 } & \text { ASTM E313 }\end{array}$

\section{Short Title}

Flash Point by Pensky-Martens Closed Tester, Method A \& B

Specific Gravity of Pigments

Coarse Particles in Pigments, Pastes and Paints

Oil Absorption of Pigments by Spatula Rub-Out

Specular Gloss

Consistency of Paints Using the Stormer Viscometer

Procedure A \& B

Dry Film Thickness of Organic Coatings

Dry Film Thickness of Non-magnetic Coatings Applied to a Ferrous Base, Method A \& B

Viscosity of Paints, Varnishes, and Lacquers by Ford Viscosity Cup

Fineness of Dispersion of Pigment-Vehicle Systems

Wet Film Thickness of Organic Coatings, Method A

Odor of Volatile Solvents and Diluents

Density of Paint, Varnish, Lacquer, and Related Products

Color of Transparent Liquids (Gardner Color Scale)

Visual Evaluation of Color Differences of Opaque Materials

Instrumental Evaluation of Color Difference of Opaque Materials

Flash Point of Liquids by Setaflash Closed Tester, Method A \& B

Film Hardness by Pencil Test

Viscosity by Dip-Type Viscosity Cups

45- deg, 0-deg Directional Reflectance Factor of Opaque

Specimens by Broad-Band Filter Reflectometry

Spectrophotometry and Description of Color in CIE 1931 System

Indexes of Whiteness and Yellowness of Near-White Opaque

Materials

Measurements of Performance and Performance Change

$\begin{array}{ll}\text { 09/B02 } & \text { ASTM D332 } \\ \text { 09/B03 } & \text { ASTM D344 } \\ \text { 09/B05 } & \text { ASTM D659 } \\ \text { 09/B09 } & \text { ASTM D711 } \\ \text { 09/B10 } & \text { ASTM D714 } \\ \text { 09/B11 } & \text { ASTM D772 } \\ \text { 09/B13 } & \text { ASTM D868 } \\ \text { 09/B14 } & \text { ASTM D869 } \\ \text { 09/B15 } & \text { ASTM D870 } \\ \text { 09/B16 } & \text { ASTM D913 } \\ \text { 09/B17 } & \text { ASTM D968 } \\ & \\ \text { 09/B18 } & \text { ASTM D969 } \\ \text { 09/B20 } & \text { ASTM D1309 } \\ \text { 09/B23 } & \text { ASTM D1640 }\end{array}$

Tinting Strength of White Pigments, Method A

Relative Dry Hiding Power of Paints

Chalking of Exterior Paints

No-Pick-Up Time of Traffic Paint

Blistering of Paints

Flaking (Scaling) of Exterior Paints

Bleeding of Traffic Paint

Settling of Traffic Paint

Water Immersion Test of Organic Coatings on Steel

Chipping of Traffic Paint

Abrasion Resistance of Organic Coatings by the Falling

Abrasive Tester, Method A \& B

Bleeding of Traffic Paint

Settling Properties of Traffic Paint During

Drying, Curing, or Film Formation of Organic 


$\begin{array}{ll}\begin{array}{l}\text { NVLAP Code } \\ \text { 09/B24 }\end{array} & \begin{array}{l}\text { Designation } \\ \text { ASTM D1737 }\end{array} \\ \text { 09/B25 } & \text { ASTM D2197 } \\ \text { 09/B26 } & \text { ASTM D2243 } \\ \text { 09/B29 } & \text { ASTM D2486 } \\ \text { 09/B30 } & \text { ASTM D2801 } \\ \text { 09/B31 } & \text { ASTM D2805 } \\ \text { 09/B34 } & \text { ASTM D3450 } \\ \text { 09/B38 } & \text { ASTM D4062 } \\ \text { 09/B41 } & \text { Fed. Std. } 141 \\ \text { 09/B42 } & \text { Fed. Std. } 141\end{array}$

\section{Short Title}

Elongation of Attached Organic Coatings with Cylindrical

Mandrel Apparatus

Adhesion of Organic Coatings, Method A

Freeze-Thaw Resistance of Latex and Emulsion Paints

Scrub Resistance of Interior Latex Flat Wall Paints

Leveling Characteristics of Paints by Draw-Down Method

Hiding Power of Paints

Washability Properties of Interior Architectural Coatings

Leveling of Paints by Draw-Down Method

Sag Test (Multinotch Blade) Method 4494

Drying Time Method 4061

Measurement of Chemical Properties and Compositions

09/C01 ASTM D34

09/C02 ASTM D95

$09 / \mathrm{CO4}$

ASTM D563

09/C06

ASTM D 1078

$09 / \mathrm{C} 07$

ASTM D1133

09/C08

09/C09

09/C10

ASTM D 1208

ASTM D 1259

ASTM D1306

09/C11

ASTM D1353

$09 / \mathrm{C} 12$

$09 / \mathrm{C} 1 \hat{3}$

$09 / \mathrm{C} 14$

$09 / \mathrm{C} 15$

09/C17

$09 / \mathrm{C} 19$

$09 / \mathrm{C} 21$

$09 / \mathrm{C} 22$

$09 / \mathrm{C} 26$

$09 / \mathrm{C} 28$

09/C29

09/C30

$09 / \mathrm{C} 31$

09/C 32

09/C33

09/C34

ASTM D3335

09/C39

ASTM D3960

Test Sample Conditioning and Preparation

09/D01

09/D02

ASTM B117

ASTM D609

09/D03

ASTM D822

09/D04

ASTM D823

09/D08
Chemical Analysis of White Pigments Pigments

Water in Petroleum Products and Bituminous Materials by Distillation

Phthalic Anhydride Content of Alkyd Resins and Resin Solutions

Distillation Range of Volatile Organic Liquids

Kauri-Butanol Value of Hydro-carbon Solvents

Common Properties of Certain Pigments

Nonvolatile Content of Resin Solutions, Method A \& B

Phthalic Anhydride Content of Alkyd Resins and Esters Containing Other Dibasic Acids (Gravimetric)

Nonvolatile Matter in Volatile Solvents for Use in Paint, Varnish, Lacquer and Related Products

Water in Volatile Solvents (Fischer Reagent Titration Method)

Chemical Analysis of White Titanium Pigments

Unsaponifiable Matter in Alkyd Resins and Resins Solutions

Fatty Acid Content of Alkyd Resins and Alkyd Resin

Solutions, Niethod A \& B

Fatty Acids Used in Protective Coatings

Total Iodine Value of Drying Oils and Their Derivatives

Acid Value of Organic Coating Materials

Nonvolatile Content of Varnishes, Method A \& B

Volatile Content of Paints, Procedure A \& B

Volume Nonvolatile Matter in Clear or Pigmented Coatings

Pigment Content Of Solvent-Type Paints by High-Speed

Centrifuging

Nonvolatile Content of Paint and Paint Materials

Composition of Turpentine by Gas Chromatography

Direct Injection of Solvent-Base Paints into a Gas

Chromatograph for Solvent Analysis

Vacuum Distillation of Solvents from Solvent-Base Paints for Analysis

Low Concentrations of Lead, Cadmium, and Cobalt in Paint by Atomic Absorption Spectroscopy

Volatile Organic Contents (VOC) of Paints and Related Coatings

Salt Spray (Fog) Testing

Preparation of Steel Panels for Testing Paints Varnish, Lacquer, and Related Products, Method A, B, C, \& D

Operating Light-and-Water-Exposure Apparatus (Carbon-Arc Type) for Testing Paint, Varnish, Lacquer, and Related Products

Producing Films of Uniform Thickness of Paint Varnish, Lacquer, and Related Products on Test Panels, Method B, C, \& D

Preparation of Aluminum and Aluminum-Alloy Surfaces for Painting, Type A, B, C, \& D 


$\begin{array}{ll}\begin{array}{l}\text { NVLAP Code } \\ \text { 09/D09 }\end{array} & \begin{array}{l}\text { Designation } \\ \text { ASTM D1734 }\end{array} \\ \text { 09/D11 } & \text { ASTM D2372 } \\ \text { 09/D13 } & \text { ASTM D3924 } \\ \text { 09/D14 } & \text { ASTM G23 } \\ & \\ \text { 09/D16 } & \text { ASTM G53 }\end{array}$
Short Title
Making and Preparing Concrete and Masonry Panels for Testing Paint Finishes
Separation of Vehicle Solvent-Type Paints
Standard Environment for Conditioning and Testing Paint,
Varnish, Lacquer, and Related Materials
Operating Light-Exposure Apparatus (Carbon-Arc Type)
With and Without Water for Exposure of Nonmetallic
Materials, Method 1, 2, 3, \& 4
Operating Light- and Water-Exposure Apparatus (Fluorescent UV-
Condensation Type) for Exposure of Nonmetallic Materials

NVLAP LAB CODE 0255

\section{UNDERWRITERS LABORATORIES INC.}

1285 Walt Whitman Road, Melville, NY 11747

R. W. Miller Phone: 516-271-6200

Accreditation Renewal Date: October 1, 1985

$\begin{array}{lll}\text { NVLAP Code } & \text { Designation } & \text { Short Title } \\ \text { 03/F03 } & \text { DoC FF 1-70 } & \text { Methenamine Pill Test } \\ \text { 03/F04 } & \text { ASTM E648 } & \text { Radiant Panel (Carpet) }\end{array}$

NVLAP LAB CODE 0501

\section{BALTIMORE GAS \& ELECTRIC COMPANY, CALVERT CLIFFS NUCLEAR POWER PLANT NUCLEAR POWER DEPARTMENT, DOSIMETRY UNIT \\ RADIATION SAFETY SECTION \\ Lusby, MD 20657 \\ Eugene T. Reimer \\ Phone: 301-269-4716}

Accreditation Renewal Date: October 1, 1986

This facility has been evaluated and deemed competent to process the radiation dosimeter listed below through employing a Panasonic Automatic reader model UD710A and Panasonic Manual reader UD702A.

This facility is accredited to process the following dosimeter by virtue of actual demonstration of compliance with ANSI-N13.11-1983 through testing.

Panasonic TLD model UD802 for ANSI-N13.11 categories I, II, III, IV, V, VI, VII, VIII.

NVLAP LAB CODE 0503

\section{MALLINCKRODT DIAGNOSTICS, INC. \\ 2703 Wagner Place, Maryland Heights, MO 63043 \\ Mark Doruff Phone: 314-344-3981}

Accreditation Renewal Date: October 1, 1986

This facility has been evaluated and deemed competent to process the radiation dosimeter listed below through employing a Harshaw Automatic readers model 2000B and 2000D.

This facility is accredited to process the following dosimeter by virtue of actual demonstration of compliance with ANSI-N13.11-1983 through testing.

Harshaw TLD model 100 for ANSI-N13.11 category VII. 


\section{NAVAL MEDICAL COMMAND \\ NATIONAL CAPITAL REGION \\ RADIATION SAFETY DEPARTMENT \\ Bethesda, MD 20814 \\ Eric E. Kearsley \\ Phone: 202-295-5414}

Accreditation Renewal Date: October 1, 1986

This facility has been evaluated and deemed competent to process the radiation dosimeters listed below through employing a Harshaw Automatic reader model 2271 and Manual film processing using a Macbeth densitometer.

This facility is accredited to process the following dosimeter by virtue of actual demonstration of compliance with ANSI-N13.11-1983 through testing.

Harshaw TLD Albedo (1 TLD 600, 1 TLD 700) for ANSI-N13.11 Categories II, IV, VIII.

Film Badge (Kodak Type 3) for ANSI-N13.11 Categories II, III, IV, V, VI, VII.

NVLAP LAB CODE 0506

\section{SOUTHERN CALIFORNIA EDISON SAN ONOFRE NUCLEAR GENERATING STATION}

P.O. Box 128, San Clemente, CA 92672

Kathryn H. Swoope

Phone: 714-492-7700

Accreditation Renewal Date: October 1, 1986

This facility has been evaluated and deemed competent to process the radiation dosimeter listed below through employing a Panasonic Automatic reader model UD710A.

This facility is accredited to process the following dosimeter by virtue of actual demonstration of compliance with ANSI-N13.11-1983 through testing.

Panasonic TLD model UD802-AS2 for ANSI-N13.11 categories I, II, III, IV, V, VI, VII.

NVLAP LAB CODE 0507

\section{U.S. ENVIRONMENTAL PROTECTION AGENCY NUCLEAR RADIATION ASSESSMENT DIVISION}

P.O. Box 15027, Las Vegas, NV 89114

Jaci L. Hopper Phone: 702-798-2320

Accreditation Renewal Date: October 1, 1986

This facility has been evaluated and deemed competent to process the radiation dosimeter listed below through employing a Harshaw Automatic reader model 2271.

This facility is accredited to process the following dosimeter by virtue of actual demonstration of compliance with ANSI-N13.11-1983 through testing.

Harshaw TLD Model TL-200 for ANSI-N13.11 categories II, IV. 


\section{NEW YORK POWER AUTHORITY \\ INDIAN POINT UNIT NO. 3 NUCLEAR POWER PLANT \\ P.O. Box 215, Buchanan, NY 10511 \\ Thomas Labenski Phone: 914-739-8200}

Accreditation Renewal Date: October 1, 1986

This facility has been evaluated and deemed competent to process the radiation dosimeter listed below through employing a Panasonic Automatic reader model UD710B and Panasonic Manual reader UD702E.

This facility is accredited to process the following dosimeter by virtue of actual demonstration of compliance with ANSI-N13.11-1983 through testing.

Panasonic TLD model UD806AQ for ANSI-N13.11 categories I, II, III, IV, V, VI, VII.

NVLAP LAB CODE 0509

NAVAL RESEARCH LABORATORY

Code 6073, Washington, DC 20375

Kirk J. King Phone: 202-767-2232

Accreditation Renewal Date: January 1, 1987

This facility has been evaluated and deemed competent to process the radiation dosimeter listed below through employing a Harshaw Automatic reader model 2271.

This facility is accredited to process the following dosimeter by virtue of actual demonstration of compliance with ANSI-N13.11-1983 through testing.

NRL Radiation Badge for ANSI-N13.11 categories II, III, IV, VI, VIII.

NVLAP LAB CODE 0510

\section{GENERAL PUBLIC UTILITIES NUCLEAR CORPORATION DIVISION OF RADIOLOGICAL \& ENVIRONMENTAL CONTROLS \\ Route 441 South, P.O. Box 480, Middletown, PA 17057 \\ O. Ronald Perry Phone: 717-948-8595}

Accreditation Renewal Date: October 1, 1986

This facility has been evaluated and deemed competent to process the radiation dosimeter listed below through employing a Panasonic Automatic reader model UD710.

This facility is accredited to process the following dosimeter by virtue of actual demonstration of compliance with ANSI-N13.11-1983 through testing.

Panasonic TLD model UD802-2 for ANSI-N13.11 categories I, II, III, IV, V, VI, VII, and Panasonic TLD model UD802-2N for ANSI-N13.11 categories IV, VIII. 


\section{NEW YORK POWER AUTHORITY \\ JAMES A. FITZPATRICK NUCLEAR POWER PLANT \\ P.O. Box 41, Lycoming, NY 13093 \\ Dr. David A. Dooley Phone: 315-342-3840}

\section{Accreditation Renewal Date: October 1, 1986}

This facility has been evaluated and deemed competent to process the radiation dosimeter listed below through employing a Panasonic Automatic reader model UD710A.

This facility is accredited to process the following dosimeter by virtue of actual demonstration of compliance with ANSI-N13.11-1983 through testing.

Panasonic TLD model UD801 for ANSI-N13.11 categories II, IV, VI, VII.

NVLAP LAB CODE 0512

\section{RADIATION DETECTION COMPANY \\ 162 Wolfe Road, P.O. Box 1414, Sunnyvale, CA 94088 \\ Richard H. Holden Phone: 408-735-8700}

\section{Accreditation Renewal Date: October 1, 1986}

This facility has been evaluated and deemed competent to process the radiation dosimeters listed below through employing (1) modified CON RAD readers; (2)Teledyne 7100 reader; (3) Teledyne 7300 reader; (4) Harshaw 3000 reader; (5)Victoreen 2800 reader; (6) by manual film processing and reading on a Macbeth TD502 densitometer; or (7) Tracketch, NTA manual optical readers.

This faciiity is accredited to process the following dosimeters by virtue of actual demonstration of compliance with ANSI-N13.11-1983 through testing.

$\begin{array}{llll}\text { Designation } & \text { Process } & \text { ANSI N13.11 } & \text { Categories } \\ \text { Hi Energy } & \text { TLD } & 1 & \text { III, IV } \\ \text { Beta } & \text { TLD } & 1,3^{*} & \text { V, VII } \\ \text { Lo Energy } & \text { TLD } & 1,3^{*} & \text { I, III, VI } \\ \text { TLD } & \text { Albedo } & 3^{*}, 6 & \text { VIII } \\ \text { Film } & \text { XBG } & 6 & \text { I, II, III, IV, V, VI, VII } \\ \text { Film } & \text { XBGN } & 6,7 & \text { VIII } \\ \text { Neutron } & \text { Tracketch } & 7 & \text { VIII }\end{array}$

* Processes listed above 2, 4, and 5 are considered functionally acceptable as substitutes which can be used in lieu of process 3 as listed above.

NVLAP LAB CODE 0515

\section{EBERLINE SERVICES DIVISION DOSIMETRY DEPARTMENT}

P.O. Box 2108, Santa Fe, NM 87501

Nels Johnson

Phone: 505-345-9931

\section{Accreditation Renewal Date: October 1, 1986}

This facility has been evaluated and deemed competent to process the radiation dosimeter listed below through employing a Eberline Manual reader TLR-6.

This facility is accredited to process the following dosimeter by virtue of actual demonstration of compliance with ANSI-N13.11-1983 through testing.

Eberline TLD ( 2 or 3 Harshaw TLD 100 chips) for ANSI-N13.11 categories I, II, III, IV, V, VI, VII, VIII. 


\title{
CAROLINA POWER \& LIGHT COMPANY \\ HARRIS ENERGY \& ENVIRONMENTAL CENTER \\ Route 1, Box 327, New Hill, NC 27562 \\ Stephen A. Browne \\ Phone: 919-362-3212
}

\section{Accreditation Renewal Date: October 1, 1986}

This facility has been evaluated and deemed competent to process the radiation dosimeter listed below through employing a Panasonic Automatic reader model UD710A and Panasonic Manual reader UD702E.

This facility is accredited to process the following dosimeter by virtue of actual demonstration of compliance with ANSI-N13.11-1983 through testing.

Panasonic TLD model UD802AQ for ANSI-N13.11 categories I, II, III, IV, V, VI, VII, VIII.

The following sites are included in the accreditation as sub-facilities of the above listed main facility. These subfacilities are accredited by virtue of using identical equipment and procedures as indicated above.

Robinson Nuclear Plant, Hartsville, South Carolina Brunswick Nuclear Plant, Southport, South Carolina

NVLAP LAB CODE 0518

\author{
R.S. LANDAUER JR. \& COMPANY \\ Glenwood Science Park, 2 Science Park, Glenwood, IL 60425 \\ Craig Yoder Phone: 312-755-7000
}

Accreditation Renewal Date: October 1, 1986

This facility has been evaluated and deemed competent to process the radiation dosimeters listed below through employing (1) automatic film reader Tech/Ops model 1; (2) Harshaw Atlas Hotgas reader; (3) Harshaw 2271 reader; (4) NTA/Polycarbonate /CR-39 manual optical readers; or (5) manual densitometers X-Rite, Tech/Ops model 301, Macbeth model TD504.

This facility is accredited to process the following dosimeter by virtue of actual demonstration of compliance with ANSI-N13.11-1983 through testing.

$\begin{array}{llll}\text { Landauer designation } & \text { Film Process } & \text { ANSI N13.11 } & \text { Category } \\ \text { G-Film } & \text { "GARDRAY" } & 1,5 & \text { I, II, III, IV, V, VI, VII } \\ \text { P-G } & \text { badge plus NTA } & 1,4,5 & \text { VIII } \\ \text { A-G } & \text { badge plus polycarbonate } & 1,4 & \text { VIII } \\ \text { TLD } & & & \\ \text { L-4 chip } & \text { "GARDRAY" } & 2 & \text { I, II, III, IV, V, VI, VII } \\ \text { D-3 Harshaw } & 700 \text { chips } & 3 & \text { II, IV, V, VII } \\ \text { I-Neutrak } & \text { ER } & 3,4 & \text { VIII }\end{array}$

The facility is accredited to process the following dosimeters which have been deemed functionally acceptable by virtue of using identical techniques and equipment to process combinations of elements demonstrated above.

Landauer designation
B-G badge
C-G badge
P-G badge plus
H-G badge plus
A-G badge plus
J-G badge plus
Y-G badge plus
R-G badge plus
Q-DEX-RAY

TLD

F-L badge plus

-L badge plus
Film Process

plus CR-39

plus CR-39 and Cadmium

NTA

NTA and Cadmium

polycarbonate

polycarbonate and Cadmium

Cadmium

ER

CR-39

polycarbonate

ANSI N13.11
$1,4,5$
$1,4,5$
$1,4,5$
$1,4,5$
$1,4,5$
$1,4,5$
$1,4,5$
$1,3,4,5$
$1,4,5$

Category

I through VIII

I through VIII

I, II, III, IV, V, VI, VII, VIII

I through VIII

I, II, III, IV, V, VI, VII, VIII

I through VII

I, III

I, II, III, IV, V, VI, VII, VIII

I, III

$\begin{array}{ll}2,4 & \text { I through VIII } \\ 2,4 & \text { I through VIII }\end{array}$


The following sites are included in the accreditation as sub-facilities of the above listed main facility.

The following sub-facilities are accredited to process the Landauer " $D$ " badge employing a Harshaw 2271 automatic TLD reader for ANSI N13.11 categories II, IV, V, VII which have been deemed functionally acceptable by virtue of using identical techniques and procedures as demonstrated above for the items specified.

R.S. Landauer, Jr. \& Company Nuclear Station System (NSS) sites at:

Boston Edison Company, Pilgrim Station, Plymouth, Massachusetts Alabama Power, Farley Nuclear Plant, Ashford, Alabama

The following sub-facilities are accredited to perform limited volume, emergency response processing employing either a Harshaw 3000 manual reader or manual film processing techniques for the following badges:

\begin{tabular}{|c|c|c|c|}
\hline G-Film & "GARDRAY" & ANSI N13.11 & Categories I, II, III, IV, V, VI, VII \\
\hline L-TLD 4 chip & "GARDRAY" & ANSI N13.11 & Categories I, II, III, IV, V, VI, VII \\
\hline T-TLD 2 chip & & ANSI N13.11 & Categories II, IV, V, VII \\
\hline
\end{tabular}

R. S. Landauer, Jr. \& Company Offices: El Sequndo, California; Houston, Texas; Burlington, Massachusetts; and East Brunswick, New Jersey.

NVLAP LAB CODE 0519

\section{HOUSTON LIGHTING \& POWER COMPANY, MANAGING PARTNER SOUTH TEXAS PROJECT ELECTRIC GENERATING STATION}

\section{P.O. Box 1700, Houston, TX 77059}

Gene R. Jarvela Phone: 512-972-3651

\section{Accreditation Renewal Date: October 1, 1986}

This facility has been evaluated and deemed competent to process the radiation dosimeter listed below through employing a Panasonic Automatic reader model UD710A.

This facility is accredited to process the following dosimeter by virtue of actual demonstration of compliance with ANSI-N13.11-1983 through testing.

Panasonic TLD Model UD801 for ANSI-N13.11 category IV.

NVLAP LAB CODE 0520

\section{VIRGINIA ELECTRIC AND POWER COMPANY NORTH ANNA POWER STATION \\ P.O. Box 402, Mineral, VA 23117 \\ Russell R. Irwin Phone: 703-894-5151}

\section{Accreditation Renewal Date: October 1, 1986}

This facility has been evaluated and deemed competent to process the radiation dosimeter listed below through employing Teledyne Automatic readers model 9100 and 9150, and Teledyne Manual readers model 8300 and 8310.

This facility is accredited to process the following dosimeter by virtue of actual demonstration of compliance with ANSI-N13.11-1983 through testing.

Teledyne TLD model BP3 for ANSI-N13.11 categories II, IV, V, VII. 


\section{CONSUMERS POWER COMPANY \\ PERSONNEL DOSIMETRY LABORATORY \\ 1945 Parnall Road, Jackson, MI 49201 \\ Theodore Allen \\ Phone: 517-788-2340}

Accreditation Renewall Date: October 1, 1986

This facility has been evaluated and deemed competent to process the radiation dosimeter listed below through employing a Teledyne Automatic reader model 9100.

This facility is accredited to process the following dosimeters by virtue of actual demonstration of compliance with ANSI-N13.11-1983 through testing.

Teledyne TLD model BG for ANSI-N13.11 categories II, IV, V, VII.

Teledyne TLD model BGN for ANSí-N13́.11 category VIII.

NVLAP LAB CODE 0523

\section{VIRGINIA ELECTRIC \& POWER COMPANY SURRY POWER STATION \\ P.O. Box 315, Surry, VA 23883 \\ Dean Densmore Phone: 804-357-3184}

Accreditation Renewal Date: January 1, 1987

This facility has been evaluated and deemed competent to process the radiation dosimeter listed below through employing Teledyne Automatic readers model 9100 and 9150, and Teledyne Manual reader model 8300.

This facility is accredited to process the following dosimeter by virtue of actual demonstration of compliance with ANSI-N13.11-1983 through testing.

Teledyne TLD model PB3 for ANSI-N13.11 categories II, IV, V, VII.

NVLAP LAB CODE 0524

\section{YANKEE ATOMIC ELECTRIC COMPANY \\ 1671 Worcester Road, Framingham, MA 01701 \\ Stephen T. Bard Phone: 617-872-8100}

Accreditation Renewal Date: October 1, 1986

This facility has been evaluated and deemed competent to process the radiation dosimeter listed below through employing a Harshaw Automatic reader model 2271.

This facility is accredited to process the following dosimeter by virtue of actual demonstration of compliance with ANSI-N13.11-1983 through testing.

Harshaw TLD model BGN for ANSI-N13.11 categories I, II, III, IV, V, VI, VII, and VIII.

NVLAP LAB CODE 0526

\section{KANSAS GAS AND ELECTRIC COMPANY WOLF CREEK GENERATING STATION \\ P.O. Box 309, Burlington, KS 66839 \\ Mike Nichols Phone: 316-364-8831}

Accreditation Renewal Date: January 1, 1987

This facility has been evaluated and deemed competent to process the radiation dosimeter listed below through employing a Panasonic Automatic reader model UD710A and manual reader 702E.

This facility is accredited to process the following dosimeter by virtue of actual demonstration of compliance with ANSI-N13.11-1983 through testing.

Panasonic TLD model UD802 for ANSI-N13.11 categories II, IV, V, VII, VIII. 
Index 1. Laboratory Name and NVLAP Lab Code Number

A \& $\mathrm{H} /$ Flood Engineering

Aguirre Engineers, Inc.

IL $\quad 0183$

American Carpet Laboratories, Inc.

$\mathrm{CO} \quad 0135$

American Testing

Apache Building Products Company

GA $\quad 0139$

Armstrong World Industries

Arnold Greene Testing Laboratories

PA $\quad 0146$

Atlantic Testing Labs, Ltd.

NJ $\quad 0218$

Baltimore Gas \& Electric Company

PA 0228

Bigelow-Sanford, Inc.

Bigelow-Sanford, Inc.

Butler Manufacturing Company

Central Ready-Mixed Concrete

CertainTeed Corporation

Certified Testing Laboratories, Inc.

MA 0225

Chemray Coatings Corp.

Chisholm Trail Testing and

NY $\quad 0177$

Commercial Testing Company

Conrock Co. Testing Laboratory

MD 0501

Construction Materials

GA 0156

Construction Technology Laboratories

$\mathrm{SC}$

0178

MO 0102

WI $\quad 0189$

PA 0101

Consumers Power Company

Contractors Supply Corporation

GA $\quad 0108$

Coronet Carpets

Custom Coating, Inc.

C. H. Masland and Sons

Dow Chemical USA, Foam Product

Dynatech R/D Company

$\mathrm{D} / \mathrm{L}$ Laboratories

E \& B Carpet Mills

NJ 0254

TX 0160

GA $\quad 0120$

$\mathrm{CA} \quad 0203$

$\mathrm{CO} \quad 0215$

Eberline Services Division

Factory Mutual Research Corp.

Galaxy Carpet Mills, Inc.

Garco Testing Laboratories

IL 0137

MI 0522

WV 0136

GA $\quad 0190$

GA 0243

PA 0187

$\mathrm{OH} \quad 0103$

MA 0113

NY $\quad 0252$

GA 0149

NM $\quad 0515$

MA 0115

GA $\quad 0163$

UT 0195

Genstar Stone Products Company

Geoscience Ltd.

Gifford-Hill \& Company, Inc.

Gold Bond Building Products

GPU Nuclear Corporation

Gulf Coast Testing Laboratory, Inc.

MD 0141

CA 0142

TX 0253

NY 0229

PA 0510

Hardwood Plywood

Harris Energy \&

TX 0208

VA 0151

NC $\quad 0517$

Hollytex Carpet Mills

OK $\quad 0247$

Houston Lighting \& Power Company

TX

0519

Hufcor Acoustical Laboratory

WI

0239

INTEST Laboratories, Inc.

MN

0119

Independent Textile Testing

GA

0166

Insta-Foam Products, Inc.

IL

NY 0511

James A. Fitzpatrick Nuclear

FL

0111

Jim Walter Research Corp.

TX

0143

Kansas Gas \& Electric Company

KS

0526 
NAHB Research Foundation, Inc.

MD $\quad 0104$

Naval Medical Command

Naval Research Laboratory

MD

Northwest Testing

DC $\quad 0509$

NY Power Authority, Indian Point

OR $\quad 0244$

OMNİ Environmental Services, Inc.

NY $\quad 0508$

Owens-Corning Fiberglas Corp

Owens-Corning Fiberglas Corp.

OR $\quad 0240$

Owens-Corning Fiberglas Corp.

KS $\quad 0126$

OH 0109

Owens-Corning Fiberglas Corp.

CA 0124

Owens-Corning Fiberglas Corp.

GA 0125

Owens-Corning Fiberglas Corp.

NJ 0127

Owens-Corning Fiberglas Corp.

NY $\quad 0128$

Owens-Corning Fiberglas Corp.

OH 0129

Pacific Inspection and

TX 0130

PFS Corporation

Pittsburgh Testing Laboratory

Pittsburgh Testing Laboratory

Radiation Detection Company

WA $\quad 0235$

WI 0223

Ritchie Laboratories

Riverbank Acoustical

PA 0201

NY 0237

$\mathrm{CA} \quad 0512$

KS $\quad 0232$

R. F. Geisser \& Associates

IL $\quad 0227$

R. S. Landauer Jr. \& Company

RI $\quad 0245$

R. W. Sidley, Inc.

IL 0518

Salem Carpet Laboratory

Shaw Industries, Inc., QC Lab

$\mathrm{OH} \quad 0206$

Smith-Emery Company

GA $\quad 0221$

Southern California Edison

GA 0193

Southwest Research Institute

CA 0192

CA 0506

Sparrell Engineering

STS Consultants, Ltd.

STS Consultants, Ltd.

STS Consultants, Ltd.

TX 0114

$\mathrm{ME} \quad 0121$

NC $\quad 0173$

State of CA, Dept. of Consumer Affairs

IL 0191

VA 0233

Stove Testing Lab

$\mathrm{CA} \quad 0251$

Stratton Laboratories

OR $\quad 0246$

Technical Micronics Control Inc.

Terralab Engineers

GA $\quad 0220$

Texas Testing Laboratories, Inc.

AL $\quad 0122$

UT 0199

The Arundel Corporation

The H. C. Nutting Company

MD

The Upjohn Company

The Walt Keeler Company, Inc.

Twin City Testing and Engineering Corp.

Underwriters Laboratories Inc.

Underwriters Laboratories Inc.

Underwriters Laboratories Inc.

United States Gypsum Company

United States Testing Company, Inc.

MN 0188

IL $\quad 0116$

$\begin{array}{ll}\text { CA } & 0117\end{array}$

NY 0255

IL $\quad 0216$

NJ $\quad 0105$

United States Testing Company, Inc.

United States Testing Company, Inc.

U.S. EPA, Nuclear Radiation

Virginia Concrete Laboratory

Virginia Electric \& Power Company

Virginia Electric \& Power Company

CA $\quad 0106$

OK $\quad 0107$

NV $\quad 0507$

VA $\quad 0230$

VA $\quad 0520$

VA $\quad 0523$ 
Warnock Hersey

West Virginia Dept of Highways

Western Electro-Acoustic

Western States Testing Div. of U.S. Testing

Wiss, Janney, Elstner and

World Carpets

W. R. Grace \& Company

W. R. Grace \& Company

Yankee Atomic Electric Company
WI

WV

CA

CA

IL

GA

MA

MA

MA
0249

0205

0256

0241

0226

0197

0176

0250

0524 
Acoustics LAP

0111

0119

0109

0227

0228

0229

0239

0256

0105

0106

0108

0114

0115

0116

0120

0139

0149

0151

0156

0160

0163

0166

0178

0187

0190

0193

0197

0220

0221

0243

0247

0255

0154

0131

0133

0135

0136

0137

0141

0143

0146

0173

0176

0177

0183

0188

0189

0191

0192

0195

0196

Jim Walter Reseach Corp.

INTEST Laboratories, Inc.

FL

Owens-Corning Fiberglas Corp.

Riverbank Acoustical

Armstrong World Industries

Gold Bond Building Products

Hufcor Acoustical Laboratory

Western Electro-Acoustic

\section{Carpet LAP}

United States Testing Company, Inc.

United States Testing Company, Inc.

Certified Testing Laboratories, Inc.

Southwest Research Institute

Factory Mutual Research Corp.

Underwriters Laboratories Inc.

Commercial Testing Company

American Carpet Laboratories, Inc.

E \& B Carpet Mills

Hardwood Plywood

Bigelow-Sanford, Inc.

Chisholm Trail Testing and

Galaxy Carpet Mills, Inc.

Independent Textile Testing

Bigelow-Sanford, Inc.

C. H. Masland and Sons

Coronet Carpets

Shaw Industries, Inc., QC Lab

World Carpets

Stratton Laboratories

Salem Carpet Laboratory

Custom Coating, Inc.

Hollytex Carpet Mills

Underwriters Laboratories Inc.

NJ

CA

GA

TX

MA

IL

GA

GA

GA

VA

GA

TX

GA

GA

$\mathrm{SC}$

PA

GA

GA

GA

GA

GA

GA

OK

NY

Concrete LAP

The Arundel Corporation

The H. C. Nutting Company

MD

$\mathrm{OH}$

The Walt Keeler Company, Inc.

KS

Aguirre Engineers, Inc.

$\mathrm{CO}$

Contractors Supply Corporation

WV

Construction Technology Laboratories

IL

Genstar Stone Products Company

MD

Kelso Industries, Inc.

TX

American Testing

STS Consultants, Ltd.

PA

W. R. Grace \& Company

NC

Atlantic Testing Labs, Ltd.

MA

NY

A \& $\mathrm{H} /$ Flood Engineering

IL

Twin City Testing and Engineering

MN

Central Ready-Mixed Concrete

WI

STS Consultants, Ltd.

IL

Smith-Emery Company

Garco Testing Laboratories

CA

Texas Testing Laboratories, Inc.

UT

TX 
R. W. Sidley, Inc.

Gulf Coast Testing Laboratory, Inc. $\quad$ TX

0208

0215

Construction Materials

$\mathrm{CO}$

Virginia Concrete Laboratory

VA

Ritchie Laboratories

KS

STS Consultants, Ltd. VA

0233

0237

Pittsburgh Testing Laboratory

NY

Western States Testing Div. of U.S. Testing CA

Commercial Products LAP

D/L Laboratories

0254 Chemray Coatings Corp.

Baltimore Gas \& Electric Company

Mallinckrodt Diagnostics, Inc.

Naval Medical Command

Southern California Edison

0507

U.S. EPA, Nuclear Radiation

CA

NY Power Authority, Indian Point

NV

0508

Naval Research Laboratory

NY

0509

GPU Nuclear Corporation

DC

0510

James A. Fitzpatrick Nuclear

PA

0511

Radiation Detection Company

NY

0512

Eberline Services Division

$\mathrm{CA}$

0515

Harris Energy \&

0517

R. S. Landauer Jr. \& Company

0519 Houston Lighting \& Power Company

$\mathrm{NM}$

$\mathrm{NC}$

IL

Virginia Electric \& Power Company

TX

Consumers Power Company

VA

0522

Virginia Electric \& Power Company

MI

0523

Yankee Atomic Electric Company

VA

0524

Kansas Gas \& Electric Company

MA

$\mathrm{KS}$

Stove LAP

Underwriters Laboratories Inc.

IL

Underwriters Laboratories Inc.

CA

PFS Corporation

WI

Arnold Greene Testing Laboratories

MA

Pacific Inspection and

WA

OMNI Environmental Services, Inc.

OR

Northwest Testing

OR

R.F. Geisser \& Associates

RI

Stove Testing Lab

OR

Warnock Hersey

WI

Thermal Insulation LAP

CertainTeed Corporation

PA

Butler Manufacturing Company

MO

Dow Chemical USA, Foam Product

$\mathrm{OH}$

NAHB Research Foundation, Inc.

MD

United States Testing Company, Inc.

NJ

United States Testing Company, Inc.

$\mathrm{CA}$

United States Testing Company, Inc.

OK 
Commercial Testing Company

Manville Corporation

Owens-Corning Fiberglas Corp.

Owens-Corning Fiberglas Corp.

Owens-Corning Fiberglas Corp

Owens-Corning Fiberglas Corp.

Owens-Corning Fiberglas Corp.

NY

Owens-Corning Fiberglas Corp.

$\mathrm{OH}$

Owens-Corning Fiberglas Corp.

TX

Geoscience Ltd.

Hardwood Plywood

The Upjohn Company

Twin City Testing and

Terralab Engineers

Insta-Foam Products, Inc. 
Index 3. Accredited Laboratories by State and NVLAP Code Number

AL Technical Micronics Contol Inc.

CA United States Testing Company, Inc.

0106

CA Underwriters Laboratories Inc.

0117

CA Owens-Corning Fiberglas Corp.

0124

CA Geoscience Ltd.

0142

CA Smith-Emery Company

0192

CA Conrock Co. Testing Laboratory

0203

CA Western States Testing Div. of U.S. Testing

CA Western Electro-Acoustic

CA Southern California Edison

CA Radiation Detection Company

0512

CA State of CA, Dept. of Consumer Affairs 0251

CO Manville Corporation

0123

$\mathrm{CO}$ Aguirre Engineers, Inc.

0135

$\mathrm{CO}$ Construction Materials

CT The Upjohn Company

0175

DC Naval Research Laboratory

FL Jim Walter Research Corp.

GA Certified Testing Laboratories, Inc.

GA Commercial Testing Company

GA Owens-Corning Fiberglas Corp.

GA American Carpet Laboratories, Inc.

GA E \& B Carpet Mills

GA Bigelow-Sanford, Inc.

GA Galaxy Carpet Mills, Inc.

GA Independent Textile Testing

GA Coronet Carpets

GA Shaw Industries, Inc., QC Lab 0193

GA World Carpets

GA Stratton Laboratories

GA Salem Carpet Laboratory

GA Custom Coating, Inc.

IL Underwriters Laboratories Inc.

$\begin{array}{ll}\text { IL } & 0137\end{array}$

IL A \& H/Flood Engineering $\quad 0183$

IL STS Consultants, Ltd.

IL Insta-Foam Products, Inc. $\quad 0210$

IL United States Gypsum Company 0216

IL Wiss, Janney, Elstner and 0226

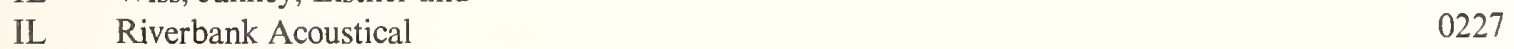

IL R. S. Landauer Jr. \& Company 0518

IN Knauf Fiber Glass Research $\quad 0248$

KS Owens-Corning Fiberglas Corp $\quad 0126$

KS The Walt Keeler Company, Inc. 0133

KS Ritchie Laboratories $\quad 0232$

KS Kansas Gas \& Electric Company 0526

MA Dynatech R/D Company 0113

MA Factory Mutual Research Corp. $\quad 0115$

MA W. R. Grace \& Company $\quad 0176$

MA Arnold Greene Testing Laboratories 0225

MA W. R. Grace \& Company $\quad 0250$

MA Yankee Atomic Electric Company 0524

MD The Arundel Corporation $\quad 0154$

MD NAHB Research Foundation, Inc. 0104

MD Genstar Stone Products Company 0141

MD Baltimore Gas \& Electric Company $\quad 0501$ 


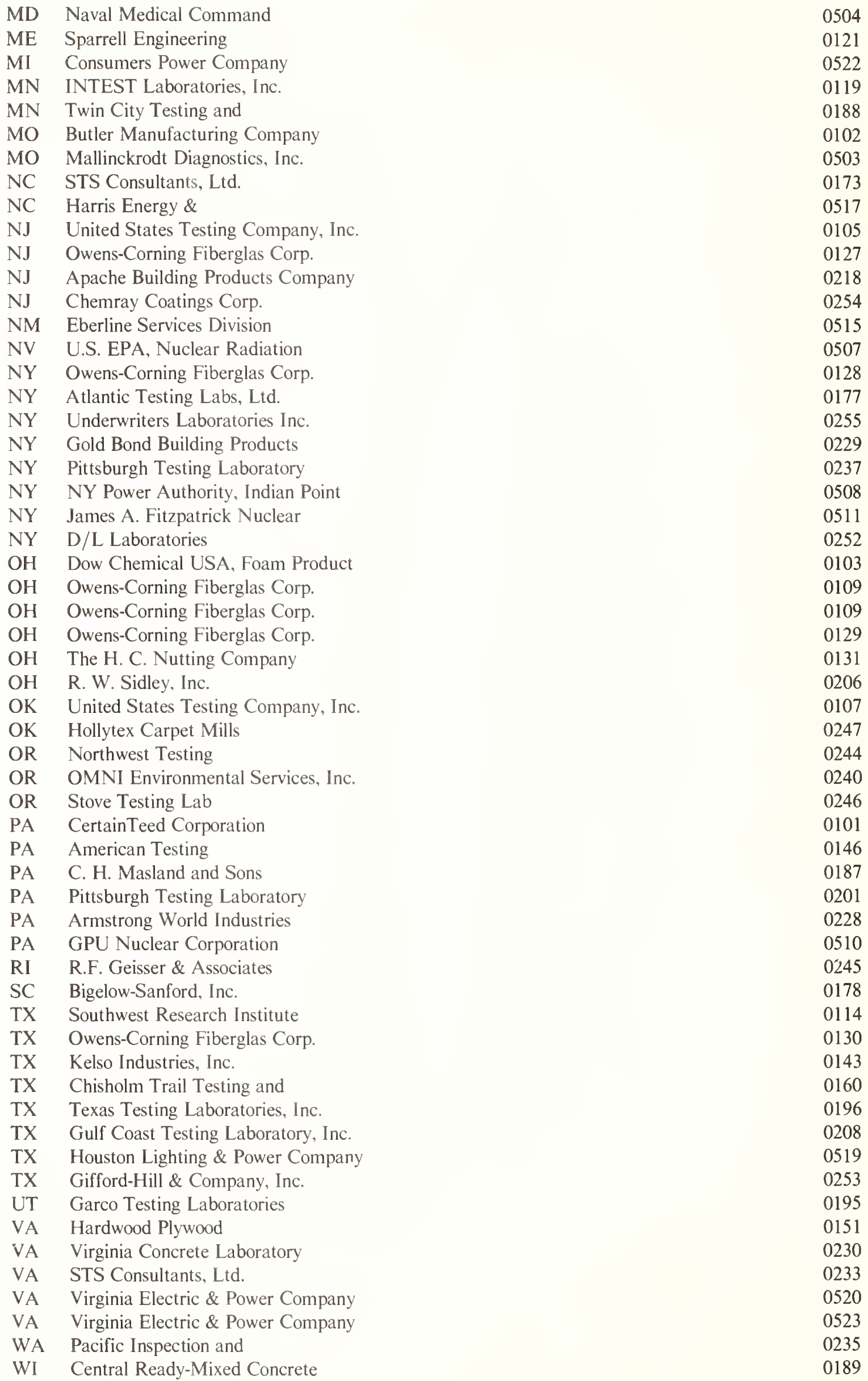


WI Hufcor Acoustical Laboratory

WI Warnock Hersey

0249

WV Contractors Supply Corporation

0136

WV West Virginia Dept of Highways

0205 


\section{Index 4. Test Methods Available Under Each LAP and NVLAP Code Numbers of Laboratories Accredited for those Test Methods}

This index provides a cross reference of accredited laboratories with test methods under each LAP. Laboratory code numbers under each test method refer to the laboratories for which the name, address, primary contact, phone number, and list of accredited test methods are identified in the Directory.

INSULATION LAP-CORROSIVENESS TEST METHODS

\begin{tabular}{llc}
$01 / \mathrm{C} 01$ & ASTM C739 & 0109,0116 \\
$01 / \mathrm{C} 02$ & HH-I-515 & $0101,0106,0107,0109,0115,0116,0120$ \\
01/C03 & California Energy Commission tests for insulating materials: Corrosiveness \\
& \multicolumn{2}{c}{0101,0109}
\end{tabular}

\section{INSULATION LAP-DIMENSION, STABILITY, AND DENSITY TEST METHODS}

$\begin{array}{lll}01 / \text { D01 } & \text { ASTM C136 } & 0101,0109,0116 \\ 01 / \text { D02 } & \text { ASTM C167 } & 0101,0104,0109,0116,0123,0124,0126,0127,0128,0129,0130,0248 \\ 01 / \text { D03 } & \text { ASTM C209 } & 0109,0111,0116,0123 \\ 01 / \text { D04 } & \text { ASTM C209 } & 0109,0111,0116,0123 \\ 01 / \text { D05 } & \text { ASTM C209 } & 0109,0111,0116,0123 \\ 01 / \text { D06 } & \text { ASTM C209 } & 0109,0111,0116,0123 \\ 01 / \text { D07 } & \text { ASTM C272 } & 0109,0111 \\ 01 / \text { D08 } & \text { ASTM C302 } & 0101,0109,0116,0123,0142,0248 \\ 01 / \text { D09 } & \text { ASTM C303 } & 0101,0109,0111,0116,0123,0124,0126,0127,0129,0130,0248,0250 \\ 01 / \text { D11 } & \text { ASTM C356 } & 0109,0123,0248 \\ 01 / \text { D12 } & \text { ASTM C411 } & 0109,0123,0248 \\ 01 / \text { D13 } & \text { ASTM C519 } & 0101,0104,0109,0116,0117,0123,0248 \\ 01 / \text { D14 } & \text { ASTM C520 } & 0116,0250 \\ 01 / \text { D15 } & \text { ASTM D756 } & 0109,0210 \\ 01 / \text { D16 } & \text { ASTM D756 } & 0109,0210 \\ 01 / \text { D17 } & \text { ASTM D756 } & 0109,0210 \\ 01 / \text { D18 } & \text { ASTM D1622 } & 0103,0107,0109,0116,0210,0218\end{array}$

\section{INSULATION LAP-VAPOR BARRIER PROPERTIES TEST METHODS}

$\begin{array}{lll}01 / \text { D19 } & \text { ASTM D2126 } & 0109 \\ 01 / \text { D20 } & \text { ASTM D2126 } & 0109,0111,0210 \\ 01 / D 21 & \text { ASTM D2126 } & 0103,0106,0109,0111,0175,0199,0218 \\ 01 / \text { D22 } & \text { ASTM D2126 } & 0109,0210 \\ 01 / D 23 & \text { ASTM D2842 } & 0103,0109,0210 \\ 01 / D 24 & \text { ASTM C739 } & 0109,0116 \\ 01 / D 25 & \text { HH-I-515 } & 0101,0107,0109,0115,0116,0120,0122,0199 \\ 01 / D 26 & \text { HH-I-515 } & 0101,0107,0109,0115,0116,0117,0120,0122 \\ 01 / \text { D27 } & \text { ASTM D2126 } & 0103,0106,0109,0210,0218 \\ 01 / D 28 & \text { ASTM D2126 } & 0106,0109,0175,0199,0210 \\ 01 / D 29 & \text { California Energy Commission tests for insulating materials: } & 0109\end{array}$

\section{INSULATION LAP-FIRE PROPERTIES TEST METHODS}

$\begin{array}{lll}\text { 01/F01 } & \text { TAPPI T461 } & 0101,0109,0123 \\ \text { 01/F02 } & \text { ASTM E84 } & 0105,0106,0109,0111,0115,0116,0117,0123,0151,0199 \\ \text { 01/F05 } & \text { ASTM E136 } & 0101,0106,0109,0123,0142 \\ \text { 01/F06 } & \text { ASTM C739 } & 0116 \\ \text { 01/F07 } & \text { HH-I-515 } & 0101,0105,0106,0109,0115,0116,0117,0120,0122,0151,0199,0251 \\ \text { 01/F08 } & \text { HH-I-515 } & 0101,0107,0109,0115,0116,0117,0120,0122,0251\end{array}$




\section{INSULATION LAP-STRENGTH PROPERTIES TEST METHODS}

\begin{tabular}{lll}
$01 / \mathrm{S} 01$ & ASTM C165 & $0101,0109,0123,0248$ \\
$01 / \mathrm{S} 02$ & ASTM C203 & $0103,0109,0111,0116,0123$ \\
$01 / \mathrm{S} 03$ & ASTM C209 & $0109,0111,0116,0123$ \\
$01 / \mathrm{S} 04$ & ASTM C209 & $0109,0111,0116,0123$ \\
$01 / \mathrm{S} 05$ & ASTM C209 & $0109,0111,0116,0123$ \\
$01 / \mathrm{S} 06$ & ASTM C209 & $0109,0111,0116,0123$ \\
$01 / \mathrm{S} 07$ & ASTM C273 & 0103,0109, \\
$01 / \mathrm{S} 08$ & ASTM C446 & $0101,0109,0116,0123$ \\
$01 / \mathrm{S} 09$ & ASTM D781 & $0101,0109,0123$ \\
$01 / \mathrm{S} 10$ & ASTM D828 & $0101,0109,0123$ \\
$01 / \mathrm{S} 11$ & ASTM D1621 & $0103,0109,0111,0116,0199,0210,0218$ \\
$01 / \mathrm{S} 12$ & California Energy Commission tests for insulating materials: \\
& \multicolumn{2}{c}{0101} \\
$01 / \mathrm{S} 13$ & California Energy Commission tests for insulating materials: \\
$01 / \mathrm{S} 14$ & California Energy Commission tests for insulating materials:
\end{tabular}

\section{INSULATION LAP-THERMAL PROPERTIES TEST METHODS}

$\begin{array}{lll}01 / \text { T01 } & \text { ASTM C177 } & 0101,0109,0111,0113,0121,0123,0142,0248 \\ 01 / \text { T04 } & \text { ASTM C236 } & 0101,0102,0109,0111,0113,0121,0123,0142,0188,0226,0250 \\ \text { 01/T05 } & \text { ASTM C335 } & 0101,0109,0111,0113,0123,0210,0248 \\ 01 / \text { T06 } & \text { ASTM C518 } & 0101,0102,0103,0104,0105,0109,0111,0113,0116,0120,0121,0122,0123, \\ & & 0124,0125,0126,0127,0128,0129,0130,0175,0199,0210,0216,0218,0248, \\ & & 0250 \\ 01 / \text { T09 } & \text { ASTM C653 } & 0101,0104,0109,0116,0123,0248 \\ 01 / \text { T10 } & \text { ASTM C687 } & 0101,0104,0109,0116,0123,0248\end{array}$

\section{INSULATION LAP-OTHER TEST METHODS}

01/V02 TAPPI T419 0109, 0116

01/V03 ASTM D2020 0109, 0116

$01 / \mathrm{V} 04$ ASTM E96

$0101,0103,0106,0107,0109,0111,0123,0210$

$01 / \mathrm{V} 05$

HH-I-515

01/V06 HH-I-515

$0107,0109,0116$

$0107,0116,0122$

\section{CONCRETE LAP-FIELD TEST METHODS}

$\begin{array}{ll}\text { 02/M01 } & \text { ASTM C31 } \\ \text { 02/M03 } & \text { ASTM C172 } \\ \text { 02/P01 } & \text { ASTM C143 } \\ \text { 02/W01 } & \text { ASTM C138 } \\ \text { 02/A01 } & \text { ASTM C231 }\end{array}$

\section{CONCRETE LAP-FIELD PLUS LABORATORY TEST METHODS}

Field Test Methods listed above plus:
02/S01 ASTM C39 0131, 0135, 0136, 0137, 0141, 0143, 0146, 0154, 0173, 0176, 0177, 0183, 0188, 0189, 0191, 0192, 0195, 0196, 0201, 0203, 0205, 0206, 0208, 0215, 0230, 0231, 0232, 0233, 0237, 0241, 0253

\section{CONCRETE LAP-OPTIONAL TEST METHOD}

02/A02 ASTM C173 0131, 0133, 0135, 0137, 0141, 0143, 0146, 0154, 0173, 0176, 0177, 0183, 0188, 0191, 0192, 0195, 0196, 0201, 0203, 0205, 0206, 0208, 0215, 0230, 0232, 0237, 0241,0253 


\section{CARPET LAP}

\begin{tabular}{|c|c|c|}
\hline 03/C01 & AATCC $16 \mathrm{E}$ & $\begin{array}{l}0105,0106,0108,0120,0139,0149,0156,0160,0163,0166,0178,0187,0190 \text {, } \\
0193,0197,0221\end{array}$ \\
\hline 03/C02 & AATCC 8 & $\begin{array}{l}0105,0108,0120,0139,0149,0156,0160,0163,0166,0178,0187,0190,0193 \text {, } \\
0197,0221,0247\end{array}$ \\
\hline 03/D01 & ASTM D418 & $\begin{array}{l}0105,0106,0108,0120,0139,0149,0156,0160,0163,0166,0178,0187,0190 \text {, } \\
0193,0197,0221\end{array}$ \\
\hline 03/D02 & DDD-C-95A & $\begin{array}{l}0105,0108,0120,0139,0149,0156,0160,0163,0166,0178,0187,0190,0193 \text {, } \\
0197,0221\end{array}$ \\
\hline 03/S01 & ASTM D1335 & $\begin{array}{l}0105,0108,0120,0139,0149,0156,0160,0163,0166,0178,0187,0190,0193 \text {, } \\
0197,0220,0221,0247\end{array}$ \\
\hline 03/E01 & AATCC $134 / C R I$ & $\begin{array}{l}102 \\
0108,0166,0178\end{array}$ \\
\hline 03/F01 & ASTM E84 & $0105,0106,0114,0115,0116,0120,0151$ \\
\hline 03/F02 & UL 992 & 0114,0116 \\
\hline 03/F03 & DoC FF1-70 & $\begin{array}{l}0105,0106,0108,0114,0116,0120,0139,0149,0156,0160,0163,0166,0178, \\
0187,0190,0193,0197,0220,0221,0243,0247,0255\end{array}$ \\
\hline 03/F04 & ASTM E648 & $0105,0106,0108,0114,0115,0116,0120,0151,0166,0178,0220,0221,0255$ \\
\hline 03/B01 & UM 44C Addendur & 0156,0178 \\
\hline 03/B02 & UM 44C Addenda & $\begin{array}{l}2 \text { and } 3 \\
0105,0108,0120,0139,0163,0166\end{array}$ \\
\hline
\end{tabular}

STOVE LAP

Physical/Fire Group

Physical/Fire Group

Physical/Fire Group and Mobile Home Group

Physical/Fire Group and Mobile Home Group

Physical/Fire Group and Electrical Group

Physical/Fire Group and Electrical Group

Physical/Fire Group and Mobile Home Group and Electrical Group

Physical/Fire Group and Mobile Home Group and Electrical Group
08/P01
08/P02
08/P03
08/P04
08/P05
08/P06
08/P07
08/P08
08/P09
08/P10
08/P11
08/P12

UL 737, UL 1482

CSA B336.2

UL 737, UL 1482

CSA B336.2

UL 737, UL 1482

CSA C 22.2 Nos. 103 and 113

UL 737, UL 1482

$0116,0117,0223,0225,0235,0240,0244,0245,0246,0249$

CSA B336.2, CSA C 22.2 No. 103, and CSA C 22.2 No. 113

\section{ACOUSTICS LAP-PRECISION TEST METHODS}

$\begin{array}{ll}\text { ASTM C367-78 } & 0109 \\ \text { ASTM C384-77 } & 0109,0111,0119,0123 \\ \text { ASTM C423-81 } & 0109,0111,0119,0123,0227,0228,0229 \\ \text { ASTM C522-80 } & 0109,0123 \\ \text { ASTM C523-68 }(81) & 0109,0227,0229 \\ & 0109,0111,0119,0123,0227,0229,0239 \\ \text { ASTM E90-82 } & 010 \\ \text { ASTM E492-82 } & 0227,0228 \\ \text { ASTM E596-78 } & \\ \text { ASTM E756-82 } & \\ \text { ANSI S1.31-80 } & 0109,0227 \\ \text { ANSI S1.31-80 } & 0119 \\ \text { ANSI S1.31-80 } & \end{array}$




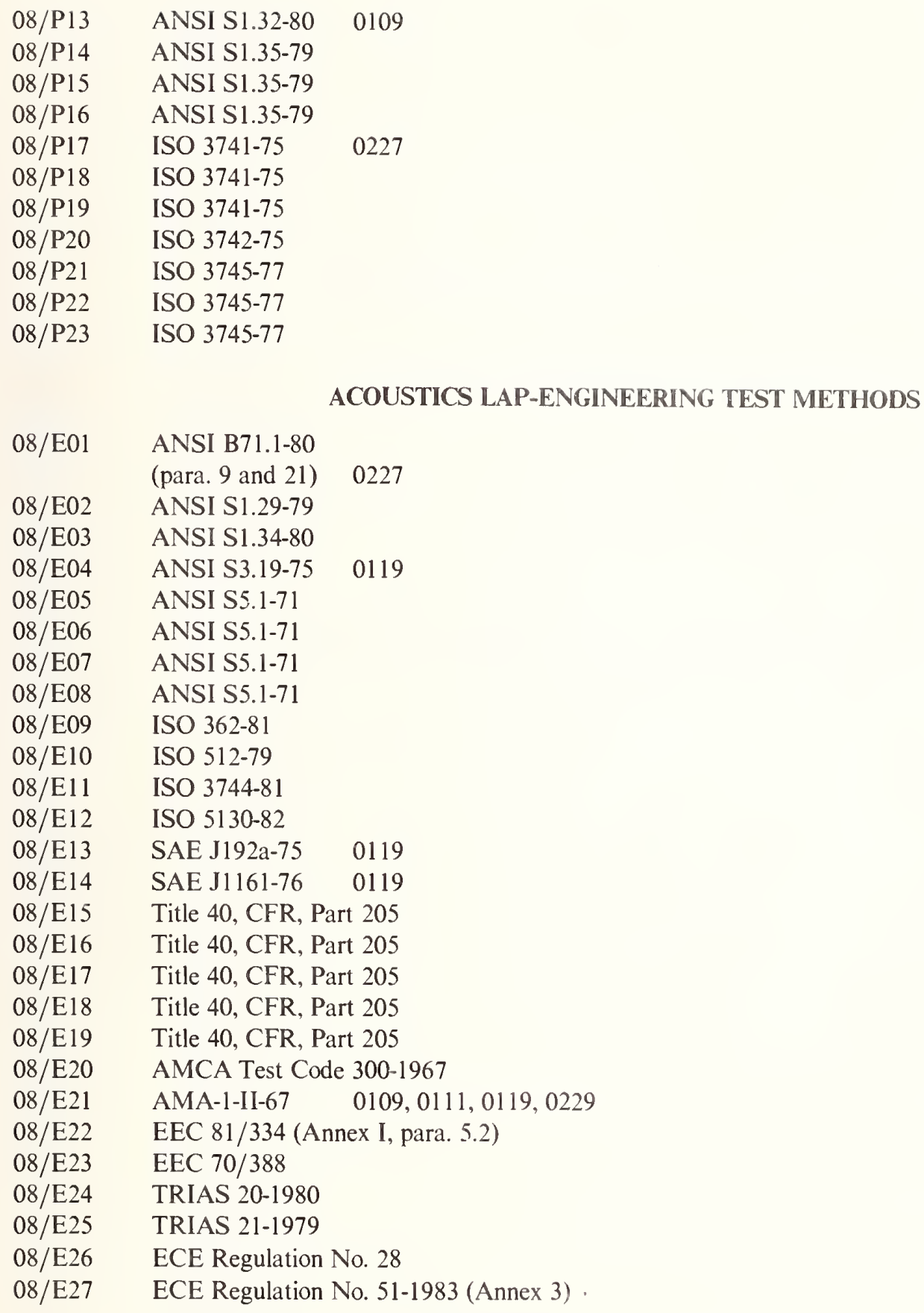

ACOUSTICS LAP-ENGINEERING TEST METHODS

08/E01

08/E02

08/E03

08/E04

08/E05

08/E06

08/E07

08/E08

08/E09

$08 / \mathrm{E} 10$

08/E11

08/E12

$08 / \mathrm{E} 13$

$08 /$ E14

08/E15

08/E16

08/E17

08/E18

08/E19

08/E20

08/E21

08/E22

08/E23

08/E24

08/E25

08/E26

08/E27

ANSI B71.1-80

(para. 9 and 21)

0227

ANSI S1.29-79

ANSI S1.34-80

ANSI S3.19-75

0119

ANSI S5.1-71

ANSI S5.1-71

ANSI S5.1-71

ANSI S5.1-71

ISO $362-81$

ISO $512-79$

ISO $3744-81$

ISO $5130-82$

SAE J192a-75 0119

SAE J1161-76 0119

Title 40, CFR, Part 205

Title 40, CFR, Part 205

Title 40, CFR, Part 205

Title 40, CFR, Part 205

Title 40, CFR, Part 205

AMCA Test Code 300-1967

AMA-1-II-67 0109, 0111, 0119, 0229

EEC 81/334 (Annex I, para. 5.2)

EEC 70/388

TRIAS 20-1980

TRIAS 21-1979

ECE Regulation No. 28

ECE Regulation No. 51-1983 (Annex 3) .

COMMERCIAL PRODUCTS LAP

See the entries in the Directory for NVLAP Lab Code Numbers 0252 and 0254 for accredited test methods.

PAINTS AND RELATED COATINGS AND MATERIALS

Measurements of Intrinsic Physical Properties

$\begin{array}{ll}\text { 09/A01 } & \text { ASTM D56 } \\ \text { 09/A02 } & \text { ASTM D93 } \\ \text { 09/A03 } & \text { ASTM D153 } \\ \text { 09/A04 } & \text { ASTM D185 } \\ \text { 09/A05 } & \text { ASTM D281 } \\ \text { 09/A06 } & \text { ASTM D387 } \\ \text { 09/A07 } & \text { ASTM D523 } \\ \text { 09/A08 } & \text { ASTM D562 } \\ \text { 09/A09 } & \text { ASTM D1005 }\end{array}$




$\begin{array}{ll}\text { 09/A10 } & \text { ASTM D1186 } \\ \text { 09/A11 } & \text { ASTM D1200 } \\ \text { 09/A12 } & \text { ASTM D1210 } \\ \text { 09/A13 } & \text { ASTM D1212 } \\ \text { 09/A14 } & \text { ASTM D1296 } \\ \text { 09/A15 } & \text { ASTM D1310 } \\ \text { 09/A16 } & \text { ASTM D1400 } \\ \text { 09/A17 } & \text { ASTM D1475 } \\ \text { 09/A18 } & \text { ASTM D1544 } \\ \text { 09/A19 } & \text { ASTM D1729 } \\ \text { 09/A20 } & \text { ASTM D2244 } \\ \text { 09/A21 } & \text { ASTM D3278 } \\ \text { 09/A22 } & \text { ASTM D3363 } \\ \text { 09/A23 } & \text { ASTM D3793 } \\ \text { 09/A24 } & \text { ASTM D4061 } \\ \text { 09/A25 } & \text { ASTM D4212-82 } \\ \text { 09/A26 } & \text { ASTM E97 } \\ \text { 09/A27 } & \text { ASTM E308 } \\ \text { 09/A28 } & \text { ASTM E313 } \\ \text { 09/A29 } & \text { ASTM E430 }\end{array}$

Measurements of Performance and Performance Change

$\begin{array}{ll}\text { 09/B01 } & \text { ASTM D279 } \\ \text { 09/B02 } & \text { ASTM D332 } \\ \text { 09/B03 } & \text { ASTM D344 } \\ \text { 09/B04 } & \text { ASTM D610 } \\ \text { 09/B05 } & \text { ASTM D659 } \\ \text { 09/B06 } & \text { ASTM D660 } \\ \text { 09/B07 } & \text { ASTM D661 } \\ \text { 09/B08 } & \text { ASTM D662 } \\ \text { 09/B09 } & \text { ASTM D711 } \\ \text { 09/B10 } & \text { ASTM D714 } \\ \text { 09/B11 } & \text { ASTM D772 } \\ \text { 09/B12 } & \text { ASTM D821 } \\ \text { 09/B13 } & \text { ASTM D868 } \\ \text { 09/B14 } & \text { ASTM D869 } \\ \text { 09/B15 } & \text { ASTM D880 } \\ \text { 09/B16 } & \text { ASTM D913 } \\ \text { 09/B17 } & \text { ASTM D968 } \\ \text { 09/B18 } & \text { ASTM D969 } \\ \text { 09/B19 } & \text { ASTM D1308 } \\ \text { 09/B20 } & \text { ASTM D1309 } \\ \text { 09/B21 } & \text { ASTM D1360 } \\ \text { 09/B22 } & \text { ASTM D1543 } \\ \text { 09/B23 } & \text { ASTM D1640 } \\ \text { 09/B24 } & \text { ASTM D1737 } \\ \text { 09/B25 } & \text { ASTM D2197 } \\ \text { 09/B26 } & \text { ASTM D2243 } \\ \text { 09/B27 } & \text { ASTM D2248 } \\ \text { 09/B28 } & \text { ASTM D2366 } \\ \text { 09/B29 } & \text { ASTM D2486 } \\ \text { 09/B30 } & \text { ASTM D2801 } \\ \text { 09/B31 } & \text { ASTM D2805 } \\ \text { 09/B32 } & \text { ASTM D3273 } \\ \text { 09/B33 } & \text { ASTM D3274 } \\ \text { 09/B34 } & \text { ASTM D3450 } \\ \text { 09/B35 } & \text { ASTM D3456 } \\ \text { 09/B36 } & \text { ASTM D3623 } \\ & \end{array}$




$\begin{array}{ll}\text { 09/B37 } & \text { ASTM D4060 } \\ \text { 09/B38 } & \text { ASTM D4062 } \\ \text { 09/B39 } & \text { ASTM D4213 } \\ \text { 09/B40 } & \text { ASTM D4214 } \\ \text { 09/B41 } & \text { Fed. Std. 141 } \\ & \text { Method 4494 } \\ \text { 09/B42 } & \begin{array}{l}\text { Fed. Std. 141 } \\ \text { Method 4061 }\end{array}\end{array}$

09/C01 ASTM D34

09/C02 ASTM D95

09/C03 ASTM D521

09/C04 ASTM D563

09/C05 ASTM D611

09/C06 ASTM D1078

09/C07 ASTM D1133

09/C08 ASTM D1208

09/C09 ASTM D1259

09/C10 ASTM D1306

09/C11 ASTM D1353

09/C12 ASTM D1364

09/C13 ASTM D1394

09/C14 ASTM D1397

09/C15 ASTM D 1398

09/C16 ASTM D1399

09/C17 ASTM D1467

09/C18 ASTM D1469

09/C19 ASTM D1541

09/C20 ASTM D 1613

09/C21 ASTM D1639

09/C22 ASTM D1644

09/C23 ASTM D1652

09/C24 ASTM D2075

09/C25 ASTM D2076

09/C26 ASTM D2369

09/C27 ASTM D2371

09/C28 ASTM D2697

09/C29 ASTM D2698

09/C30 ASTM D2832

09/C31 ASTM D3009

09/C32 ASTM D3271

09/C33 ASTM D3272

09/C34 ASTM D3335

09/C35 ASTM D3624

09/C36 ASTM D3718

09/C37 ASTM D3723

09/C38 ASTM D3792

09/C39 ASTM D3960

09/C40 ASTM D4017

09/D01 ASTM B117

09/D02 ASTM D609

09/D03 ASTM D822

09/D04 ASTM D823

09/D05 ASTM D1106

09/D06 ASTM D1014

Measurement of Chemical Properties and Compositions 


$\begin{array}{ll}\text { 09/D07 } & \text { ASTM D1654 } \\ \text { 09/D08 } & \text { ASTM D1730 } \\ \text { 09/D09 } & \text { ASTM D1734 } \\ \text { 09/D10 } & \text { ASTM D2247 } \\ \text { 09/D11 } & \text { ASTM D2372 } \\ \text { 09/D12 } & \text { ASTM D3361 } \\ \text { 09/D13 } & \text { ASTM D3924 } \\ \text { 09/D14 } & \text { ASTM G23 } \\ \text { 09/D15 } & \text { ASTM G26 } \\ \text { 09/D16 } & \text { ASTM G53 }\end{array}$

PAPER AND RELATED PRODUCTS

Paper and Paperboard

$\begin{array}{ll}\text { 09/E01 } & \text { TAPPI T208-OS } \\ \text { 09/E02 } & \text { TAPPI T402-OM } \\ & \text { ASTM D685 } \\ \text { 09/E03 } & \text { TAPPI T403-OS } \\ & \text { ASTM D774 } \\ \text { 09/E04 } & \text { TAPPI T404-OM } \\ & \text { ASTM D828 } \\ \text { 09/E05 } & \text { TAPPI T410-OM } \\ \text { 09/E06 } & \text { TAPPI T411-OM } \\ \text { 09/E07 } & \text { TAPPI T412-OM } \\ & \text { ASTM D644 } \\ \text { 09/E08 } & \text { TAPPI T414-OM } \\ & \text { ASTM D689 } \\ \text { 09/E09 } & \text { TAPPI T425-OM } \\ \text { 09/E10 } & \text { TAPPI T435-OM } \\ \text { 09/E11 } & \text { TAPPI T452-OM } \\ \text { 09/E12 } & \text { TAPPI T459-OM } \\ & \text { ASTM D2482 } \\ \text { 09/E13 } & \text { TAPPI T460-OM } \\ & \text { ASTM D726 } \\ \text { 09/E14 } & \text { TAPPI T480-OM } \\ \text { 09/E15 } & \text { TAPPI T480-OS } \\ \text { 09/E16 } & \text { TAPPI T489-OS } \\ \text { 09/E17 } & \text { TAPPI T494-OM } \\ \text { 09/E18 } & \text { TAPPI T511-OM } \\ & \text { ASTM D2176 } \\ \text { 09/E19 } & \text { TAPPI T538-PM } \\ \text { 09/E20 } & \text { TAPPI T809-OM } \\ \text { 09/E21 } & \text { TAPPI T818-OM } \\ & \text { ASTM D1164 }\end{array}$

Paper Specifications

$\begin{array}{ll}\text { 09/F01 } & \begin{array}{l}\text { ASTM D3208 } \\ \text { para. 11 } \\ \text { 09/F02 }\end{array} \\ & \begin{array}{l}\text { ASTM D3290 } \\ \text { para. 11.2 }\end{array} \\ & \\ \text { 09/G01 } & \begin{array}{l}\text { ASTM D3330, } \\ \text { D3330M }\end{array} \\ \text { 09/G02 } & \begin{array}{l}\text { ASTM D3652 } \\ \text { ASTM D3654, }\end{array} \\ \text { 09/G03 } & \begin{array}{l}\text { D3654M } \\ \text { ASTM D3662 }\end{array} \\ 09 / \text { G04 } & \end{array}$

Pressure Sensitive Tapes 


$\begin{array}{ll}\text { 09/G05 } & \text { ASTM D3759 } \\ \text { 09/G06 } & \text { ASTM D381 } 1 \\ 09 / \text { G07 } & \text { ASTM D3815 }\end{array}$

Packaging

$\begin{array}{ll}\text { 09/H01 } & \text { ASTM D642 } \\ \text { 09/H02 } & \text { ASTM D895 } \\ \text { 09/H03 } & \text { ASTM D1108 }\end{array}$

Federal Test Method Standard 101C for Preservation, Packaging, and Packaging Materials

09/H04 Method 4035

09/H05 Method 4047

09/H06 Method 5001

09/H07 Method 5005.1

09/H08 Method 5007.1

09/H09 Method 5008.1

09/H10 Method 5009.2

09/H11 Method 5011.1

09/H12 Method 5012

09/H13 Method 5013

09/H14 Method 5014

09/H15 Method 5015

09/H16 Method 5016.1

09/H17 Method 5017

09/H18 Method 5018

09/H19 Method 5019.1

09/H20 Method 5020.1

09/H21 Method 5023

09/H22 Method 5026

\section{MATTRESSES}

$\begin{array}{ll}\text { 09/K01 } & \text { 16 CFR Part 1632 } \\ & \text { Sec. 1632.4 } \\ \text { 09/K02 } & \text { MIL-R-0020092J(SH) } \\ & \text { Sec. 4.4 } \\ 09 / \text { K03 } & \text { MIL-M-18251F } \\ & \text { Sec. 4.5.1 } \\ 09 / \text { K04 } & \text { CCC- C-436D } \\ & \text { Sec. 4.4 } \\ 09 / \text { K05 } & \text { V-M-96H } \\ & \text { Sec. 4.4.1.1 } \\ & \text { \& Sec. 4.5 } \\ 09 / \text { K06 } & \text { AH\&MA/NABM }\end{array}$


ANSI N13.11-1983

I. Accidents, Low energy photons 0501, 0506, 0508, 0510, 0512, 0515, 0517, 0518, 0524

II. Accidents, High energy photons $0501,0504,0506,0508,0509,0510,0511,0512,0515,0517,0518,0520,0520,0522,0523$, 0524,0526

III. Protection, Low energy photons 0501, 0504, 0506, 0508, 0509, 0510, 0512, 0515, 0517, 0518, 0524

IV. Protection, High energy photons 0501, 0504, 0506, 0507, 0508, 0509, 0510, 0511, 0512, 0515, 0517, 0518, 0519, 0520, 0522, $0523,0524,0526$

V. Protection, Beta particles $0501,0504,0506,0508,0510,0512,0515,0517,0518,0520,0522,0523,0524,0526$

VI. Protection, Photon mixtures $0501,0504,0506,0508,0509,0510,0511,0512,0515,0517,0518,0524$

VII. Protection, Mixtures photons and beta particles $0501,0503,0504,0506,0508,0510,0511,0512,0515,0517,0518,0520,0522,0523,0524$, 0526

VIII. Protection, Mixtures fission neutrons and high energy photons $0501,0504,0509,0510,0512,0515,0517,0518,0522,0524,0526$ 
NBS.114A (REV. 2-8C)

U.S. DEPT. OF COMM.

BIBLIOGRAPHIC DATA

SHEET (See instructions)

1. PUBLICATION OR

REPORT NO.

NBS/SP -687

2. Performing Organ. Report No.

3. Publication Date

February 1985

4. TITLE AND SUBTITLE

1984 NVLAP Directory of Accredited Laboratories

5. AUTHOR(S)

Harvey W. Berger, Editor

6. PERFORMING ORGANIZATION (If joint or other than NBS, see in structions)

7. Contract/Grant No.

NATIONAL BUREAU OF STANDARDS

DEPARTMENT OF COMMERCE.

GAITHERSBURG, MD 20899

8. Type of Report \& Period Covered

Jan.-Dec. 1984

9. SPONSORING ORGANIZATION NAME AND COMPLETE ADDRESS (Street, City. State, ZIP)

Same as in item 6 above.

10. SUPPLEMENTARY NOTES

Library of Congress Catalog Card Number: 84-601165

Document describes a computer program; SF-185, FIPS Software Summary, is attached.

11. ABSTRACT (A 200-word or less factual summary of most significant information. If document includes a significant bibliography or literature survey, mention it here)

Laboratories accredited by the National Voluntary Laboratory Accreditation Program (NVLAP) are identified along with the specific test methods for which they are accredited. The current status of existing accreditation programs is given for laboratories that test thermal insulation, freshly mixed concrete, carpet, wood burning stoves, paint, and personnel radiation dosimeters, and that provide acoustical testing services. Indexes are provided for searching the Directory for laboratories accredited in specific testing areas or for specific test methods.

12. KEY WORDS (Six to twelve entries; clphobetical arder; capitalize only proper names; and seporate key words by semicolons) accreditation; laboratory evaluation; proficiency testing; test methods; testing.

13. AVAILABILITY

$X$ Unlimited

For Official Distribution. Do Not Release to NTIS

Order From Superintendent of Documents, U.S. Government Printing Office, Washington, D.C. 20402.

X Order From National Technical Information Service (NTIS), Springfield, VA. 2216I
14. NO. OF

PRINTED PAGES

79

15. Price 
1 



\section{Technical Publications}

\section{Periodical}

Journal of Research-The Journal of Research of the National Bureau of Standards reports NBS research and development in those disciplines of the physical and engineering sciences in which the Bureau is active. These include physics, chemistry, engineering, mathematics, and computer sciences. Papers cover a broad range of subjects, with major emphasis on measurement methodology and the basic technology underlying standardization. Also included from time to time are survey articles on topics closely related to the Bureau's technical and scientific programs. As a special service to subscribers each issue contains complete citations to all recent Bureau publications in both NBS and non-NBS media. Issued six times a year.

\section{Nonperiodicals}

Monographs-Major contributions to the technical literature on various subjects related to the Bureau's scientific and technical activities.

Handbooks-Recommended codes of engineering and industrial practice (including safety codes) developed in cooperation with interested industries, professional organizations, and regulatory bodies.

Special Publications-Include proceedings of conferences sponsored by NBS, NBS annual reports, and other special publications appropriate to this grouping such as wall charts, pocket cards, and bibliographies.

Applied Mathematics Series-Mathematical tables, manuals, and studies of special interest to physicists, engineers, chemists, biologists, mathematicians, computer programmers, and others engaged in scientific and technical work.

National Standard Reference Data Series-Provides quantitative data on the physical and chemical properties of materials, compiled from the world's literature and critically evaluated. Developed under a worldwide program coordinated by NBS under the authority of the National Standard Data Act (Public Law 90-396).

NOTE: The Journal of Physical and Chemical Reference Data (JPCRD) is published quarterly for NBS by the American Chemical Society (ACS) and the American Institute of Physics (AIP). Subscriptions, reprints, and supplements are available from ACS, 1155 Sixteenth St., NW, Washington, DC 20056.

Building Science Series-Disseminates technical information developed at the Bureau on building materials, components, systems, and whole structures. The series presents research results, test methods, and performance criteria related to the structural and environmental functions and the durability and safety characteristics of building elements and systems.

Technical Notes-Studies or reports which are complete in themselves but restrictive in their treatment of a subject. Analogous to monographs but not so comprehensive in scope or definitive in treatment of the subject area. Often serve as a vehicle for final reports of work performed at NBS under the sponsorship of other government agencies.

Voluntary Product Standards-Developed under procedures published by the Department of Commerce in Part 10, Title 15, of the Code of Federal Regulations. The standards establish nationally recognized requirements for products, and provide all concerned interests with a basis for common understanding of the characteristics of the products. NBS administers this program as a supplement to the activities of the private sector standardizing organizations.

Consumer Information Series-Practical information, based on NBS research and experience, covering areas of interest to the consumer. Easily understandable language and illustrations provide useful background knowledge for shopping in today's technological marketplace.

Order the above NBS publications from: Superintendent of Documents, Government Printing Office, Washington, DC 20402.

Order the following NBS publications-FIPS and NBSIR's-from the National Technical Information Service, Springfield, VA 22161.

Federal Information Processing Standards Publications (FIPS PUB)-Publications in this series collectively constitute the Federal Information Processing Standards Register. The Register serves as the official source of information in the Federal Government regarding standards issued by NBS pursuant to the Federal Property and Administrative Services Act of 1949 as amended, Public Law 89-306 (79 Stat. 1127), and as implemented by Executive Order 11717 (38 FR 12315, dated May 11, 1973) and Part 6 of Title 15 CFR (Code of Federal Regulations).

NBS Interagency Reports (NBSIR)-A special series of interim or final reports on work performed by NBS for outside sponsors (both government and non-government). In general, initial distribution is handled by the sponsor; public distribution is by the National Technical Information Service, Springfield, VA 22161, in paper copy or microfiche form. 
U.S. Department of Commerce National Bureau of Standards

Gaithersburg. MD 20899

Official Business

Penalty for Private Use $\$ 300$
SPECIAL FOURTH-CLASS RATE POSTAGE \& FEES PAID NBS

PERMIT No. G195 